\title{
Dense Gas, Dynamical Equilibrium Pressure, and Star Formation in Nearby Star-forming Galaxies
}

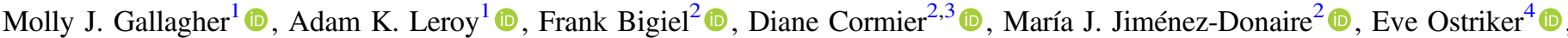 \\ Antonio Usero $^{5}$ (i) , Alberto D. Bolatto ${ }^{6}$ (i), Santiago García-Burillo ${ }^{5}$ (i), Annie Hughes ${ }^{7,8}$ (1), Amanda A. Kepley ${ }^{9}$ (i)

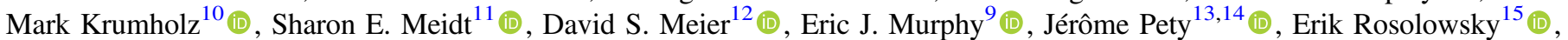 \\ Eva Schinnerer ${ }^{11}$ (iD), Andreas Schruba ${ }^{16}$, and Fabian Walter ${ }^{11}$ (D) \\ ${ }^{1}$ Department of Astronomy, The Ohio State University, 140 West 18th Avenue, Columbus, OH 43210, USA; gallagher.674@osu.edu \\ 2 Institute für theoretische Astrophysik, Zentrum für Astronomie der Universität Heidelberg, Albert-Ueberle Str. 2, D-69120 Heidelberg, Germany \\ ${ }^{3}$ Laboratoire AIM, CEA/DSM-CNRS-Université Paris Diderot, Irfu/Service d'Astrophysique, CEA Saclay, F-91191 Gif-sur-Yvette, France \\ ${ }_{5}^{4}$ Department of Astrophysical Sciences, Princeton University, Princeton, NJ 08544, USA \\ 5 Observatorio Astronómico Nacional (IGN), C/Alfonso XII, 3, E-28014 Madrid, Spain \\ ${ }^{6}$ Department of Astronomy, Laboratory for Millimeter-wave Astronomy, and Joint Space Institute, University of Maryland, College Park, MD 20742, USA \\ CNRS, IRAP, 9 av. du Colonel Roche, BP 44346, F-31028 Toulouse cedex 4, France \\ ${ }^{8}$ Université de Toulouse, UPS-OMP, IRAP, F-31028 Toulouse cedex 4, France \\ ${ }^{9}$ National Radio Astronomy Observatory, 520 Edgemont Road, Charlottesville, VA 22903, USA \\ ${ }^{10}$ Research School of Astronomy \& Astrophysics, Australian National University, Canberra, ACT 2611, Australia \\ ${ }^{11}$ Max Planck Institute für Astronomie, Königstuhl 17, D-69117, Heidelberg, Germany \\ 12 Department of Physics, New Mexico Institute of Mining and Technology, 801 Leroy Place, Soccoro, NM 87801, USA \\ ${ }^{13}$ Institut de Radioastronomie Millimètrique (IRAM), 300 Rue de la Piscine, F-38406 Saint Martin d'Hères, France \\ ${ }^{14}$ Observatoire de Paris, 61 Avenue de l'Observatoire, F-75014 Paris, France \\ 15 Department of Physics, University of Alberta, Edmonton, AB T6G 2E1, Canada \\ ${ }^{16}$ Max-Planck-Institut für extraterrestrische Physik, Giessenbachstraße 1, D-85748 Garching, Germany \\ Received 2017 April 14; revised 2018 March 19; accepted 2018 March 20; published 2018 May 10
}

\begin{abstract}
We use new ALMA observations to investigate the connection between dense gas fraction, star formation rate (SFR), and local environment across the inner region of four local galaxies showing a wide range of molecular gas depletion times. We map $\mathrm{HCN}(1-0), \mathrm{HCO}^{+}(1-0), \mathrm{CS}(2-1),{ }^{13} \mathrm{CO}(1-0)$, and $\mathrm{C}^{18} \mathrm{O}(1-0)$ across the inner few kiloparsecs of each target. We combine these data with short-spacing information from the IRAM large program EMPIRE, archival $\mathrm{CO}$ maps, tracers of stellar structure and recent star formation, and recent $\mathrm{HCN}$ surveys by Bigiel et al. and Usero et al. We test the degree to which changes in the dense gas fraction drive changes in the SFR. $I_{\mathrm{HCN}} / I_{\mathrm{CO}}$ (tracing the dense gas fraction) correlates strongly with $I_{\mathrm{CO}}$ (tracing molecular gas surface density), stellar surface density, and dynamical equilibrium pressure, $P_{\mathrm{DE}}$. Therefore, $I_{\mathrm{HCN}} / I_{\mathrm{CO}}$ becomes very low and $\mathrm{HCN}$ becomes very faint at large galactocentric radii, where ratios as low as $I_{\mathrm{HCN}} / I_{\mathrm{CO}} \sim 0.01$ become common. The apparent ability of dense gas to form stars, $\Sigma_{\mathrm{SFR}} / \Sigma_{\text {dense }}$ (where $\Sigma_{\text {dense }}$ is traced by the HCN intensity and the star formation rate is traced by a combination of $\mathrm{H} \alpha$ and $24 \mu \mathrm{m}$ emission), also depends on environment. $\Sigma_{\mathrm{SFR}} / \Sigma_{\text {dense }}$ decreases in regions of high gas surface density, high stellar surface density, and high $P_{\mathrm{DE}}$. Statistically, these correlations between environment and both $\Sigma_{\mathrm{SFR}} / \Sigma_{\text {dense }}$ and $I_{\mathrm{HCN}} / I_{\mathrm{CO}}$ are stronger than that between apparent dense gas fraction $\left(I_{\mathrm{HCN}} / I_{\mathrm{CO}}\right)$ and the apparent molecular gas star formation efficiency $\Sigma_{\mathrm{SFR}} / \Sigma_{\text {mol }}$. We show that these results are not specific to $\mathrm{HCN}$.
\end{abstract}

Key words: galaxies: ISM - galaxies: star formation - radio lines: ISM

Supporting material: tar.gz file

\section{Introduction}

Observations of star-forming regions in the Milky Way (e.g., Lada \& Lada 2003; Kainulainen et al. 2009; Heiderman et al. 2010; Lada et al. 2010; André et al. 2014) indicate that stars form mainly in dense substructures. These studies have inspired a large body of literature investigating how the amount of dense gas relates to the star formation rate of a cloud or galaxy. Lada et al. (2012) found the star formation rate (SFR) surface density $\left(\Sigma_{\mathrm{SFR}}\right)$ in individual Milky Way clouds to be proportional to the fraction of the gas that is dense (where here "dense" is $\left.n \geqslant 3 \times 10^{4} \mathrm{~cm}^{-3}\right)$. Similarly, both Evans et al. (2014) and Lada et al. (2010) found that the SFR in individual clouds relates linearly to the molecular gas mass above an extinction threshold chosen to select only dense gas: $A_{V} \approx 8 \mathrm{mag}$ and $A_{K} \approx 0.8 \mathrm{mag}$, respectively (see also Könyves et al. 2015). In a pioneering study, Gao \& Solomon (2004) showed that galaxy-integrated SFR, traced by IR emission, relates linearly to the total mass of dense molecular gas, traced by $\mathrm{HCN}$ emission. Based on this result, they argued for a similar picture for whole galaxies, with the SFR set by the amount of dense, $\mathrm{HCN}$-emitting gas.

Spectroscopic studies of other galaxies indicate a more complex relationship between dense gas and SFR. Usero et al. (2015) surveyed $\sim 60$ regions across 30 star-forming galaxies with a $\sim 1-2 \mathrm{kpc}$ sized beam. They found that the dense gas star formation efficiency $\left(\mathrm{SFE}_{\text {dense }}\right.$, defined as $\left.\Sigma_{\mathrm{SFR}} / \Sigma_{\text {dense }}\right)$, inferred from $\mathrm{HCN}, \mathrm{H} \alpha$, and $24 \mu \mathrm{m}$ emission, anticorrelates with both the stellar surface density $\left(\Sigma_{*}\right)$ and the fraction of gas in the molecular phase $\left(f_{\mathrm{mol}}\right)$. Using one of the first wholegalaxy HCN galaxy maps, Bigiel et al. (2016) found similar trends across the disk of NGC 5194 (consistent with Chen et al. 2015): at roughly kiloparsec resolution, $\mathrm{SFE}_{\text {dense }}$ drops with increasing $\Sigma_{*}$ and increasing $f_{\mathrm{mol}}$. The sense of these results agrees with recent work studying the Milky Way, which finds the rate of star formation per unit dense gas to be lower in 
the Galactic center than in solar neighborhood molecular clouds (see Longmore et al. 2013).

The abundance of dense gas is also of interest and appears to depend strongly on environment. Gao \& Solomon (2004) observed large variations in the dense gas fraction $\left(f_{\text {dense }} \equiv\right.$ $\Sigma_{\text {dense }} / \Sigma_{\text {mol }}$ ), traced by the $I_{\mathrm{HCN}} / I_{\mathrm{CO}}$ ratio. Usero et al. (2015) and Bigiel et al. (2016) found that $f_{\text {dense }}$ varies significantly, correlating with $\Sigma_{*}$ and $f_{\text {mol }}$. Furthermore, the Milky Way center appears far richer in dense gas (Longmore et al. 2013) than the local clouds studied by Lada et al. (2010) and Evans et al. (2014).

Usero et al. (2015) and Bigiel et al. (2016) found that $\mathrm{SFE}_{\text {dense }}$ and $f_{\text {dense }}$ vary as a function of $\Sigma_{*}$ and $f_{\mathrm{mol}}$. Both $\Sigma_{*}$ and $f_{\text {mol }}$ relate closely to the interstellar pressure needed to support a gas disk in vertical dynamic equilibrium, $P_{\mathrm{DE}}$ (Elmegreen 1989). We know that $P_{\mathrm{DE}}$ correlates closely with $f_{\text {mol }}$ (Wong \& Blitz 2002; Blitz \& Rosolowsky 2006; Leroy et al. 2008) and with the internal pressure of molecular clouds (Hughes et al. 2013). We also expect a correlation between $P_{\mathrm{DE}}$ and the density of the interstellar gas. Indeed, Helfer \& Blitz (1997) suggested this correlation to help explain the paucity of HCN emission outside galaxy centers.

This central role of gas pressure and interstellar medium (ISM) weight appears to be consistent with star formation selfregulation theory (see e.g., Ostriker et al. 2010; Kim et al. 2011; Ostriker \& Shetty 2011) and simulations (see e.g., Kim et al. 2011, 2013; Kim \& Ostriker 2015), which predict that $\Sigma_{\mathrm{SFR}}$ will be proportional to the total pressure. However, $P_{\mathrm{DE}}$ should not be the only factor at play. The turbulent Mach number (e.g., Krumholz \& Thompson 2007; García-Burillo et al. 2012; Usero et al. 2015), virial parameter (Kruijssen et al. 2014), and large-scale kinematics (Meidt et al. 2018) may also influence $\mathrm{SFE}_{\mathrm{dense}}$.

Testing these ideas and advancing this field requires more resolved multiline mapping of galaxies, in the style of Chen et al. (2015), Usero et al. (2015), and Bigiel et al. (2016). In this approach, one observes a suite of lines whose emissivity peaks at different densities, $n_{\text {eff }}{ }^{17}$ To first order, molecular line emission will be proportional to the mass of gas above $n_{\mathrm{eff}}$, as long as all other physical conditions remain fixed. Thus, changes in the ratio of intensities between two emission lines with different $n_{\text {eff }}$ can indicate a changing ratio of masses above each density (though there are crucial subtleties; see, e.g., Krumholz \& Thompson 2007; Leroy et al. 2017b). Because this method gives access to changes in the density distribution without the need to resolve molecular cloud substructure, it can be deployed to study changing gas density distributions across whole galaxies or large parts of galaxies. This, in turn, gives access to a much wider range of physical conditions in which we can study the origin and role of dense gas.

Until recently, interferometers have lacked the surface brightness sensitivity to survey high- $n_{\text {eff }}$ lines like HCN (1-0) across the disks of nearby normal galaxies (for an early attempt limited by sensitivity, see Helfer \& Blitz 1997). HCN (1-0), often the brightest dense gas tracer, can be $\sim 30$ or more times fainter than CO (1-0) (Usero et al. 2015). The Atacama Large Millimeter/ submillimeter Array (ALMA) changes this. ALMA makes it practical to map entire nearby galaxies with multiline, densitysensitive spectroscopy. The high sensitivity of ALMA allows us

\footnotetext{
$17 n_{\text {eff }}$ refers to the lowest density for which a line achieves $95 \%$ of its maximum emissivity at a given $T_{\mathrm{kin}}$ and $\tau$. It is closely related, though not identical, to the effective critical density; see Shirley (2015) and Leroy et al. (2017b).
}

to reach noise levels comparable to those of previous single-dish maps (e.g., Bigiel et al. 2016) in less than an hour. The high resolution of ALMA helps to bridge the gap in scale between the individual molecular clouds and cores studied in the Milky Way and galaxy averages used in previous seminal studies (e.g., Gao \& Solomon 2004; García-Burillo et al. 2012). ALMA's resolution also makes it possible to distinguish distinct dynamical regions, for example, disentangling the nuclear gas structures in galaxies (analogs to the Milky Way's "central molecular zone," CMZ) from extended emission associated with the disk and spiral arms.

Here, we employ ALMA multiline spectroscopy to study the origin and role of dense molecular gas in nearby galaxies. Our main goals are to measure any variations in the dense gas fraction and the star formation efficiency of dense gas and to understand the physical drivers of such variations. We present new ALMA observations of NGC 3351, NGC 3627, NGC 4254, and NGC 4321. These observations cover high- $n_{\text {eff }}$ transitions ( $\mathrm{HCN}(1-0), \mathrm{HCO}^{+}(1-0)$, and $\left.\mathrm{CS}(2-1)\right)$ and two $\mathrm{CO}$ isotopologues $\left({ }^{13} \mathrm{CO}(1-0)\right.$ and $\left.\mathrm{C}^{18} \mathrm{O}(1-0)\right)$ across the inner $\approx 1^{\prime}(\approx 3-5 \mathrm{kpc})$ of each galaxy. We chose these four targets because together they show a wide range of apparent SFR per unit molecular gas $\left(\mathrm{SFE}_{\mathrm{mol}}\right)$ over their inner few kiloparsec (Leroy et al. 2013b). By observing the dense gas and contrasting it with SFR tracers and CO imaging, we aim to understand if these apparent variations in the $\mathrm{SFE}_{\text {mol }}$ within and among our targets are driven by changes in the dense gas fraction. We also aim to understand the physical drivers of the dense gas fraction.

In this paper, we combine these new observations with the data from Usero et al. (2015) and Bigiel et al. (2016) to test the hypotheses that (1) the dense gas fraction alone drives the SFR and (2) the mean midplane pressure drives the density distribution and the role of dense gas in star formation.

In Section 2, we describe our ALMA observations (Section 2.1), previous CO observations (Section 2.2), and data processing (Section 2.4). We also summarize the multiwavelength data used in our analysis (Sections 2.5-2.8). Then, in Section 3, we describe some qualitative properties of our data (Section 3.1) and explore the quantitative relationship between dense gas and SFR in our sample (Section 3.2). We discuss the validity of using $I_{\mathrm{HCN}} / I_{\mathrm{CO}}$ as a tracer of gas density (Section 3.3 ) and then explore what sets the star formation efficiency of dense gas (Section 3.4) and the dense gas fraction (Section 3.5), highlighting the possible role of ISM pressure (Section 3.6). In Section 4 we discuss the implications of our results, including theoretical implications for the link between gas density, star formation, and galactic environment (Section 4.1). Finally, we lay out key caveats and next steps (Section 4.2), and then we summarize our findings in Section 5.

\section{Data}

We mapped tracers of dense gas over the inner regions of NGC 3351, NGC 3627, NGC 4254, and NGC 4321. We chose these targets from the sample of nearby galaxies of Leroy et al. (2013b), which had the best available supporting multiwavelength data (CO, $\mathrm{HI}$, infrared (IR), $\mathrm{H} \alpha$, etc., mapping) at the time of the proposal. Based on the measured IR surface brightness of the targets in that study, we estimated the likely HCN emission of each target (following Gao \& Solomon 2004; Usero et al. 2015). Out of the subset of Leroy et al. (2013b) targets that could feasibly be detected by ALMA, we chose these four because together they spanned a large range of $\mathrm{CO}$ to- $24 \mu \mathrm{m}$ ratios over their inner few kiloparsecs (see plots 
Table 1

Lines Observed

\begin{tabular}{lccc}
\hline \hline Line & $\begin{array}{c}\nu_{\text {rest }}^{\mathrm{a}} \\
(\mathrm{GHz})\end{array}$ & Fiducial $\tau^{\mathrm{b}}$ & $\begin{array}{c}n_{\text {eff }}^{\mathrm{c}} \\
\left(\mathrm{cm}^{-3}\right)\end{array}$ \\
\hline${ }^{12} \mathrm{CO}(1-0)$ & 115.27 & 10 & $1 \times 10^{2}$ \\
${ }^{13} \mathrm{CO}(1-0)$ & 110.20 & 0.1 & $8 \times 10^{2}$ \\
$\mathrm{C}^{18} \mathrm{O}(1-0)^{\mathrm{d}}$ & 109.78 & 0.1 & $8 \times 10^{2}$ \\
$\left.\mathrm{CS}^{2-1}\right)^{\mathrm{d}}$ & 97.98 & 1 & $7 \times 10^{4}$ \\
$\mathrm{HCO}^{+}(1-0)$ & 89.19 & 1 & $4 \times 10^{4}$ \\
$\mathrm{HCN}(1-0)$ & 88.63 & 1 & $2 \times 10^{5}$ \\
\hline
\end{tabular}

Notes.

${ }^{a}$ From Splatalogue (http://splatalogue.net/).

b Typical optical depth. See Jiménez-Donaire et al. (2017a, 2017b) for more details.

${ }^{\mathrm{c}}$ Density at which the emissivity reaches $95 \%$ of its maximum given our fiducial $\tau$ and taking $T_{\text {kin }}=25 \mathrm{~K}$. From Leroy et al. (2017b).

${ }^{\mathrm{d}}$ CS (2-1) was only observed by ALMA, not the IRAM $30 \mathrm{~m}$, and so not covered in NGC 5194 and not short-spacing corrected in the other targets.

below). This implies variations in the efficiency with which the total molecular gas reservoir forms stars, making this small sample ideal to test the hypothesis that variations in the molecular gas depletion time are driven by variations in the dense gas fraction.

\subsection{ALMA Molecular Line Observations}

As part of ALMA's Cycle 2 campaign, we observed $\mathrm{HCN}(1-0), \quad \mathrm{HCO}^{+}(1-0), \quad \mathrm{CS}(2-1),{ }^{13} \mathrm{CO}(1-0)$, and $\mathrm{C}^{18} \mathrm{O}(1-0)$ in four galaxies using ALMA's main array of $12 \mathrm{~m}$ antennas. For the remainder of the paper, we will refer to these lines as $\mathrm{HCN}, \mathrm{HCO}^{+}, \mathrm{CS},{ }^{13} \mathrm{CO}$, and $\mathrm{C}^{18} \mathrm{O} . \mathrm{HCN}$, $\mathrm{HCO}^{+}$, and $\mathrm{CS}$ all have $n_{\mathrm{eff}} \sim 10^{4}-10^{5} \mathrm{~cm}^{-3}$ (see Table 1) and so are expected to trace mainly dense gas (though in the absence of such gas they can still emit; e.g., Shirley 2015; Leroy et al. 2017b). The $\mathrm{CO}$ isotopologues, ${ }^{13} \mathrm{CO}$ and $\mathrm{C}^{18} \mathrm{O}$, trace lower-density gas, $n_{\text {eff }} \sim 10^{3} \mathrm{~cm}^{-3}$. The contrast between the optically thin isotopologues and the optically thick ${ }^{12} \mathrm{CO}$ emission constrains the optical depth and physical conditions in the bulk of the molecular gas. We make limited use of ${ }^{13} \mathrm{CO}$ and $\mathrm{C}^{18} \mathrm{O}$ in this paper. These data are analyzed in detail by Jiménez-Donaire et al. (2017a). Fainter lines in the bandpass were analyzed by Jiménez-Donaire et al. (2017b).

Table 2 gives our adopted position, morphology, orientation, distance, beam size, and field of view for each target. We observed seven fields in a hexagonally packed mosaic pointed toward the center of each galaxy. The mosaic pattern used the default Nyquist spacing set by the ALMA observing tool.

We observed each galaxy with two spectral setups. The first covered lines tracing the dense gas: $\mathrm{HCN}, \mathrm{HCO}^{+}$, and $\mathrm{CS}$. The four spectral windows covered $85.4-87.2 \mathrm{GHz}, 87.2$ 89.0 GHz, 97.2-99.0 GHz, and 99.0-100.8 GHz. The second spectral setup covered lines tracing the overall distribution of molecular gas: ${ }^{13} \mathrm{CO}$ and $\mathrm{C}^{18} \mathrm{O}$. Those four spectral windows covered $98.2-100.0 \mathrm{GHz}, \quad 96.6-98.4 \mathrm{GHz}, \quad 108.5-110.3 \mathrm{GHz}$, and $110.3-112.1 \mathrm{GHz}$. For both setups, we observed using a channel width of $976.6 \mathrm{kHz}\left(\sim 3 \mathrm{~km} \mathrm{~s}^{-1}\right.$ at $\left.\nu=100 \mathrm{GHz}\right)$ and bandwidth of $1.875 \mathrm{GHz}$, sufficient to cover the full velocity extent of each line in question.

We observed in a compact configuration in order to emphasize flux recovery and surface brightness sensitivity, reflecting the faint nature of the dense gas tracers. After calibration, the HCN observations had 703 (NGC 3351), 630 (NGC 3627), 561 (NGC 4254), and 561 (NGC 4321) baselines, with minimum and maximum unprojected lengths of 15 and $348 \mathrm{~m}$, respectively, median baseline length of 90-100 m, and $20 \%-90 \%$ range of typically $50-195 \mathrm{~m}$. For reference at the $\nu \sim 89.5 \mathrm{GHz}$ of the $\mathrm{HCN}$ and $\mathrm{HCO}^{+}$lines, 50, 100, and $200 \mathrm{~m}$ correspond to $\sim 13 . " 8,6 . " 9$, and $3 . " 5$.

We processed the data using the the CASA software package (McMullin et al. 2007) and the observatory-provided calibration scripts. Most of the calibration occurred in CASA version 4.2.2, with one data set calibrated in CASA version 4.3.1. The calibration scripts were a mixture of the observatory-produced CASA scripts and calls to the formal ALMA pipeline. In all cases they are available, along with the data, from the ALMA archive. After inspecting the pipeline calibrated data, we imaged each line separately. For the final version of the imaging, we used CASA version 4.6.0.

We first subtracted continuum emission using the CASA task uvcontsub and avoiding the frequencies of known bright lines. We then imaged each cube using natural weighting, averaging in frequency to produce $10 \mathrm{~km} \mathrm{~s}^{-1}$ wide channels, and applying a small $u-v$ taper $\left(2^{\prime \prime}-3^{\prime \prime}\right.$ depending on the line and target). The taper further emphasizes surface brightness sensitivity. The small loss of resolution is irrelevant to the science in this paper because the comparison to tracers of recent star formation already limits our work to $\gtrsim 5^{\prime \prime}$ resolution.

With the taper, but before any other processing, the synthesized beams in the deconvolved HCN images were

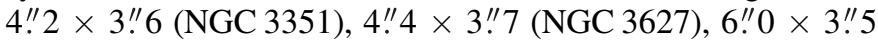
(NGC 4254), and 4!" $6 \times 3$ !" 2 (NGC 4321). The pixel size adopted during imaging was always chosen to heavily oversample the beam. After imaging, we convolved each cube using the CASA task imsmooth to have a round $8^{\prime \prime} \times 8^{\prime \prime}$ Gaussian beam. This allowed us to beam-match the poorerresolution $\mathrm{CO}$ and infrared data that are crucial to the analysis. The final images used in this analysis all have $8^{\prime \prime}$ (FWHM) beams, $10 \mathrm{~km} \mathrm{~s}^{-1}$ channel width, and 0 .'5 pixels that heavily oversample the beam.

Given the $u-v$ coverage mentioned above, we expect structures larger than $\sim 14^{\prime \prime}$ in a single channel to suffer from spatial filtering in the ALMA main array data. To account for this, we combined our $12 \mathrm{~m} \mathrm{HCN}, \mathrm{HCO}^{+},{ }^{13} \mathrm{CO}$, and $\mathrm{C}^{18} \mathrm{O}$ data with single-dish maps obtained as part of the IRAM EMPIRE Survey (Bigiel et al. 2016; Cormier et al. 2018, M. Jimenez Donaire et al. 2018, in preparation). To do this, we aligned the IRAM maps to the grid of the ALMA data, applied the primary beam response of the ALMA images to the IRAM data, and converted the IRAM data to have units of Jy beam ${ }^{-1}$. Then we combined the two data sets using the CASA task feather. After the combination, we verified that the feathered cube indeed matched the spectral profile of the IRAM 30 m cube when both were convolved to a common $30^{\prime \prime}$ resolution. Short-spacing data were not available for the CS, so those data are from ALMA's main $12 \mathrm{~m}$ array only in this paper.

Table 3 reports the total flux recovered for each line from each galaxy both with and without the addition of the IRAM $30 \mathrm{~m}$ data. We calculate the total flux by summing the pixels in the original data cubes. The table shows that ALMA recovers $\gtrsim 95 \%$ of the flux for all lines in NGC 3351, which is 
Table 2

Targets Observed

\begin{tabular}{|c|c|c|c|c|c|c|c|c|}
\hline Galaxy & $\begin{array}{l}\text { R.A. } \\
\text { (J2000) }\end{array}$ & $\begin{array}{l}\text { Decl. } \\
(\mathrm{J} 2000)\end{array}$ & Morphology & $\begin{array}{c}\text { Inclination } \\
\text { (degree) }\end{array}$ & $\begin{array}{l}\text { Position Angle } \\
\quad \text { (degree) }\end{array}$ & $\begin{array}{l}\text { Distance } \\
(\mathrm{Mpc})\end{array}$ & $\begin{array}{l}\text { Linear Beam } \\
(\mathrm{pc})\end{array}$ & $\begin{array}{l}\text { FOV } \\
(\mathrm{kpc})\end{array}$ \\
\hline NGC 3351 & 160.990417 & 11.703806 & $\mathrm{SB}(\mathrm{r}) \mathrm{b}$ & 41.0 & 192.0 & 9.33 & 392 & 3.67 \\
\hline NGC 3627 & 170.0623508 & 12.9915378 & $\mathrm{SAB}(\mathrm{s}) \mathrm{b}$ & 62.0 & 173.0 & 9.38 & 380 & 3.56 \\
\hline NGC 4321 & 185.728463 & 15.821818 & $\mathrm{SAB}(\mathrm{s}) \mathrm{bc}$ & 30.0 & 153.0 & 14.3 & 636 & 5.96 \\
\hline NGC $5194^{30 m}$ & 202.4667 & 47.1947 & $\mathrm{SA}(\mathrm{s}) \mathrm{bc}$ pec & 22.0 & 7.5 & 7.6 & 295 & $9.22 \times 12.55$ \\
\hline
\end{tabular}

Note. R.A., decl.: adopted center of the galaxy. Inclination: inclination used to construct the radial profiles and correct surface densities for projection. Position Angle: position angle measured north through east used to construct the radial profiles. Distance: adopted distance to the galaxy in Mpc. Linear Beam: linear scale in pc of the FWHM of the beam used in this analysis. This is $8^{\prime \prime}$ at the distance of the galaxy for the first four targets and $30^{\prime \prime}=1.1 \mathrm{kpc}$ for NGC 5194 (Bigiel et al. 2016 ). FOV: field of view across the dense gas maps at the distance of the galaxy without accounting for inclination. References: centers and morphologies adopted from the NASA Extragalactic Database, which draws key information from RC3 (de Vaucouleurs et al. 1991). Source for orientation parameters: NGC 3351, Dicaire et al. (2008); NGC 3627, de Blok et al. (2008); NGC 4254, Boissier et al. (2003); NGC 4321, Muñoz-Mateos et al. (2009); NGC 5194, Colombo et al. (2014). Distances adopted from Kennicutt et al. (2011).

${ }^{30 \mathrm{~m}}$ Observed with the IRAM $30 \mathrm{~m}$. Data from Bigiel et al. (2016); see that paper for more details. The other targets were all observed with ALMA with short- and zero-spacing correction from the IRAM $30 \mathrm{~m}$. See Section 2.

dominated by a bright, compact nuclear source. In fact, for the two faintest lines, ALMA finds slightly more flux than the IRAM $30 \mathrm{~m}$, indicating modest $(\sim 10 \%)$ discrepancies in the flux calibration scale. ALMA recovers a lower fraction of the flux for galaxies with more extended, low signal-to-noise ratio $(\mathrm{S} / \mathrm{N})$ emission. On average, ALMA recovers $\sim 60 \%-$ $80 \%$ of the flux found by the IRAM $30 \mathrm{~m}$. Our worst case is NGC 4254, where the faint line emission appears extended and ALMA recovers only $\sim 30 \%-50 \%$ of the flux seen by the IRAM $30 \mathrm{~m}$. This likely results from two factors: the large extent of the $\mathrm{CO}$ emission in the galaxy and the lack of a compact, bright nuclear source (in contrast to the other three targets). This variable, sometimes poor flux recovery emphasizes the importance of including short-spacing data.

Throughout the paper, we work with intensity in units of brightness temperature, $T_{B}$. We convert our final data cubes from their native units of $\mathrm{Jy}_{\text {beam }}{ }^{-1}$ to $T_{B}$ via the standard Rayleigh-Jeans formula:

$$
T_{B}[\mathrm{~K}]=\frac{c^{2}}{2 \times 10^{23} k_{B} \nu^{2}} I_{\nu}[\mathrm{Jy} / \text { beam }],
$$

where $\nu$ is the frequency of the line, $k_{B}$ is Boltzmann's constant, and $I_{\nu}$ is the original intensity in $\mathrm{Jy} \mathrm{beam}^{-1}$.

Finally, we measure the rms noise from the signal-free region of each cube. At $8^{\prime \prime}$ resolution, the statistical noise in each $10 \mathrm{~km} \mathrm{~s}^{-1}$ channel is $\sim 5-10 \mathrm{mK}$ for $\mathrm{HCN}, \mathrm{HCO}^{+}$, and $\mathrm{CS}$ and $\sim 10 \mathrm{mK}$ for ${ }^{13} \mathrm{CO}$ and $\mathrm{C}^{18} \mathrm{O}$. These vary somewhat from cube to cube, and we use the correct local value to construct uncertainty maps for the integrated intensity. The nominal flux calibration accuracy of ALMA in Band 3 during Cycle 2 was $5 \%$ according to the ALMA Cycle 2 Technical Handbook, though this may be somewhat optimistic. The IRAM $30 \mathrm{~m}$ intensity calibration for EMPIRE observations is internally consistent to $\approx 5 \%$ from night to night, but the absolute calibrations scale is uncertain at the $10 \%-15 \%$ level (M. Jimenez Donaire et al. 2018, in preparation).

\subsection{CO Observations}

We use ${ }^{12} \mathrm{CO}(1-0)$ emission, hereafter referred to as $\mathrm{CO}$, to trace the overall molecular gas reservoir. We draw these maps from literature data. In each case, our CO data include both interferometric and single-dish data, allowing us to reach our
Table 3

Flux Recovery

\begin{tabular}{lcccc}
\hline \hline Data Type & $\begin{array}{l}{ }^{13} \mathrm{CO} \\
\left(10^{3} \mathrm{~K} \mathrm{~km} \mathrm{~s}^{-1} \mathrm{Crcsec}^{2}\right)\end{array}$ & $\mathrm{HCO}^{+}$ & $\mathrm{HCN}$ \\
\hline NGC 3351 & & & & \\
ALMA+IRAM 30 m & 3.32 & 0.41 & 1.21 & 2.24 \\
ALMA only & 3.20 & 0.45 & 1.24 & 2.14 \\
Fraction recovered & 0.96 & 1.11 & 1.02 & 0.96 \\
NGC 3627 & & & & \\
ALMA+IRAM 30 m & 16.98 & 1.55 & 4.96 & 6.47 \\
ALMA only & 11.98 & 1.40 & 3.77 & 4.39 \\
Fraction recovered & 0.71 & 0.91 & 0.76 & 0.68 \\
NGC 4254 & & & & \\
ALMA+IRAM 30 m & 15.98 & 3.31 & 3.98 & 4.34 \\
ALMA only & 6.84 & 0.67 & 1.11 & 1.42 \\
Fraction recovered & 0.43 & 0.20 & 0.28 & 0.33 \\
NGC 4321 & & & & \\
ALMA+IRAM 30 m & 14.52 & 2.61 & 3.86 & 5.10 \\
ALMA only & 8.79 & 1.81 & 2.20 & 3.55 \\
Fraction recovered & 0.61 & 0.70 & 0.57 & 0.70 \\
\hline
\end{tabular}

working $\sim 8^{\prime \prime}$ resolution, but also recover zero- and shortspacing information. All of the $\mathrm{CO}$ data have a larger field of view than our ALMA maps, bandwidth that covers the entire $\mathrm{CO}$ line for the galaxy, and pixels that oversample the beam by more than the Nyquist rate.

For NGC 3351 and NGC 3627, we use CO observations from BIMA SONG (Helfer et al. 2003). These cubes include data from both the BIMA interferometer and the NRAO $12 \mathrm{~m}$ single-dish telescope on Kitt Peak. The combined BIMA+12 m cubes have native resolutions of 7 !! $4 \times 5$ !! $2 \times 10 \mathrm{~km} \mathrm{~s}^{-1}$ (NGC 3351) and 7!"3 $\times 5 . ! 8 \times 10 \mathrm{~km} \mathrm{~s}^{-1}$ (NGC 3627). Helfer et al. (2003) quote a flux calibration uncertainty of $15 \%$.

For NGC 4254, we use interferometric CO observations from CARMA STING (Rahman et al. 2011) and single-dish data from the $\mathrm{CO}$ extension to the IRAM EMPIRE survey (Cormier et al. 2018, M. Jiménez-Donaire et al. in preparation). Before convolution, the native resolution of the CARMA data is 3 !" $3 \times 2$ ". $7 \times 5 \mathrm{~km} \mathrm{~s}^{-1}$. Rahman et al. (2011) do not quote an amplitude calibration uncertainty but discuss $\sim 10 \%$ as a typical value. We combine the CARMA and IRAM data using 
the CASA task feather, which carries out a Fourier plane combination of the two cubes.

For NGC 4321, we use CO data provided as part of the ALMA science verification program. These include both main $12 \mathrm{~m}$ array and Atacama Compact Array short-spacing and total power data. We use the feathered "reference image" provided as part of the science verification release, which has resolution 3 !" $9 \times 2$ ". $5 \times 5 \mathrm{~km} \mathrm{~s}^{-1}$. As above, the nominal amplitude calibration accuracy for ALMA at Band 3 is $\pm 5 \%$, though in practice this seems optimistic. We convert all $\mathrm{CO}$ data to units of brightness temperature following Equation (1), convolve them to our working $8^{\prime \prime}$ resolution, and align them to the astrometric and velocity grid of the ALMA dense gas data.

\subsection{CO and Dense Gas Conversion Factors}

Whenever possible, we report our results in terms of observable quantities, e.g., $I_{\mathrm{CO}}, I_{\mathrm{HCN}}$, etc. We also express our results in terms of physical quantities, e.g., molecular gas mass and dense gas mass. The translation from observed to physical quantities carries substantial uncertainty and remains a topic of active research (e.g., Sandstrom et al. 2013; Usero et al. 2015; Leroy et al. 2017b). However, these physical quantities-e.g., $\Sigma_{\mathrm{H} 2}$ and $\Sigma_{\text {dense }}$-are of considerable interest and are central to our science goals. Therefore, following Usero et al. (2015), we will also express our results as best-estimate physical terms. Given a choice, we prefer the simplest possible translation from observed to physical quantities and will plot both axes whenever we can.

By default, we quote total molecular gas mass assuming a $\mathrm{CO}(1-0)$ to molecular gas mass conversion factor of $\alpha_{\mathrm{CO}} \approx 4.3 M_{\odot} \mathrm{pc}^{-2}\left(\mathrm{~K} \mathrm{~km} \mathrm{~s}^{-1}\right)^{-1}$. In order to derive a fiducial dense gas mass, we convert from $\mathrm{HCN}$ intensity to dense gas mass surface density assuming $\alpha_{\mathrm{HCN}} \approx 10 M_{\odot} \mathrm{pc}^{-2}\left(\mathrm{~K} \mathrm{~km} \mathrm{~s}^{-1}\right)^{-1}$. Both factors include helium.

Both of these conversion factors carry substantial uncertainty, though for different reasons. Our $\alpha_{\mathrm{CO}} \approx$ $4.3 M_{\odot} \mathrm{pc}^{-2}\left(\mathrm{~K} \mathrm{~km} \mathrm{~s}^{-1}\right)^{-1}$ is often taken as the Milky Way conversion factor and applied as a default to solar-metallicity massive galaxies (see Bolatto et al. 2013). However, dust-based studies that include our current targets suggest that the gas-rich regions in the central parts of some galaxies have lower $\alpha_{\mathrm{CO}}$ (Sandstrom et al. 2013). This presumably reflects dynamical broadening of the $\mathrm{CO}$ line width, leading to more $\mathrm{CO}$ emission per unit mass.

Meanwhile, we consider $\alpha_{\mathrm{HCN}}$ uncertain because the abundance and opacity of $\mathrm{HCN}$ as a function of density and environment remain poorly known. Gao \& Solomon (2004) argue for $\alpha_{\mathrm{HCN}} \approx 10 M_{\odot} \mathrm{pc}^{-2}\left(\mathrm{~K} \mathrm{~km} \mathrm{~s}^{-1}\right)^{-1}$ to convert from $\mathrm{HCN}$ intensity to surface density of gas above $n_{\mathrm{H} 2} \sim 3 \times 10^{4}$ $\mathrm{cm}^{-3}$ based on large velocity gradient modeling and invoking the virial theorem. However, if the inputs to these calculations, e.g., the abundance of $\mathrm{HCN}$, the opacity of $\mathrm{HCN}$, or the dynamical state of dense gas, vary from their assumed values, then the absolute value of $\alpha_{\mathrm{HCN}}$ also changes (see, e.g., Leroy et al. 2017b). Constraints on these quantities in other galaxies remain very weak (see Martín et al. 2006; Jiménez-Donaire et al. 2017a), but observations of the Milky Way do demonstrate important variations (e.g., Pety et al. 2017).

In this paper, we focus on the dense gas fraction. Many environmental factors that affect $\mathrm{CO}$ might also affect $\mathrm{HCN}$ emission. Because we lack a useful prescription for $\alpha_{\mathrm{HCN}}$, it is unclear how we should implement variations in $\alpha_{\mathrm{CO}}$ (for an in-depth discussion of how these quantities may relate, see Usero et al. 2015). Moreover, we aim to clearly present our results in terms of observed line ratios, e.g., $I_{\mathrm{HCN}} / I_{\mathrm{CO}}$. This requires a simple $\alpha_{\mathrm{CO}}$ and $\alpha_{\mathrm{HCN}}$.

We do consider how our multiline observations support the idea that $\mathrm{HCN}$ traces the dense gas. For a complete discussion of the variation in $\alpha_{\mathrm{CO}}, \alpha_{\mathrm{HCN}}$, and their effects on $f_{\text {dense }}$ and $\mathrm{SFE}_{\text {dense, }}$ we refer the reader to Usero et al. (2015).

\subsection{Creation of Integrated Intensity Maps}

For each galaxy, we create a position-position-velocity mask using the $\mathrm{CO}$ data. We first identify pixels with $\mathrm{S} / \mathrm{N}>5$ in at least two adjacent velocity channels. We then remove contiguous $\mathrm{S} / \mathrm{N}>5$ regions that are small compared to the size of the beam. Next, we grow these regions to include adjoining pixels where $\mathrm{S} / \mathrm{N}>2$. The resulting mask does a good job of capturing the region of bright $\mathrm{CO}$ emission that one would identify by eye.

$\mathrm{CO}$ emission tends to be brighter and easier to excite than emission from rarer isotopologues or dense gas tracers. Therefore, we take this region of bright $\mathrm{CO}$ emission to also represent the position-position-velocity region where we might find these fainter lines. We verify by eye that no clear dense gas tracer emission extends beyond this mask. Thus, regions outside this mask are taken to be signal free and used to estimate the rms noise in each cube.

We sum the masked line data cubes along each line of sight to produce maps of integrated intensity, in $\mathrm{K} \mathrm{km} \mathrm{s}^{-1}$. In this sum, masked pixels have a value of zero, and lines of sight with no identified signal have an integrated intensity of zero. Thus, we also produce a $2 \mathrm{D}$ mask indicating which lines of sight include any bright signal in the $\mathrm{CO}$ cube.

We calculate the statistical uncertainty in the integrated intensity from the rms noise in an individual channel map (in $\mathrm{K}$ ) times the channel width (in $\mathrm{km} \mathrm{s}^{-1}$ ) times the square root of unmasked voxels along a line of sight. Typical rms uncertainties due to statistical errors in the integrated lines are $\sim 0.2 \mathrm{~K} \mathrm{~km} \mathrm{~s}^{-1}$ for the $\mathrm{CO}$ isotopologues and $\sim 0.1 \mathrm{~K} \mathrm{~km} \mathrm{~s}^{-1}$ for the dense gas tracers. The $\mathrm{CO}$ data tend to have poorer sensitivity, with typical uncertainty $\sim 2 \mathrm{~K} \mathrm{~km} \mathrm{~s}^{-1}$ in the integrated intensity per line of sight. However, because the $\mathrm{CO}$ is brighter, the $\mathrm{CO}$ data still have higher $\mathrm{S} / \mathrm{N}$.

\subsection{Star Formation Rates}

The deep multiwavelength data available for our sample allow the prospect to estimate the surface density of recent star formation, $\Sigma_{\mathrm{SFR}}$, in several ways. In our table of radial profiles, we provide the measurements necessary to reconstruct most popular monochromatic or "hybrid" tracers (see, e.g., review in Kennicutt \& Evans 2012).

In the plots accompanying the main text, we present $\Sigma_{\mathrm{SFR}}$ estimated from a combination of $\mathrm{H} \alpha$ and $24 \mu \mathrm{m}$ emission following Calzetti et al. (2007) and Leroy et al. (2012). We adopt this choice because for nearby nonstarburst galaxies it has emerged as a widely accepted estimator of $\Sigma_{\text {SFR }}$ (see Kennicutt \& Evans 2012).

Although $\mathrm{H} \alpha+24 \mu \mathrm{m}$ is widely accepted, its calibration remains fundamentally empirical, with limited fundamental work on resolved targets (Calzetti et al. 2007; Kennicutt et al. 2007; Murphy et al. 2011). Much of the literature surrounding dense gas remains focused on infrared luminosity, considering 
the estimated bolometric luminosity (reprocessed by dust) of the young stellar population a more physical tracer of recent star formation activity. Therefore, as a complement to the $\mathrm{H} \alpha$ $+24 \mu \mathrm{m}$ estimates shown in the main plots, appendix investigates estimates of the total infrared (TIR) surface brightness. We convolve multiband Herschel and Spitzer imaging to a common, coarse resolution and apply the prescriptions of Galametz et al. (2013) for each galaxy. Based on this, we calibrate galaxy-by-galaxy conversions from the higher-resolution $70 \mu \mathrm{m}$ data to $\Sigma_{\mathrm{TIR}}$. These data are provided in the table of radial profiles and can be used to carry out a parallel analysis to the one presented in the main text.

The numerical results from these two main approaches to $\Sigma_{\text {SFR }}$ differ at the $\sim 30 \%$ level. However, the choice of SFR tracer does not affect our qualitative conclusions. For either case, dense gas fraction appears to be a poor predictor of the bulk star formation efficiency, and the SFR per unit dense gas drops in the high surface density, inner parts of several of our targets.

$$
\text { 2.5.1. } H \alpha+24 \mu m
$$

We calculate the surface density of recent star formation using the prescription of Calzetti et al. (2007) recast into surface brightness units. Their prescription (their Equation (7)) in luminosity units is

$$
\operatorname{SFR}\left[M_{\odot} \mathrm{yr}^{-1}\right]=5.3 \times 10^{-42}\left(L_{\mathrm{H} \alpha}+0.031 L_{24}\right) .
$$

Here $L_{\mathrm{H} \alpha}$ is the line-integrated $\mathrm{H} \alpha$ luminosity, with units of $\mathrm{erg} \mathrm{s}^{-1}$ and no correction for internal extinction. $L_{24} \equiv$ $\nu L_{\nu}(24 \mu \mathrm{m})$ is the $24 \mu \mathrm{m}$ luminosity, also in units of $\mathrm{erg} \mathrm{s}^{-1}$. This luminosity comes from multiplying the specific luminosity, $L_{\nu}$, by the frequency at $\lambda=24 \mu \mathrm{m}$.

For a measured line-integrated intensity, $I_{\mathrm{H} \alpha}$, in $\operatorname{erg~s}^{-1} \mathrm{~cm}^{-2} \mathrm{sr}^{-1}$, across a beam with angular area $\Omega$ at distance $d$, the corresponding SFR surface density, $\Sigma_{\mathrm{SFR}}$, is

$$
\begin{gathered}
\Sigma_{\mathrm{SFR}}=\frac{\mathrm{SFR}}{\Omega d^{2}}, \\
=\frac{5.3 \times 10^{-42} I_{\mathrm{H} \alpha} \Omega 4 \pi d^{2}}{\Omega d^{2}} .
\end{gathered}
$$

Adopting the standard units for $\Sigma_{\mathrm{SFR}}$ of $M_{\odot} \mathrm{yr}^{-1} \mathrm{kpc}^{-2}$, the $d$ in the numerator is in $\mathrm{cm}$, whereas the $d$ in the denominator is in $\mathrm{kpc}$. We correct this unit inconsistency by multiplying the numerator by $9.52 \times 10^{42} \mathrm{~cm}^{2} \mathrm{kpc}^{-2}$. This yields $\Sigma_{\mathrm{SFR}}=$ $634 I_{\mathrm{H} \alpha}$ with $\Sigma_{\mathrm{SFR}}$ in $M_{\odot} \mathrm{yr}^{-1} \mathrm{kpc}^{-2}$ and $I_{\mathrm{H} \alpha}$ in $\mathrm{erg} \mathrm{s}^{-1} \mathrm{~cm}^{-2} \mathrm{sr}^{-1}$.

To place the $24 \mu \mathrm{m}$ term in surface brightness units, we combine the 634 prefactor from the previous calculation, the 0.031 relative weighting of luminosities from Equation (2), the conversion of $1 \mathrm{MJy} \mathrm{sr}^{-1}=10^{-17} \mathrm{erg} \mathrm{s}^{-1} \mathrm{~cm}^{-2} \mathrm{sr}^{-1} \mathrm{~Hz}^{-1}$, and $\nu(24 \mu \mathrm{m}) \approx 1.25 \times 10^{13} \mathrm{~Hz}$. Then

$$
\Sigma_{\mathrm{SFR}}=634 I_{\mathrm{H} \alpha}+0.0025 I_{24 \mu \mathrm{m}},
$$

again with $\Sigma_{\mathrm{SFR}}$ in $M_{\odot} \mathrm{yr}^{-1} \mathrm{kpc}^{-2}, I_{\mathrm{H} \alpha}$ in $\mathrm{erg} \mathrm{s}^{-1} \mathrm{~cm}^{-2} \mathrm{sr}^{-1}$, and $I_{24} \mu \mathrm{m}$ in $\mathrm{MJy} \mathrm{sr}^{-1}$.

We measure $24 \mu \mathrm{m}$ intensity from the SINGS maps (FWHM $\sim 6$ ". 4; Kennicutt et al. 2003). To estimate $I_{\mathrm{H} \alpha}$, we use the collection of literature $\mathrm{H} \alpha$ maps compiled and processed by Leroy et al. (2012). These draw heavily on LVL and SINGS (Kennicutt et al. 2003; Lee et al. 2009). This estimate assumes a Kroupa initial mass function (IMF). The correction to a Chabrier IMF is modest.

We convolve both the $\mathrm{H} \alpha$ and $24 \mu \mathrm{m}$ maps to the $8^{\prime \prime}$ resolution of the millimeter-wave data. To convolve the $24 \mu \mathrm{m}$, we use the kernels of Aniano et al. (2011). Because of the extended structure of the $24 \mu \mathrm{m}$ point-spread function (PSF), translating from the native PSF to an $8^{\prime \prime}$ Gaussian requires the use of an "aggressive" kernel. This may be another reason to prefer the $70 \mu \mathrm{m}$ oriented alternative described below.

In appendix, we show that in our sources and target regions the $24 \mu \mathrm{m}$ term dominates $\Sigma_{\mathrm{SFR}}$. $\mathrm{H} \alpha$ itself typically contributes $\sim 30 \%$ to the overall estimate.

\subsubsection{Indirect Total-infrared-based SFR Estimates}

As an alternative to the $\mathrm{H} \alpha+24 \mu \mathrm{m}$ approach, appendix works out an SFR estimate based on the TIR emission in our targets. We match the resolution of the Spitzer and Herschel maps at $24,70,160$, and $250 \mu \mathrm{m}$ at $30^{\prime \prime} .{ }^{18}$ Then, we use the prescriptions of Galametz et al. (2013) to calculate $\Sigma_{\text {TIR }}$, the TIR luminosity surface density. This corresponds to the expected integral under the whole IR spectral energy distribution from 8 to $1000 \mu \mathrm{m}$. If the light in the region under consideration is dominated by an embedded young stellar population, then this offers an alternative tracer of recent star formation.

The full suite of IR bands is only available at poor $\left(22^{\prime \prime}\right)$ resolution. To estimate the small-scale behavior of $\Sigma_{\mathrm{TIR}}$, we use the high-resolution $70 \mu \mathrm{m}$ maps from Herschel (Kennicutt et al. 2011). In appendix, we show that $70 \mu \mathrm{m}$ emission correlates better with $\Sigma_{\text {TIR }}$ than the $24 \mu \mathrm{m}$ does at low resolution. We calibrate a simple conversion from $70 \mu \mathrm{m}$ to TIR for each galaxy in our sample. This conversion is not perfect, but assuming that it holds subresolution allows us to estimate $\Sigma_{\text {TIR }}$ at the $8^{\prime \prime}$ common resolution of our millimeterwave data.

We present these "TIR"-based estimates in the table or radial profiles, adopting the conversion from $\Sigma_{\text {TIR }}$ to $\Sigma_{\text {SFR }}$ calculated by Murphy et al. (2011):

$$
\left(\frac{\Sigma_{\mathrm{SFR}, \mathrm{TIR}}}{M_{\odot} \mathrm{yr}^{-1} \mathrm{kpc}^{-2}}\right)=1.48 \times 10^{-10}\left(\frac{\Sigma_{\mathrm{TIR}}}{L_{\odot} \mathrm{kpc}^{-2}}\right) \text {. }
$$

Murphy et al. (2011) derive this conversion assuming that the entire Balmer continuum is absorbed and re-radiated by dust and assuming a continuous star formation history with no contamination due to an old background stellar population. Based on comparison with UV emission, all of our targets appear heavily extinguished across the region of interest (so that adding a GALEX-based term would not influence $\Sigma_{\mathrm{SFR}, \mathrm{TIR}}$ significantly). However, variations in the star formation history or heating by older stellar populations remain a concern (though these are among the most gas-rich, IR-bright, actively star-forming regions in the KINGFISH Herschel survey).

This TIR estimate follows a less conventional approach than the $\mathrm{H} \alpha+24 \mu \mathrm{m}$, but it represents our preferred SFR estimate for comparison to the literature. We recommend adopting these values from the table of radial profiles when carrying out such a

\footnotetext{
18 The common $30^{\prime \prime}$ resolution was chosen based on the ability to compare to IRAM $30 \mathrm{~m}$ maps for a related project. In principle, this comparison could be done at 22" (FWHM) resolution. The Herschel $250 \mu \mathrm{m}$ PSF can be "very safely" convolved to a Gaussian with this resolution (see Aniano et al. 2011).
} 
comparison. However, in the main text, we will plot results deriving SFR from $\mathrm{H} \alpha+24 \mu \mathrm{m}$.

\subsubsection{Literature Star Formation Rate Estimates}

We compare our results to those of Usero et al. (2015), Gao \& Solomon (2004), and García-Burillo et al. (2012). Each of these estimates the TIR luminosity and uses this as their SFR indicator. But each study calculates TIR slightly differently. Usero et al. (2015) calculate TIR from combining 24-160 $\mu \mathrm{m}$ data to get a $24 \mu \mathrm{m} / \mathrm{TIR}$ ratio at coarse resolution, and then they rescale the $24 \mu \mathrm{m}$ intensity at their working resolution by that factor (similar to our approach in appendix). Gao \& Solomon (2004) calculate TIR luminosity for their whole galaxies using the prescription in Sanders \& Mirabel (1996) and measurements from all four IRAS flux bands-12, 25, 60, and $100 \mu \mathrm{m}$. García-Burillo et al. (2012) calculate TIR intensity using a scaling of 60-100 $\mu \mathrm{m}$ from Graciá-Carpio et al. (2008). Based on appendix, we expect these different approaches to introduce scatter into any comparison at the $\sim 30 \%$ level. Homogenizing the literature SFR estimates used in dense gas studies clearly has large value but is beyond the scope of this study.

\subsection{Stellar Mass}

Usero et al. (2015) and Bigiel et al. (2016) highlighted strong correlations with stellar structure, a possible driver of gas pressure. With this in mind, we compare our observations to $3.6 \mu \mathrm{m}$ maps, which are dominated by light from old stars. We use maps obtained by Spitzer, mostly as part of SINGS (Kennicutt et al. 2003), and processed as part of the $S^{4} G$ survey (Sheth et al. 2010). Because Spitzer's $3.6 \mu \mathrm{m}$ band can include emission associated with recent star formation, primarily dust emission, we use the contaminant-corrected maps of Querejeta et al. (2015), which are based on an algorithm developed by Meidt et al. (2012). Globally, the correction of Querejeta et al. (2015) and Meidt et al. (2012) attributes $10 \%-30 \%$ of the $3.6 \mu \mathrm{m}$ emission to a component associated with star formation and removes it from the maps. The PSF of the IRAC $3.6 \mu \mathrm{m}$ maps has FWHM 1."9 before any convolution.

We calculate the stellar surface density from the contaminant-corrected $3.6 \mu \mathrm{m}$ data (Querejeta et al. 2015) assuming a mass-to-light ratio of $352 \mathrm{M}_{\odot} \mathrm{pc}^{-2}\left(\mathrm{MJy} \mathrm{sr}^{-1}\right.$ ) (Meidt et al. 2014). This roughly corresponds to $0.5 M_{\odot}$ per $L_{\odot}$. Note that Usero et al. (2015) and Bigiel et al. (2016) used median-filtered versions of the $3.6 \mu \mathrm{m}$ maps to correct for contamination, rather than the ICA-corrected versions from Querejeta et al. (2015). Usero et al. (2015) also used a different mass-to-light ratio. After accounting for the different mass-to-light ratio, the contaminant-corrected $3.6 \mu \mathrm{m}$ map that we use here agrees reasonably with the median-filtered Usero et al. (2015) and Bigiel et al. (2016) data.

\subsection{Atomic Gas Mass}

The distribution of atomic gas ( $\mathrm{HI}$ ) plays a role in calculating dynamical equilibrium pressure. We compare to the H I maps from THINGS (Walter et al. 2008) for NGC 3351, NGC 3627, and NGC 5194 and archival VLA maps for NGC 4254 and NGC 4321 (from Schruba et al. 2011; Leroy et al. 2013b). We begin with the $21 \mathrm{~cm}$ integrated intensity ("moment 0") maps and convert these to hydrogen column density assuming optically thin emission (see Walter et al. 2008).

The H I maps have coarser resolution than our other data. The naturally weighted THINGS maps for NGC 3351 and NGC 3627 have synthesized beams of 9 !! $9 \times 7$ !" 1 and 10 " $0 \times 8$ "! 9 . The archival maps of NGC 4254 and NGC 4321 have synthesized beams of 16 !" $9 \times 16$ !" 2 and 14 ! $7 \times 14$ !" 1 . In all cases, the $21 \mathrm{~cm}$ map covers the full spectral extent of the galaxy, and the pixels heavily oversample the synthesized beam. However, we note that the VLA maps do not include zero-spacing data and can be expected to filter out emission with angular extent $\gtrsim 30^{\prime}$ in an individual channel. Despite this, Walter et al. (2008) show that for the THINGS maps the agreement between the flux measured in the interferometer-only cube and single-dish measurements is quite good. In practice, we suggest to consider the zero level of these profiles to be perhaps biased slightly low, by $\lesssim 2 M_{\odot} \mathrm{pc}^{-2}$.

In our analysis, we will assume the $\mathrm{HI}$ to be smooth subresolution. This agrees with analysis showing the $\mathrm{H}$ I to be only weakly clumped (Leroy et al. 2013a) and with the relatively flat shape of the radial profiles (Schruba et al. 2011; see below). The main application of the H I profiles will be to calculate total gas surface density for use in pressure estimates, so the analysis is not terribly sensitive to this assumption.

\subsection{Dynamical Equilibrium}

Usero et al. (2015) and Bigiel et al. (2016), following Helfer \& Blitz (1997), suggested that ISM pressure may play a central role in determining the gas density distribution and the role of gas at any particular density in star formation. We estimate the approximate midplane gas pressure needed to maintain a vertical dynamical equilibrium following Elmegreen (1989), Blitz \& Rosolowsky (2006), Wong \& Blitz (2002), Leroy et al. (2008), and Ostriker et al. (2010). Specifically, we take

$$
P_{\mathrm{DE}} \approx \frac{\pi G \Sigma_{\mathrm{gas}}^{2}}{2}+\Sigma_{\mathrm{gas}}\left(2 G \rho_{\star}\right)^{1 / 2} \sigma_{\mathrm{gas}, z} .
$$

Here $\Sigma_{\text {gas }}$ is the total atomic plus molecular gas surface density, $\rho_{\star}$ is the volumetric mass density of stars and dark matter at the midplane, and $\sigma_{\mathrm{gas}, z}$ is the vertical velocity dispersion of the gas.

$P_{\mathrm{DE}}$ expresses the pressure needed to balance the vertical gravity on the gas in the galaxy disk. The first term in Equation (7) reflects the gas self-gravity, and the second term reflects the weight of the gas in the potential well of the stars. For our target regions, the stellar potential will exceed the gas self-gravity, and we expect the second term to be dominant.

Only the stars and dark matter within the gas layer are relevant to the potential. We expect the density of dark matter within the gas layer to be small compared to that of the stars, and so we neglect that contribution to $\rho_{\star}$. Thus, $\rho_{\star}$ rather than $\Sigma_{\star}$ enters Equation (7). Estimating the 3D structure of the disk represents the main challenge to gauging $P_{\mathrm{DE}}$ from observations. We need estimates of the local stellar scale height to translate our inferred $\Sigma_{\star}$ into $\rho_{\star}$ via

$$
\rho_{\star} \approx \frac{\Sigma_{\star}}{2 h_{\star}}
$$

following van der Kruit (1988).

A full treatment of the height of stellar disks as a function of morphology, mass, and galactocentric radius is beyond the scope of this paper. Observations still do not provide a perfect 
prescription, and more recent observations show that the topic may be more complex than suggested by earlier work (e.g., Comerón et al. 2012, 2014). Lacking a better prescription, we follow Leroy et al. (2008) and adopt the typical flattening ratio found by Kregel et al. (2002), $l_{*} / h_{*}=7.3 \pm 2.2$, to relate the stellar scale height and the observed scale length for the disk component.

We adopt $\sigma_{z \text {,gas }} \approx 15 \mathrm{~km} \mathrm{~s}^{-1}$. This is toward the high end of the observed gas velocity dispersions in galaxy disks, appropriate for large scales and the gas-rich, high surface density inner regions of galaxy disks (e.g., Tamburro et al. 2009; Caldú-Primo et al. 2013; Mogotsi et al. 2016).

For these assumptions, we calculate $P_{\mathrm{DE}}$ in each ring and report this in the online table described in Appendix C.

Finally, we provide a word on the interpretation of $P_{\mathrm{DE}} \cdot P_{\mathrm{DE}}$ is the time-averaged pressure needed to support the gas disk against its weight due to both self-gravity and the gravity of the stellar potential well. We include all gas in this calculation and expect Equation (7) to hold averaged over time and space (several gas scale heights), with all gas participating in this equilibrium. Thus, we expect $P_{\mathrm{DE}}$ to express the average state of a region of the ISM. Equation (7) does not distinguish between a gravitationally bound component and a diffuse component of the gas. This is consistent with recent simulations (e.g., Kim \& Ostriker 2017) and observations that indicate that self-gravitating structures are quite transient (Kawamura et al. 2009; Schruba et al. 2010). Such transient structures may be thought of as fully participating in the ISM's large-scale dynamics when averaged over several scale heights and vertical crossing times. Similarly, for a turbulencedominated system we do not think of $P_{\mathrm{DE}}$ as an external pressure that acts on cloud "surfaces"; rather, it is the mean pressure averaging over both high- and low-density regions.

\subsection{Convolution to a Common Resolution}

We convolved all data to a working resolution of $8^{\prime \prime}$, using the kernels provided by Aniano et al. (2011) when needed. After convolution, we aligned all of the broadband multiwavelength data to the common astrometric grid shared by the $\mathrm{HCN}, \mathrm{HCO}^{+}, \mathrm{CS},{ }^{13} \mathrm{CO}$, and $\mathrm{C}^{18} \mathrm{O}$ data. As mentioned above, the $\mathrm{H}$ I data could not be beam-matched to an $8^{\prime \prime}$ beam, and we use these at their native resolution, assuming a smooth distribution within the beam.

We use a combination of the convolved Spitzer $24 \mu \mathrm{m}$ data and $\mathrm{H} \alpha$ data as our primary SFR tracer. Despite the modest $\sim 6^{\prime \prime}$ core of the $24 \mu \mathrm{m}$ PSF, the Spitzer PSF has significant extended structure, and an "aggressive" kernel is required to convolve a $24 \mu \mathrm{m}$ image to have an $8^{\prime \prime}$ Gaussian PSF (Aniano et al. 2011). As a result, mild artifacts are visible surrounding the nucleus of NGC 3351 in the convolved $24 \mu \mathrm{m}$ image. The alternative $70 \mu \mathrm{m}$ based tracer has a cleaner PSF. Here again, comparing the two approaches offers a useful way to assess systematic effects.

Note that we use this $8^{\prime \prime}$ working resolution for NGC 3351 , NGC 3627, NGC 4254, and NGC 4321. We use a lower 30" working resolution for NGC 5194, set by the resolution of the IRAM $30 \mathrm{~m}$ data. Otherwise, the procedure is the same.

\subsection{Construction of Radial Profiles}

The most striking contrast in our data is between the bright central regions and the surrounding disk, which includes fainter emission from gas in the spiral arms and bars. Even ALMA struggles to detect emission from the extended disk at high significance. To improve our $\mathrm{S} / \mathrm{N}$, we conduct much of our analysis using radial profiles of line emission and supporting data. Although azimuthal (e.g., arm-interarm) contrast will also be interesting for future studies, our present data lack the sensitivity to recover faint interarm emission.

We set the width of each radial bin to be half the FWHM of the working beam (15" for NGC 5194 and $4^{\prime \prime}$ for all other galaxies). This should critically sample the radial structure of the galaxy. It also yields profiles in which adjacent rings are correlated, so that we have twice as many data in each plot than independent radial measurements.

In each ring, we calculate two values: (1) the mean intensity of all pixels within the 2D version of the $\mathrm{CO}$ mask, ignoring all other values, and (2) the mean intensity of all pixels in the ring. For profile 2, we treat pixels outside the map as having intensity 0 for the molecular lines, so that the ring-averaged value represents the values in the mask diluted by the empty space outside the mask. For the ancillary data $(3.6 \mu \mathrm{m}, \mathrm{H} \alpha$, $24 \mu \mathrm{m}$, etc.), we calculate the mean value only within the $2 \mathrm{D}$ CO-based mask for profile 1 and the mean value over the whole ring for profile 2 . We report both values in the table described in Appendix C. Note that for these other data, we do not set any pixels to 0 when taking the mean over the whole ring, but instead use all of the data.

We calculate the uncertainty for these mean intensities by propagating the error from individual intensity measurements. In this case the uncertainty is

$$
\Sigma_{i i}=\frac{\sqrt{\sigma_{1}^{2}+\sigma_{2}^{2}+\ldots+\sigma_{N}^{2}}}{N} \times \sqrt{O} .
$$

Here $\Sigma_{i i}$ is the uncertainty in the integrated intensity averaged over the ring, and $N$ is the number of pixels in the ring. $O$ is a factor (the "oversampling factor") designed to account for the nonindependence of the pixels. If all data are independent with the same uncertainty, $\sigma_{I I}=\sigma_{1} / \sqrt{N}$ as expected for the uncertainty in the mean of $N$ data. Generally $O$ should be the number of pixels per beam. Here, given that our rings are thinner than a beam, we take $O$ equal to the ring thickness in pixels times the FWHM beam size in pixels. We never allow $N / O<1$, so that $\sigma_{I I}$ is at most the uncertainty in an individual point. When calculating this mean intensity for the line data, pixels outside of the mask are ignored even for profile 2 . That is, our uncertainties do not reflect uncertainty in mask construction.

The intensity profiles of each line, along with profiles of the supporting multiwavelength data, are a main observational result of the paper. These are available in full as online material. All surface brightness and surface densities quoted in the paper have been corrected for the effects of inclination.

\section{Results}

Figures 1-5 show $\mathrm{CO}$ and $\mathrm{HCN}$ emission in context for our four ALMA targets and NGC 5194 (M51). The top left panel shows the distribution of dense gas traced 

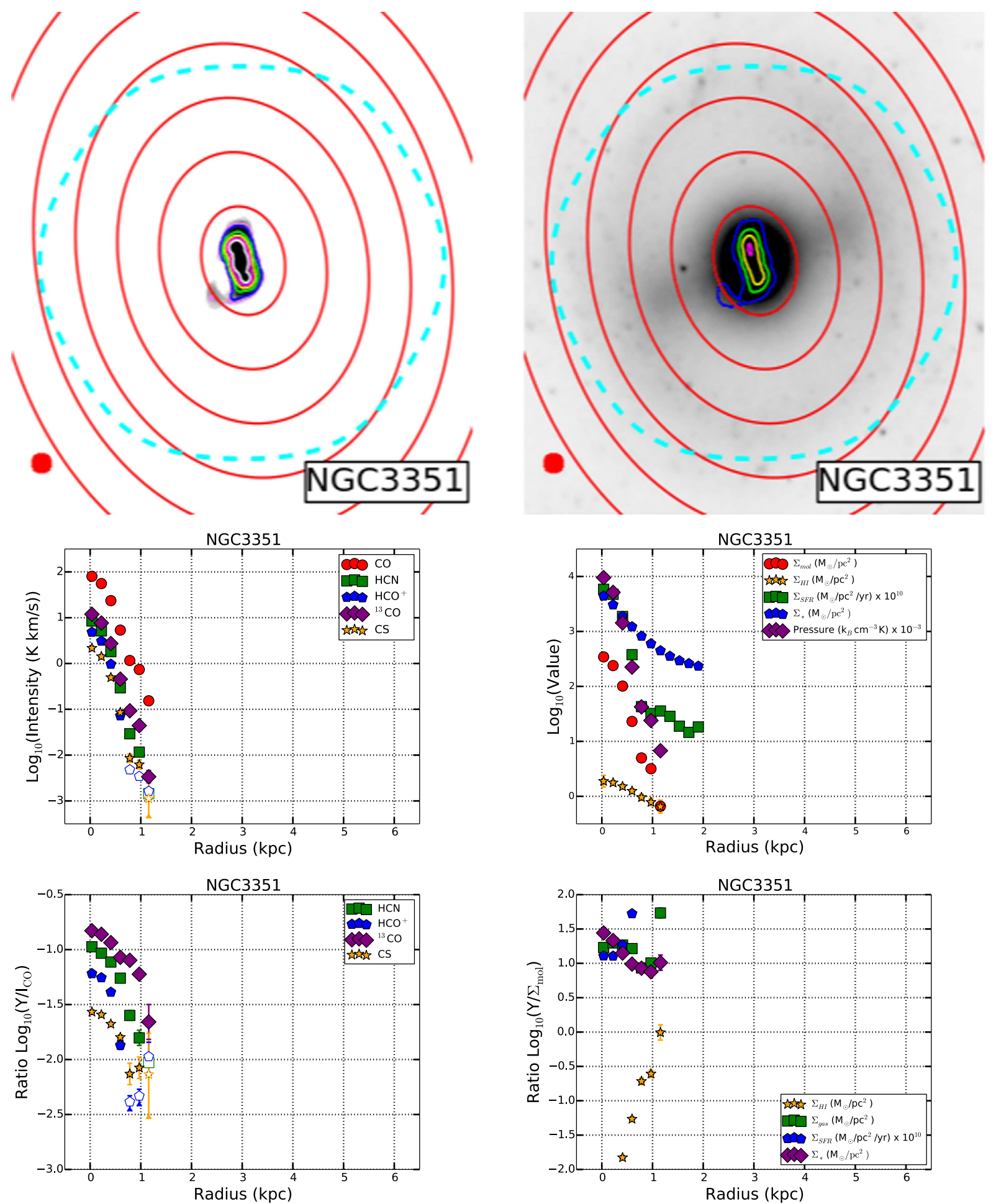

Figure 1. Our ALMA observations in context for NGC 3351. Top left: HCN contours (color) over CO integrated intensity (gray scale). HCN contours show 0.5 (violet), 1 (blue), 3.5 (green), 6.5 (yellow), 9.5 (magenta), and 12.5 (white) $\mathrm{K} \mathrm{km} \mathrm{s}^{-1}$. Red rings show fixed galactocentric radius in the plane of the galaxy, spaced by $1 \mathrm{kpc}$. The filled red circle in the lower left shows the common $\theta=8^{\prime \prime}(\mathrm{FWHM})$ beam used in our analysis. The dashed cyan contour shows the ALMA field of view for HCN emission. Top right: $3.6 \mu \mathrm{m}$ map, tracing stellar structure, with CO integrated intensity contours at $10,60,110,160$, and $210 \mathrm{~K} \mathrm{~km} \mathrm{~s}{ }^{-1}$. Middle left: azimuthally averaged integrated intensity profiles for $\mathrm{CO}, \mathrm{HCN}, \mathrm{CS}, \mathrm{HCO}^{+}$, and ${ }^{13} \mathrm{CO}$ (see Section 2.10), restricting the average to regions with statistically significant $\mathrm{CO}$ emission. Filled points have $\mathrm{S} / \mathrm{N} \geqslant 2$. Error bars show uncertainty in the mean. Open points indicate upper limits. Middle right: intensity profiles for tracers of galaxy and ISM structure: CO (tracing molecular gas), $\mathrm{H} \alpha$, and $24 \mu \mathrm{m}$ emission (tracing star formation), and contaminant-corrected $3.6 \mu \mathrm{m}$ emission (tracing stellar mass). Bottom left: ratio of other lines to CO intensity as a function of radius. Bottom right: ratios among tracers of galaxy and ISM structure, along with estimated dynamical equilibrium pressure. The radial profiles are available online as described in Appendix C.

by $\mathrm{HCN}$ (colored contours) over the distribution of total molecular gas, traced by $\mathrm{CO}$ (gray scale). The top right panel shows the total molecular gas distribution $(\mathrm{CO}$, color contours) and the stellar structure (traced by $3.6 \mu \mathrm{m}$ light in gray scale). In both panels, red ellipses indicate fixed galactocentric radius, allowing one to visually map to 

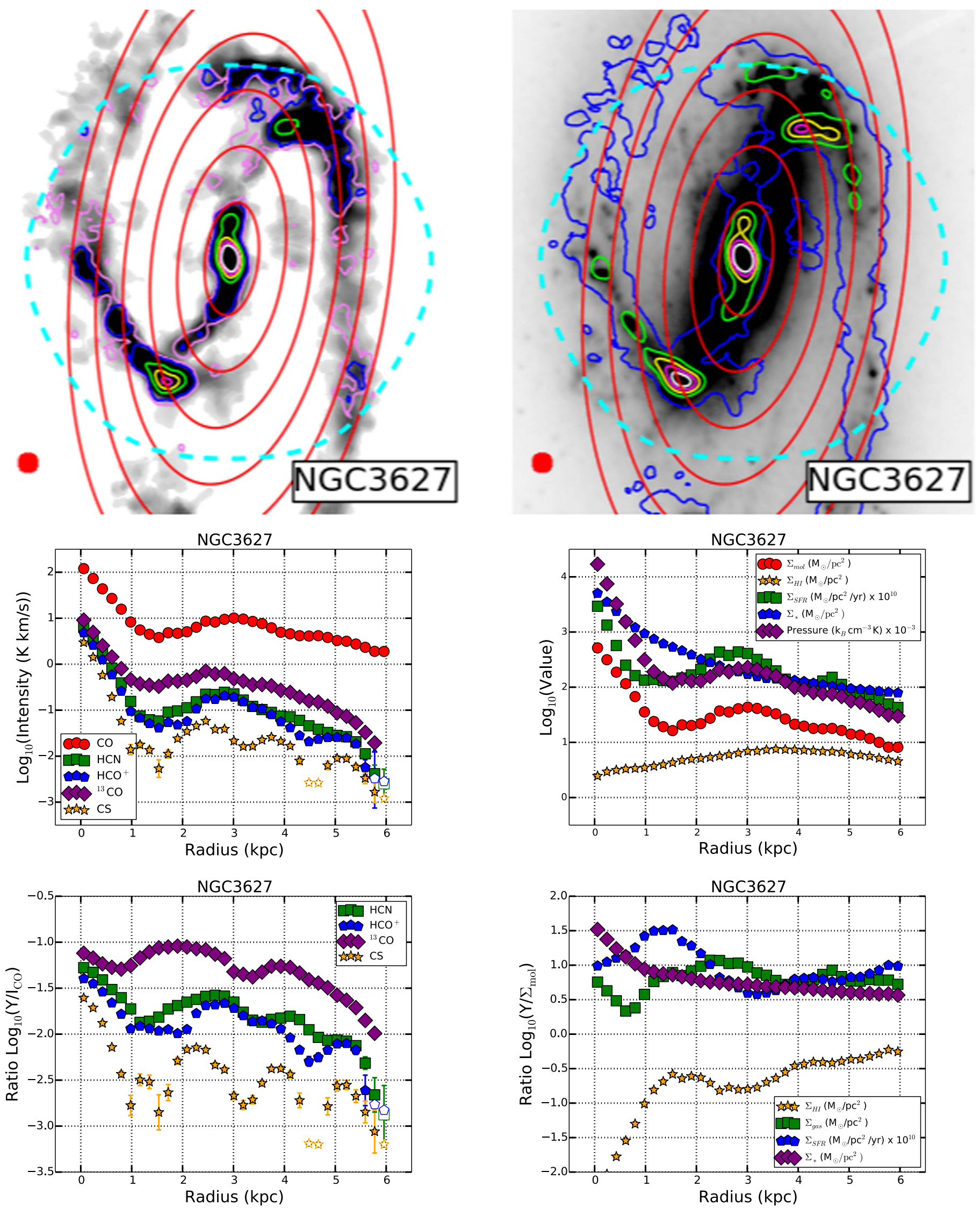

Figure 2. Same as Figure 1, but for NGC 3627. 

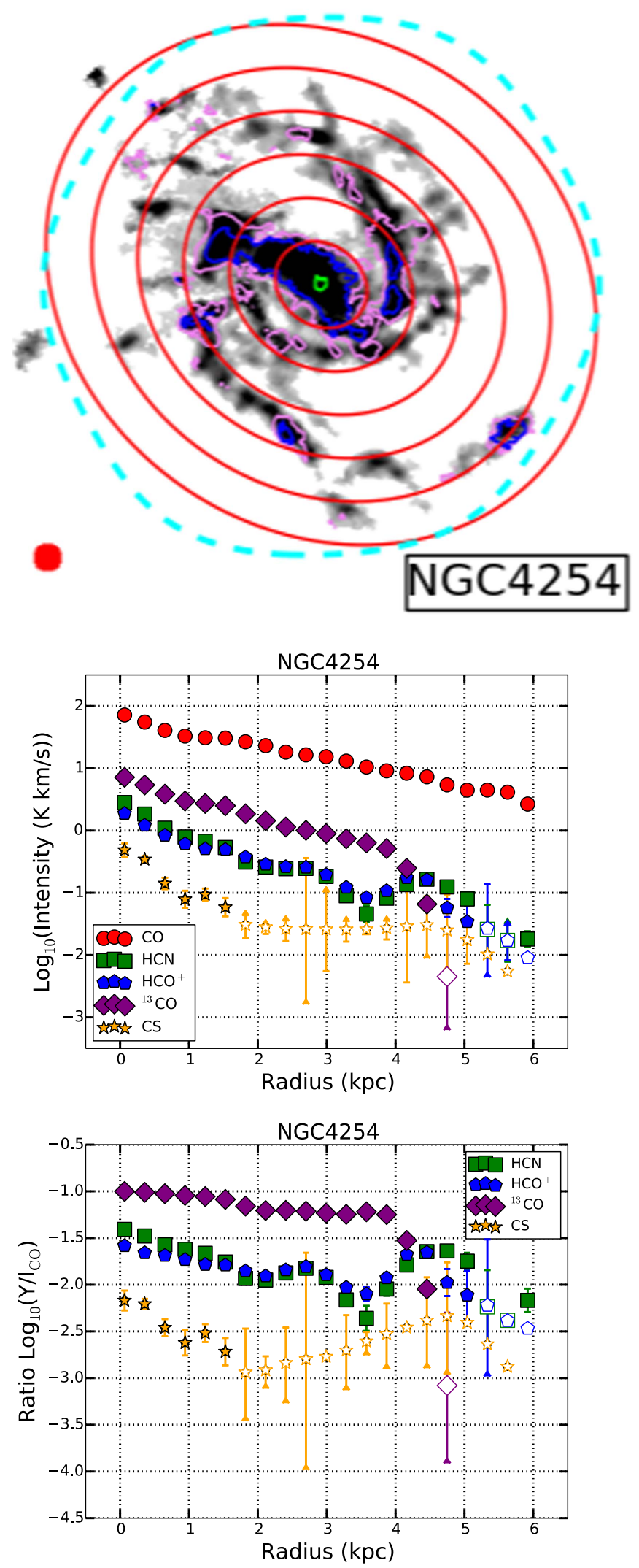
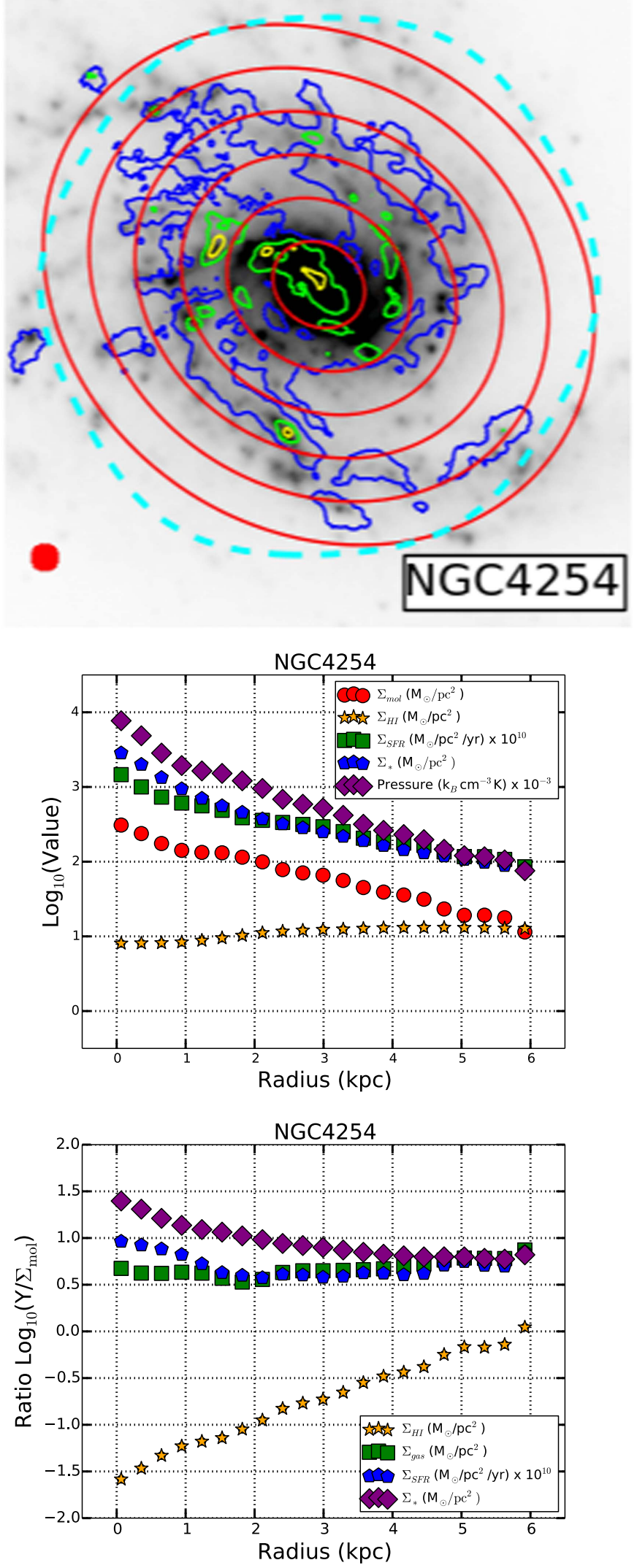

Figure 3. Same as Figure 1, but for NGC 4254. 

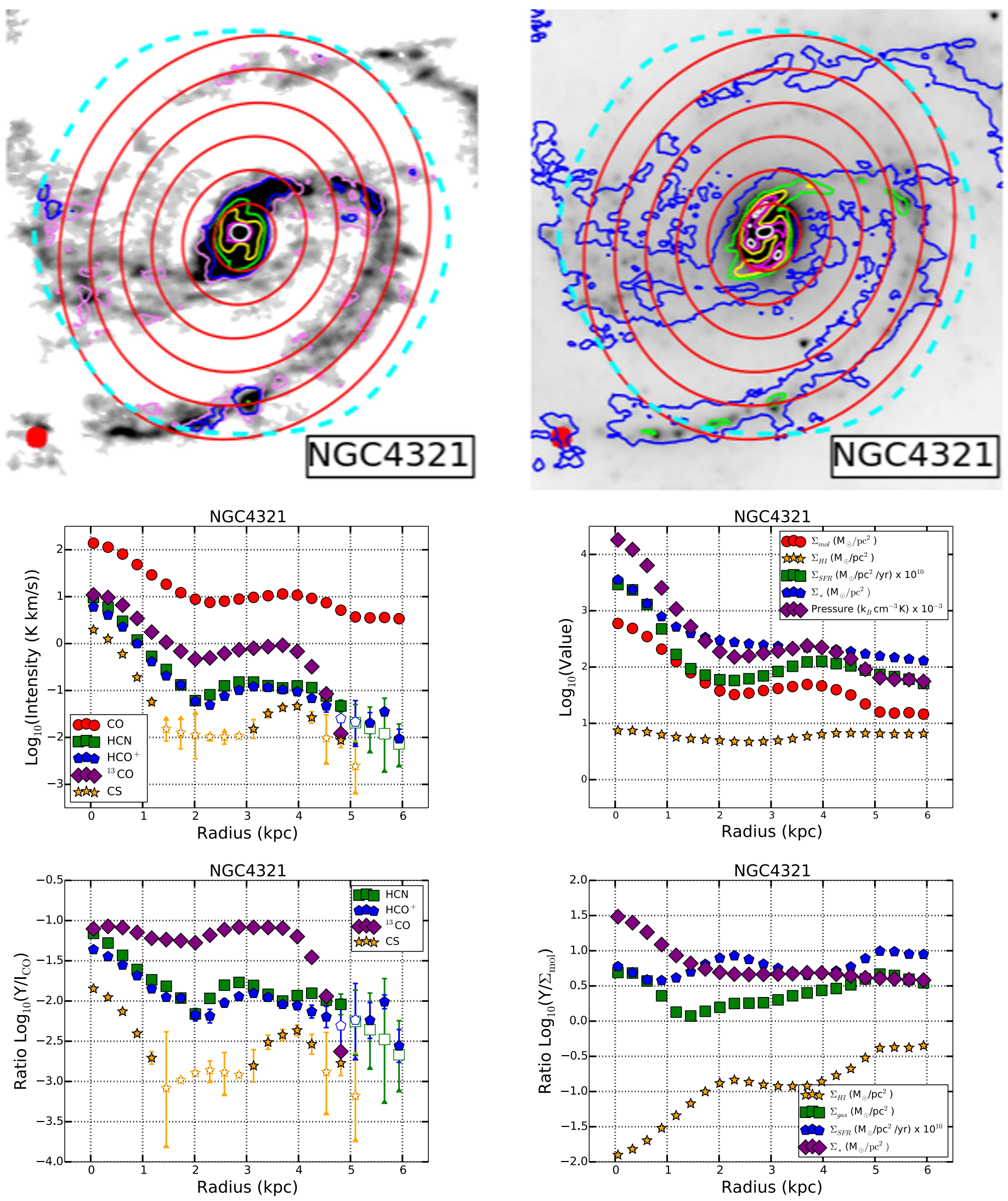

Figure 4. Same as Figure 1, but for NGC 4321.

the radial profiles below. Our maps extend to $r_{\text {gal }} \sim 3 \mathrm{kpc}$ in NGC 3351 and NGC 3627 and to $\sim 5 \mathrm{kpc}$ in NGC 4254, NGC 4321, and NGC 5194.
We see bright $\mathrm{CO}$ emission in the centers of all five galaxies and fainter $\mathrm{CO}$ emission out to many kiloparsecs along the bars and spiral arms of all targets except NGC 3351. NGC 3351 

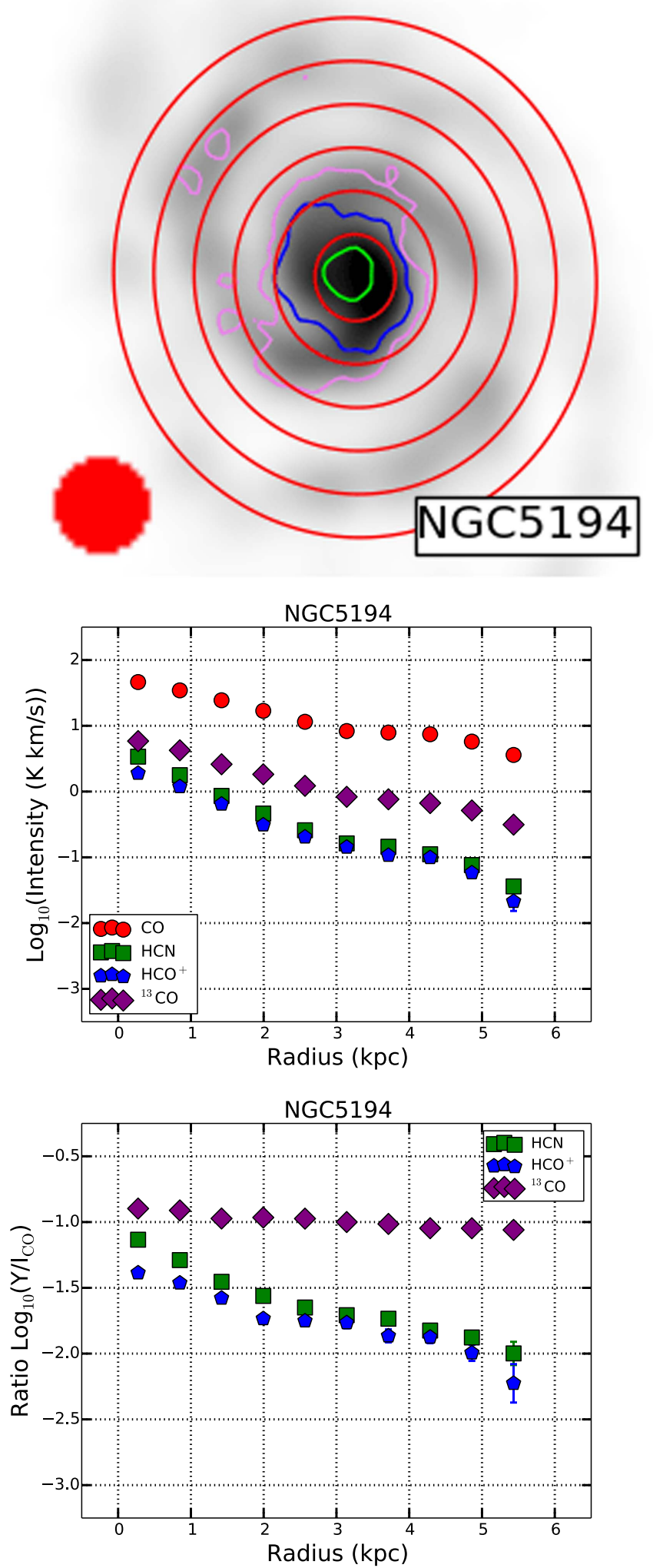
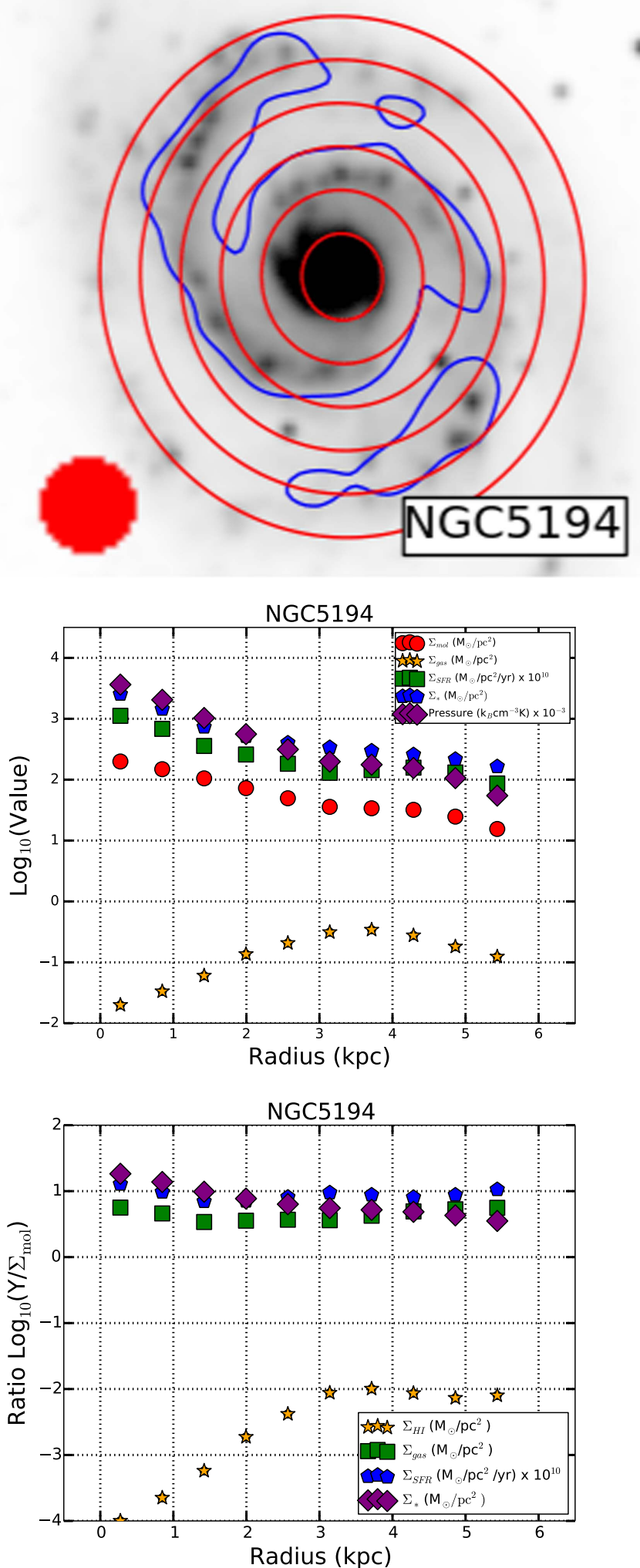

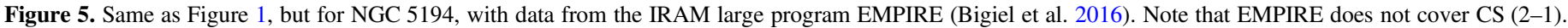
Please note that the $y$-axis range for the two right panels is different for NGC 5194 than for the other four galaxies.

shows a bright central disk of molecular gas, but little molecular gas across the stellar bulge. The galaxy has a ring of star formation and molecular material at $\sim 4 \mathrm{kpc}$ (e.g., Leroy et al. 2009), but this lies near the edge of our mosaic. This outer ring remains undetected in dense gas tracers in our observations and is faint compared to the sensitivity of the BIMA SONG CO (1-0) map shown here (Helfer et al. 2003). Thus, NGC 3351 appears as only a compact $(\lesssim 1 \mathrm{kpc})$ gas disk in our analysis. 
$\mathrm{HCN}$ emission follows the $\mathrm{CO}$, with bright $\mathrm{HCN}$ intensity $\left(I_{\mathrm{HCN}}\right)$ detected at high significance in the central regions of each galaxy. In the four targets with widespread $\mathrm{CO}$ emission, we also detect pervasive $\mathrm{HCN}$ emission at the $\sim 2 \sigma$ level throughout the bar and spiral arms. In the analysis below, we use the $\mathrm{CO}$ emission as a prior to make a clean measurement of $I_{\mathrm{HCN}}$. Before doing this, we also inspected the cubes for evidence of $\mathrm{HCN}$ and other molecules outside the region of bright $\mathrm{CO}$ emission. We do not see evidence for such emission at our sensitivity level.

These panels illustrate the close connection between molecular gas and stellar structure, which has been shown many times and we see again below (e.g., Young \& Scoville 1991; Regan et al. 2001; Leroy et al. 2009). CO emission is concentrated in the inner regions of the galaxy, where the stellar surface density is high, and follows the arms and bars in the three galaxies that show extended molecular gas.

\subsection{Radial Profiles}

Even with ALMA, emission from dense gas tracers remains hard to detect. To overcome this, we carry out most of our analysis using azimuthally averaged radial profiles (see Section 2.10). Note that the masked regions are zeros in this averaging. When we report ratios, these are the ratios among the azimuthal profiles taken after radial profile construction.

The middle panels in Figures 1-5 show these profiles for our target lines (left) and key measures of the galaxy and ISM structure (right). Even with azimuthal averaging, we still do not detect the fainter lines in some rings. In these cases we show the $2 \sigma$ value as an open symbol to indicate an upper limit.

The absolute intensities trace the distribution of material. To capture the changing physical state of the gas, the bottom rows of Figures 1-5 plot ratios among radial profiles. For the lines, we show the intensity of each relative to $\mathrm{CO}$. For the galaxy structure, we show the fractional content of gas (as opposed to stars), the rate of star formation per unit gas, and the balance between atomic and molecular gas. Note that we multiply $\Sigma_{\mathrm{SFR}}$ by a factor of $10^{10}$ to show it on the same scale as the other quantities.

\subsubsection{Galaxy and ISM Structure}

Three of our five galaxies have strong bars (Muñoz-Mateos et al. 2013) and also show strong nuclear concentrations of gas. NGC 4254, which lacks a strong bar, does not show such a feature. NGC 5194, which also lacks a strong bar, shows a weaker version of the feature. A natural explanation for the difference between the profiles of galaxies with strong bars and those without is that bar-induced streaming motions transport gas inward in the barred galaxies (e.g., Downes et al. 1996; Regan et al. 1999; Sheth et al. 2005). This leads to a higher concentration of gas in the inner region, which in turn leads to a higher rate of star formation in the inner $\sim 1 \mathrm{kpc}$ of these targets. The same mechanism has been used to explain the nuclear region in the Milky Way.

The radial profiles in Figures 1-5 show such nuclear enhancements. Within 1-2 kpc, NGC 3351, NGC 3627, NGC 4321, and NGC 5194 all exhibit high $\Sigma_{\text {gas }}, \Sigma_{\text {mol }}$, and $\Sigma_{\text {SFR }}$ relative to the surrounding disk. These central gas concentrations are a few hundred parsecs to a kiloparsec in extent and result in sharply declining profiles out to $r_{\text {gal }} \sim 2 \mathrm{kpc}$. Then they level out, declining more weakly or remaining flat to the edge of our field of view. Recall that these figures show profiles averaging only where $\mathrm{CO}$ is detected, so empty space, e.g., between arms, is not reflected in the profiles. The stellar surface density, $\Sigma_{*}$, exhibits a similar two-zone behavior, but with a smoother transition between regimes.

The impact of bars is also visible in the images. The top right panels show CO contours over the stellar distribution. Increased gas surface density along an inner bar is visible in NGC 3627 and NGC 4321. In NGC 3351, a strong bar visible in the near-IR links the ring of star formation and molecular gas to the central disk, but our observations detect little gas between the ring and the bar. NGC 5194 shows a comparatively weaker bar than our other targets. Furthermore, the lower resolution for this galaxy makes it hard to compare structural elements between it and the other four galaxies. However, the inner parts of the spiral arms may play some of the same role as a bar, funneling material toward the inner galaxy (e.g., Meidt et al. 2013; Querejeta et al. 2016).

Distinct from our other targets, NGC 4254 shows radial profiles that extend continuously from the central part of the galaxy out into the disk. The central brightness of $\mathrm{CO}$ emission is still high in this galaxy, comparable to our other targets. However, the profiles exhibit no strong evidence for a physically discrete nuclear concentration of gas. This galaxy also lacks a strong bar; it is the only galaxy in our sample not identified to have a bar in RC3 or by Muñoz-Mateos et al. (2013). This makes the contrast between NGC 4254 and the other three galaxies a useful point of comparison.

Figures 1-5 show that emission from the dense gas tracers is also centrally concentrated in our barred targets. Indeed, we will see below that $\mathrm{HCN}$ emission appears even more concentrated than CO. Previous work suggests that the gas in these nuclear concentrations also tends to be more excited (see, e.g., Braine \& Combes 1992; Leroy et al. 2009, 2013b) and may have a lower $\mathrm{CO}-$ to- $\mathrm{H}_{2}$ conversion factor (e.g., Sandstrom et al. 2013) than gas at larger galactocentric radii.

This concentration of gas and star formation resembles a similar feature seen in another strongly barred galaxy: the Milky Way. In the Milky Way, the central molecular zone that covers the inner few hundred parsecs hosts a large concentration of gas, an overabundance of dense gas, and substantial star formation (though not in a normalized sense; see Longmore et al. 2013). NGC 3351 may be a particularly striking counterpart, because, like the Milky Way, it appears deficient in gas along the bar through the bulge, but rich in gas in the inner region, with a ring of molecular material near the end of the bar (see, e.g., review by Heyer \& Dame 2015). However, NGC 3351 lacks the bar end starbursts believed to exist in the Milky Way, which do appear, e.g., in NGC 3627 (see also Beuther et al. 2016).

Radial profiles on the right-hand side of Figures 1-5 show that molecular (and not atomic) gas makes up the overwhelming fraction of the ISM over the fields that we study. We also see that, everywhere we have measurements, stars make up most of the baryonic matter in the disk. The profiles also show variations in the IR-to-CO ratio among and between our targets, tracing the rate at which gas forms stars.

\subsubsection{Line Ratio Profiles}

The middle left panels show the radial profiles for $\mathrm{CO},{ }^{13} \mathrm{CO}$, $\mathrm{HCO}^{+}, \mathrm{CS}$, and $\mathrm{HCN}$. Table 4 reports mean line ratios for our 
Table 4

Mean Line Ratios

\begin{tabular}{lcc}
\hline \hline Line & \multicolumn{2}{c}{ Ratio with $\mathrm{CO}^{\mathrm{a}}$} \\
\cline { 2 - 3 } & $r_{\text {gal }} \leqslant 1 \mathrm{kpc}$ & $r_{\text {gal }} \geqslant 1 \mathrm{kpc}$ \\
\hline${ }^{13} \mathrm{CO}$ & 0.088 & 0.039 \\
$\mathrm{HCO}^{+}$ & 0.026 & 0.008 \\
$\mathrm{CS}^{\mathrm{b}}$ & 0.011 & 0.001 \\
$\mathrm{HCN}$ & 0.046 & 0.01 \\
\hline
\end{tabular}

Note.

${ }^{a}$ Mean ratio over our four ALMA targets and NGC 5194.

${ }^{\mathrm{b}}$ For CS we lack short-spacing data and report values only for the four ALMA targets.

targets for $r_{\text {gal }} \leqslant 1 \mathrm{kpc}$ and $r_{\text {gal }} \geqslant 1 \mathrm{kpc}$. After $\mathrm{CO}$, the order of intensity from brightest to faintest is ${ }^{13} \mathrm{CO}, \mathrm{HCN}, \mathrm{HCO}^{+}$, and $\mathrm{CS}$. This rank order does not change substantially across the sample, though the relative brightnesses of the lines do vary. The two most common dense gas tracers, $\mathrm{HCN}$ and $\mathrm{HCO}^{+}$, have comparable intensities throughout our sample, though their absolute brightness and brightness relative to $\mathrm{CO}$ do vary from galaxy to galaxy and with radius (see also Usero et al. 2015; Bigiel et al. 2016). It is noteworthy that HCN and $\mathrm{HCO}^{+}$trace each other so closely. We discuss this more in Section 3.3.

This suite of lines traces gas with a range of densities (see Table 1). We utilize the ratios among lines with different $n_{\text {eff }}$ to infer changes in the sub-beam density distribution. To first order, the ratios of $\mathrm{HCN}, \mathrm{HCO}^{+}$, and $\mathrm{CS}$ to $\mathrm{CO}$ trace the fraction of dense molecular gas (for more discussion, see Section 3.3). The ratio of ${ }^{13} \mathrm{CO}$ to $\mathrm{CO}$ traces a combination of optical depth, isotopic ratio, and perhaps density of the bulk CO-emitting gas.

The bottom left panels of Figures 1-5 show the ratios between the mean intensity of each molecular line and that of CO. Measured line ratios vary within and among our sample, indicating changing physical conditions in the gas. In the three dense gas ratios, we see similar structure to that of the mean intensity radial profiles. We find inner regions with high ratios of dense gas tracer to $\mathrm{CO}$ emission, and this ratio declines as one moves out into the disk.

This decrease in apparent dense gas fraction occurs in all of our targets. In the two galaxies with bars and well-detected disk emission (NGC 3627 and NGC 4321), HCN, $\mathrm{HCO}^{+}$, and CS emissions all decrease relative to $\mathrm{CO}$ as one moves from the inner region out to the disk. Outside $r_{\text {gal }} \sim 1-2 \mathrm{kpc}$, these ratios flatten, remaining approximately constant at values $\sim 2-4$ times lower than observed in the inner region of the same galaxy.

Despite the lack of a distinct inner region in the radial profiles of NGC $4254, I_{\mathrm{HCN}} / I_{\mathrm{CO}}$ and $I_{\mathrm{HCO}+} / I_{\mathrm{CO}}$ also decline with increasing $r_{\text {gal }}$. This is consistent with results for NGC 5194 by Bigiel et al. (2016) and Chen et al. (2015). Like NGC 4254, NGC 5194 is not strongly barred, but it still shows declining density between the inner and outer parts of the galaxy. An anticorrelation between dense gas fraction and galactocentric radius thus appears to be a general feature, not exclusively a product of bar-driven flows. However, when a strong bar is present, its imprint does appear to be visible in the $I_{\mathrm{HCN}} / I_{\mathrm{CO}}$ profile.

\subsection{Dense Gas and Star Formation}

\subsubsection{Dense Gas-Star Formation Correlation}

We obtained these observations to test the idea that the dense gas fraction determines the star formation efficiency of molecular gas, $\mathrm{SFE}_{\mathrm{mol}} \equiv \mathrm{SFR} / M_{\mathrm{mol}}$. In a simple threshold model, the mass of dense gas determines the SFR. Such a relationship would explain the striking correlation between TIR luminosity (commonly used to trace star formation in the HCN literature) and HCN luminosity (tracing dense gas). This correlation extends from Galactic cores all the way to starburst galaxies (e.g., Gao \& Solomon 2004; Brouillet et al. 2005; Wu et al. 2010; Rosolowsky et al. 2011; García-Burillo et al. 2012; Kepley et al. 2014; Bigiel et al. 2015, 2016; Usero et al. 2015; see Figure 6).

To place our rings on this correlation, we translate our SFRs calculated from $\mathrm{H} \alpha+24 \mu \mathrm{m}$ to the corresponding TIR luminosity expected from an embedded population with this SFR (using Equation (6)). Though indirect, this approach is at least internally consistent with the fiducial SFR estimates in the main text. We use this conversion only for Figure 6. An alternative approach, using our best estimates of the local TIR luminosity based on $70 \mu \mathrm{m}$ emission (Section 2.5.2), yields similar results and can be carried out with the data provided in the table or profiles.

Figure 6 shows that our rings do populate the HCN-IR luminosity correlation seen at smaller and larger scales (Gao \& Solomon 2004; Lada et al. 2012). Our new data occupy the intermediate regime between whole galaxies and Milky Way cores or individual GMCs. In this, they resemble results for the disk pointings of Usero et al. (2015), the 30" NGC 5194 data of Bigiel et al. (2016) and Chen et al. (2015), and recent studies of M82 and the Antennae galaxies (Kepley et al. 2014; Bigiel et al. 2015). Our spatial resolution is finer than that of Bigiel et al. (2016), but our use of rings raises the luminosity in any individual data point.

Taking all of the literature data together, Figure 6 shows that that SFR correlates with HCN luminosity, with about a factor of two scatter across almost 10 orders of magnitude. Even if the luminosity of a specific ring in a galaxy lacks physical meaning, the narrow spread in the TIR-to-HCN ratio is significant. The right panel shows that over a large range, most data in the literature exhibit TIR-to-HCN ratios within $\sim \pm 0.3$ dex of one another. Although systematic differences are already evident both within and among studies, there is clearly a strong relationship between $\mathrm{HCN}$ luminosity and recent star formation.

\subsubsection{Does the Dense Gas Fraction Predict the Star Formation Efficiency of Molecular Gas?}

One popular approach to thinking about star formation, following, e.g., Gao \& Solomon (2004) and Lada et al. (2012), is to imagine a gas density threshold, above which the SFR scales with the mass of dense gas. In such a model, the star formation per unit mass of total molecular gas can and does vary, but star formation will correlate tightly with the mass of dense gas traced, e.g., by HCN. In such a model, any variation in $\mathrm{SFR}_{\mathrm{mol}} \equiv \Sigma_{\mathrm{SFR}} / \Sigma_{\mathrm{mol}}$ should be due to variations in $f_{\text {dense }}$ (traced here with $I_{\mathrm{HCN}} / I_{\mathrm{CO}}$ ).

We explore this prediction in Figure 7 . We show $\Sigma_{\text {SFR }} / \Sigma_{\text {mol }}$, or the overall efficiency of star formation, as a function of 

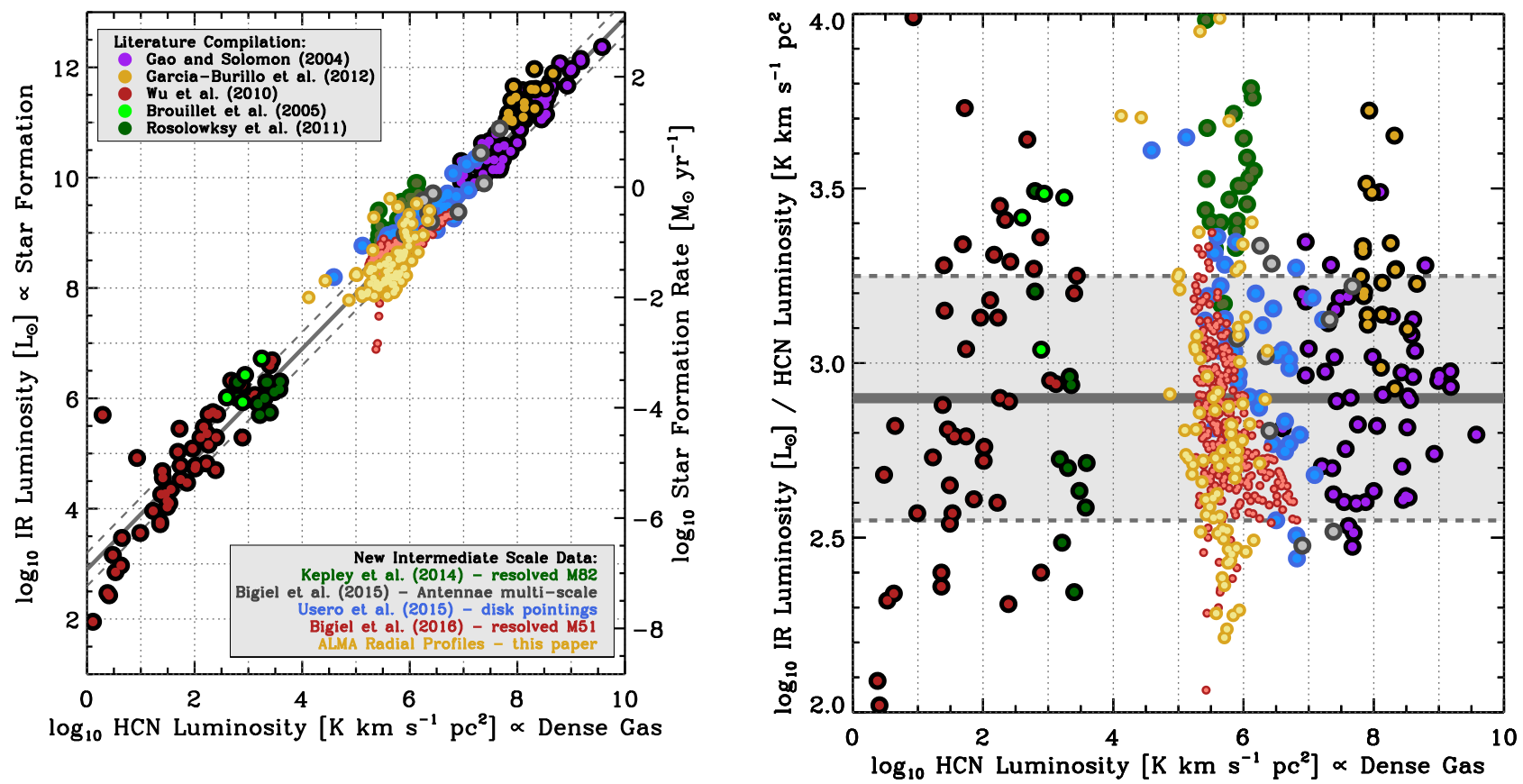

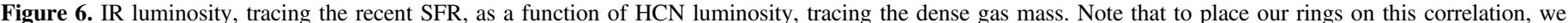

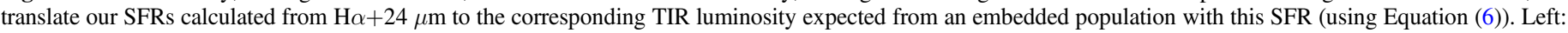

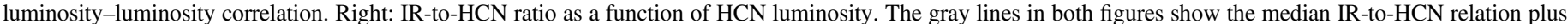

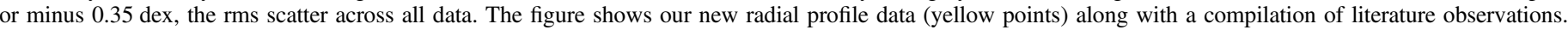

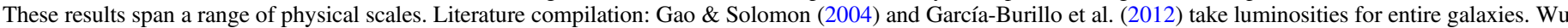

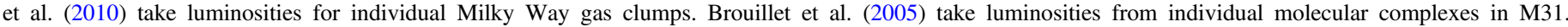

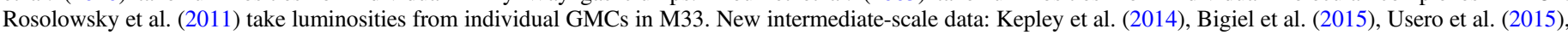

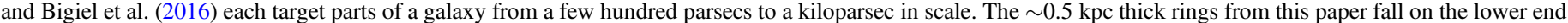
of the extragalactic scale-larger than individual GMCs but smaller than entire galaxies.

$\Sigma_{\text {dense }} / \Sigma_{\text {mol }}$, or $f_{\text {dense }}$. We plot results for our ALMA targets, NGC 5194, the Usero et al. (2015) pointings, and the Gao \& Solomon (2004) survey.

These data all show a similar picture: a correlation exists between $\Sigma_{\mathrm{SFR}} / \Sigma_{\text {mol }}$ and $\Sigma_{\text {dense }} / \Sigma_{\text {mol }}$ with the expected sense, that a higher dense gas fraction does imply a higher star formation efficiency. However, the scatter about this relation is large compared to the dynamic range in $\Sigma_{\text {dense }} / \Sigma_{\text {mol }}$ or $\Sigma_{\mathrm{SFR}} / \Sigma_{\text {mol }}$.

The weak correlation does not appear to be an artifact of limited dynamic range. We picked our targets to span a large range of $\Sigma_{\mathrm{SFR}} / \Sigma_{\mathrm{mol}}$, with the goal of testing the dense gas threshold hypothesis. Indeed, Figure 7 shows that our galaxies exhibit an order-of-magnitude spread in $\Sigma_{\text {dense }} / \Sigma_{\text {mol }}$. This is a large fraction of the total variation seen in the local universe (e.g., Saintonge et al. 2011; Leroy et al. 2013b, 2015). We also see roughly an order-of-magnitude variation in $\Sigma_{\text {dense }} / \Sigma_{\text {mol }}$ across our sample. If a sharp correlation between $\Sigma_{\mathrm{SFR}} / \Sigma_{\text {mol }}$ and $\Sigma_{\text {dense }} / \Sigma_{\text {mol }}$ was present, we would expect to see it in Figure 7.

The black line in the left panel of Figure 7 shows the expectation for a fixed SFR per unit dense gas. We set the slope to the median $\Sigma_{\text {dense }} / \Sigma_{\text {mol }}$ value of our sample and illustrate the $\pm 1 \sigma$ range as a shaded gray region. This median ratio, $\Sigma_{\mathrm{SFR}} / \Sigma_{\text {dense }}=1.10 \times 10^{-8}$ with 0.25 dex scatter, is significantly higher than that found by Gao \& Solomon (2004), $9.33 \times 10^{-10}$, but comparable to those found by Usero et al. (2015) and García-Burillo et al. (2012), $1.12 \times 10^{-8}$ and $1.45 \times 10^{-8}$, respectively. Recall that each of these works calculates SFR differently and that the Gao \& Solomon (2004)

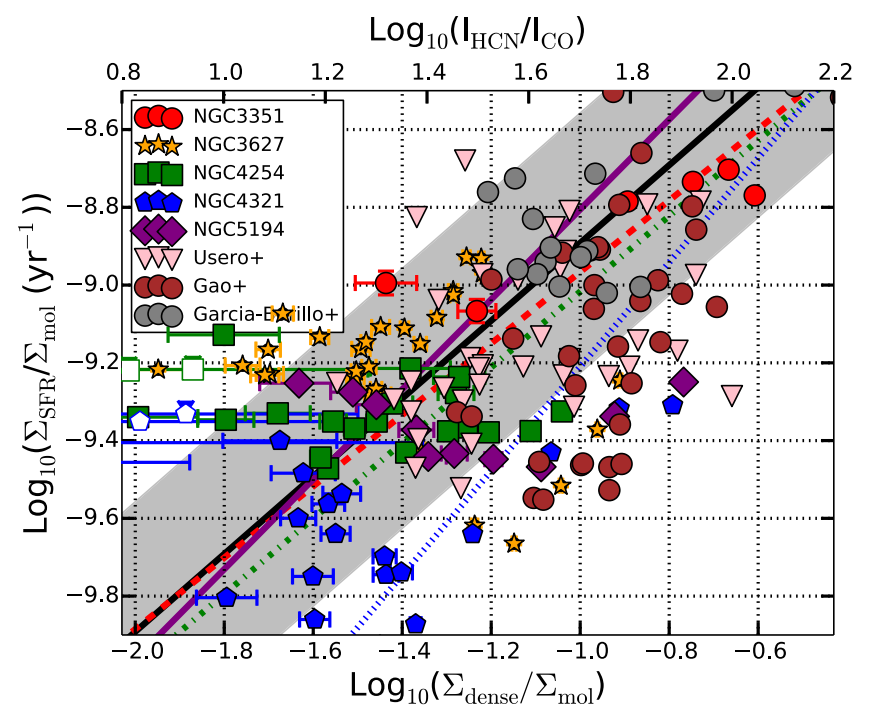

Figure 7. $\Sigma_{\mathrm{SFR}} / \Sigma_{\mathrm{mol}}$, or the normalized rate at which molecular gas forms stars, as a function of $\Sigma_{\text {dense }} / \Sigma_{\text {mol }}$, or the dense gas fraction as traced by $I_{\mathrm{HCN}} / I_{\mathrm{CO}}$. Higher dense gas fractions do correspond to more efficient star formation in the total molecular gas, but with large scatter, which we show below to be physical in nature. Lines show the median, best fit, and scatter for our survey along with the measurements of Gao \& Solomon (2004), GarcíaBurillo et al. (2012), and Usero et al. (2015). Statistically, the HCN/CO ratio is a relatively poor predictor of $\Sigma_{\mathrm{SFR}} / \mathrm{CO}$ when all data are considered together, though it performs better in some individual galaxies or subsamples.

and García-Burillo et al. (2012) samples focus on starburst systems, whereas our sample consists of more normal starforming galaxies. 
The red dashed line in Figure 7 shows an ordinary leastsquares bisector fit to our data. The best-fit power law, weighting all significant detections equally, is

$$
\log _{10} \frac{\Sigma_{\mathrm{SFR}}}{\Sigma_{\text {mol }}} \approx-8.05+0.92 \log _{10} \frac{\Sigma_{\text {dense }}}{\Sigma_{\text {mol }}} .
$$

This relation should be taken as indicative of the scaling between $\Sigma_{\mathrm{SFR}} / \Sigma_{\text {mol }}$ and $\Sigma_{\text {dense }} / \Sigma_{\text {mol }}$ in our data. As is visible from the plot, the choice of which data to fit (starbursts, whole galaxies, individual regions, etc.), the weighting applied to different data (e.g., by area, luminosity, or galaxy), and the model used (e.g., including astrophysical scatter or not) all have the potential to alter the fit substantially. This would not be true if $\Sigma_{\text {dense }} / \Sigma_{\text {mol }}$ perfectly predicted $\Sigma_{\mathrm{SFR}} / \Sigma_{\text {mol }}$ in a universal way across all systems.

The green, blue, and purple lines show similar fits applied to only the Usero et al. (2015), Gao \& Solomon (2004), and García-Burillo et al. (2012) data. Considering only the Usero et al. (2015) and García-Burillo et al. (2012) data, we fit slightly steeper slopes than for our data, but we find a consistent normalization near $\log _{10} f_{\text {dense }} \sim-1.5$. The fit to the Gao \& Solomon (2004) fit has a steeper slope and lower intercept than our data, consistent with the offset in measured $\Sigma_{\mathrm{SFR}} / \Sigma_{\text {dense }}$ mentioned above. The fits for both Usero et al. (2015) and García-Burillo et al. (2012) are consistent with the fit to our data within the uncertainties.

Thus, our best-fit slope of $\sim 1$ agrees with the schematic picture that $\Sigma_{\text {dense }} / \Sigma_{\text {mol }}$ as traced by $\Sigma_{\mathrm{HCN}} / \Sigma_{\mathrm{CO}}$ should predict $\Sigma_{\mathrm{SFR}} / \Sigma_{\mathrm{mol}}$ in a simple way. However, our results also show that $\Sigma_{\text {dense }} / \Sigma_{\text {mol }}$ is not a highly accurate predictor of $\Sigma_{\mathrm{SFR}} / \Sigma_{\mathrm{mol}}$. Across our whole sample, the rank correlation between $\Sigma_{\mathrm{SFR}} / \Sigma_{\text {mol }}$ and $\Sigma_{\text {dense }} / \Sigma_{\text {mol }}$ is only 0.13 . Meanwhile, the overall scatter in $\Sigma_{\mathrm{SFR}} / \Sigma_{\text {mol }}$ is 0.33 dex.

Note that individual galaxies do show tighter or looser individual trends in Figure 7, and we report the rank correlation coefficients and logarithmic fits for all of the scaling relations considered in this work in Tables $5-8 . I_{\mathrm{HCN}} / I_{\mathrm{CO}}$ can be a good predictor of $\Sigma_{\mathrm{SFR}} / \Sigma_{\mathrm{mol}}$ for some galaxies. But overall, $I_{\mathrm{HCN}} / I_{\mathrm{CO}}$ remains only a moderately good predictor of $\Sigma_{\mathrm{SFR}} / \Sigma_{\text {mol }}$. The relationship appears particularly poor for our radial profiles, which emphasize the contrast between nuclear regions and the surrounding disks.

Murphy et al. (2015) find similar results for NGC 3627. They find that the $\mathrm{SFE}_{\text {mol }}$ decreases as a function of $f_{\text {dense }}$ for the nucleus and two extranuclear star-forming regions in the disk of NGC 3627. Furthermore, they find that the velocity dispersion of the dense gas decreases with increasing $\mathrm{SFE}_{\mathrm{mol}}$. They conclude that the dynamical state of the dense gas, not its abundance, is what sets the SFR.

Certainly, stars form in the densest parts of local molecular clouds. Why is $I_{\mathrm{HCN}} / I_{\mathrm{CO}}$ only a weak predictor of the star formation efficiency of molecular gas? In the next two sections, we consider whether $I_{\mathrm{HCN}} / I_{\mathrm{CO}}$ indeed traces the dense gas mass fraction (or at least indicates the mean ISM density) and then present evidence for a context-dependent role for dense gas in star formation (building on García-Burillo et al. 2012; Usero et al. 2015; Bigiel et al. 2016).

\subsection{Is HCN-to-CO Ratio Tracing Density?}

Does the surprising lack of correlation between $\Sigma_{\mathrm{TIR}} / I_{\mathrm{CO}}$ and $I_{\mathrm{HCN}} / I_{\mathrm{CO}}$ reflect a problem with our interpretation of
Table 5

$\Sigma_{\mathrm{SFR}} / \Sigma_{\text {mol }}$ vs. $\Sigma_{\text {dense }} / \Sigma_{\text {mol }}$

\begin{tabular}{lrr}
\hline \hline Data Set & Rank Corr. & $\log _{10} \Sigma_{\text {SFR }} / \Sigma_{\text {dense }}$ \\
\hline Radial profiles & $0.77(0.052)$ & $-7.94( \pm 0.19)$ \\
NGC 3351 & $-0.01(0.488)$ & $-7.73( \pm 0.34)$ \\
NGC 3627 & $0.10(0.342)$ & $-7.89( \pm 0.26)$ \\
NGC 4254 & $0.21(0.202)$ & $-8.26( \pm 0.23)$ \\
NGC 4321 & $-0.26(0.236)$ & $-8.12( \pm 0.28)$ \\
NGC 5194 & $0.13(0.003)$ & $-7.89( \pm 0.33)$ \\
All profiles & $0.42(0.005)$ & $-7.95( \pm 0.25)$ \\
Usero et al. (2015) & $0.65(0.000)$ & $-9.03( \pm 0.24)$ \\
Gao \& Solomon (2004) & $0.35(0.075)$ & $-7.84( \pm 0.20)$ \\
García-Burillo et al. (2012) & $0.30(0.000)$ & $-7.96( \pm 0.31)$ \\
\hline All data & & \\
\hline
\end{tabular}

Note. Rank correlation quotes with $p$ value in parentheses; $\log _{10} \Sigma_{\mathrm{SFR}} / \Sigma_{\text {dense }}$ in units of $\log _{10} \mathrm{yr}^{-1}$. We quote the mean of the logarithm of the ratio and the $\pm 1 \sigma$ scatter in the log of the ratio. "All data" treats all data points with equal weight, regardless of the spatial scale sampled.

a Bigiel et al. (2016).

Table 6

$\Sigma_{\mathrm{SFR}} / \Sigma_{\text {dense }}$ vs. Other

\begin{tabular}{lcc}
\hline \hline Data Set & Rank Corr. (versus $\left.\Sigma_{*}\right)$ & Rank Corr. (versus $\left.\Sigma_{\text {mol }}\right)$ \\
\hline Radial profiles & $-1.0(0.002)$ & $-1.0(0.001)$ \\
NGC 3351 & $-0.74(0.000)$ & $-0.76(0.000)$ \\
NGC 3627 & $-0.69(0.001)$ & $-0.69(0.001)$ \\
NGC 4254 & $-0.90(0.000)$ & $-0.74(0.000)$ \\
NGC 4321 & $-1.0(0.000)$ & $-1.0(0.000)$ \\
NGC 5194 & $-0.71(0.000)$ & $-0.73(0.000)$ \\
All profiles & $-0.60(0.000)$ & $-0.55(0.000)$ \\
Usero et al. (2015) & $-0.66(0.000)$ & $-0.72(0.000)$ \\
\hline All data &
\end{tabular}

Note. Rank correlation quotes with $p$ value in parentheses. We quote the mean of the logarithm of the ratio and the $\pm 1 \sigma$ scatter in the log of the ratio. "All data" treats all data points with equal weight, regardless of the spatial scale sampled.

${ }^{\mathrm{a}}$ Bigiel et al. (2016).

$I_{\mathrm{HCN}} / I_{\mathrm{CO}}$ ? We take this ratio to trace the fraction of dense gas, $f_{\text {dense. }}$ In this picture, $\mathrm{CO}$ traces the bulk of the molecular gas, while $\mathrm{HCN}$ traces material with $n_{\mathrm{H} 2} \gtrsim 10^{4} \mathrm{~cm}^{-3}$ (e.g., Gao \& Solomon 2004; Krumholz \& Thompson 2007). This is the standard interpretation for this ratio, because the most effective density for HCN emission, $n_{\text {eff }}$, is much higher than both the $n_{\text {eff }}$ for $\mathrm{CO}$ and the mean density of a molecular cloud in the solar neighborhood. Meanwhile, $\mathrm{CO}$ traces the overall molecular gas mass, though not without caveats (see Bolatto et al. 2013).

Reality may be more complicated. We expect a wide distribution of densities in each beam, and the shape of that distribution can affect our interpretation of the line ratio. Variations in the optical depth, temperature, and chemical abundance of one or both species can also produce line ratio variations that mimic those expected from changing density. Although we lack a "smoking gun," our observations provide several indirect ways to test whether $I_{\mathrm{HCN}} / I_{\mathrm{CO}}$ indeed reflects the density distribution in our data.

First, in the left panel of Figure 8 we compare $I_{\mathrm{HCN}} / I_{\mathrm{CO}}$ to the average surface density on much larger scales, traced by the 

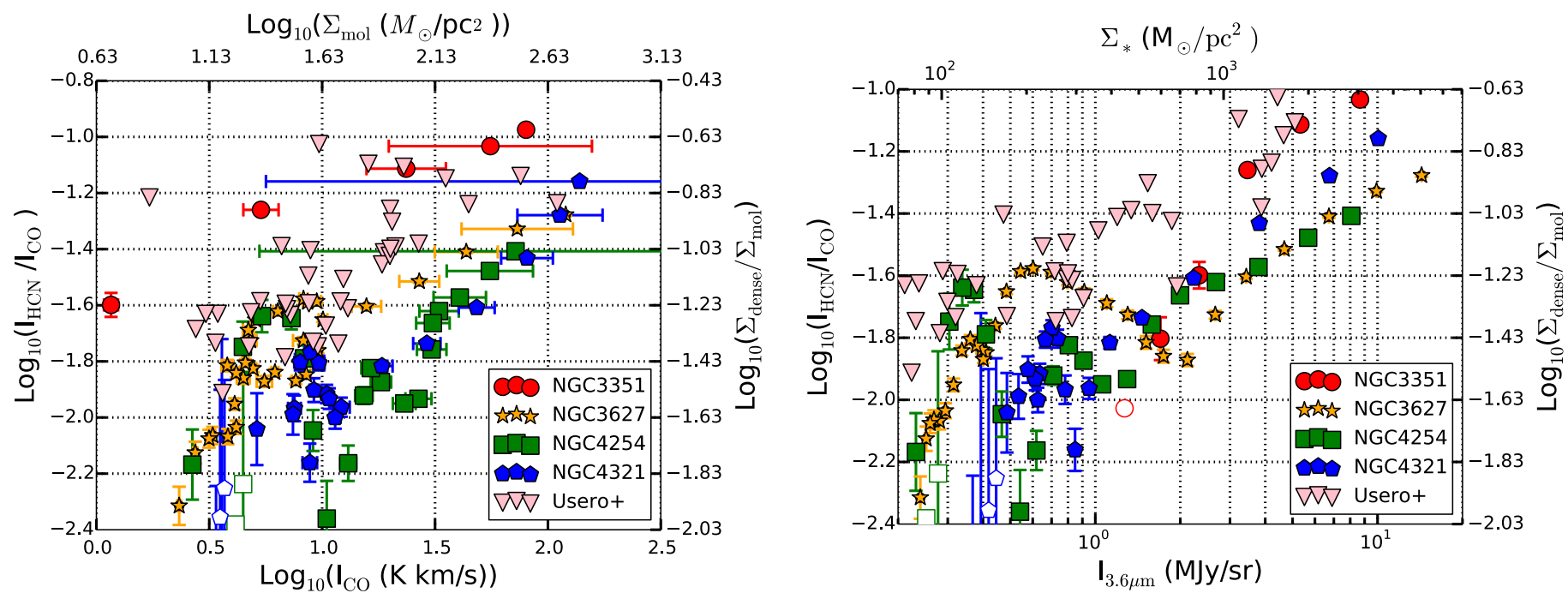

Figure 8. $\mathrm{HCN} / \mathrm{CO}$, tracing the dense gas fraction, as a function of observable cognates of molecular gas (left) and stellar surface density (right). The left panel shows $\mathrm{HCN} / \mathrm{CO}$ as a function of mean molecular gas surface density, estimated from $I_{\mathrm{CO}}$ using a fixed conversion factor. The right panel shows $\mathrm{HCN} / \mathrm{CO}$ as a function of stellar surface density, estimated from contaminant-corrected $3.6 \mu \mathrm{m}$ maps. In both cases, a higher overall surface density corresponds to a larger apparent dense gas fraction. The correlation with stellar surface density is particularly striking (as in Usero et al. 2015).

average surface brightness of $\mathrm{CO}$ in a ring. This large-scale surface density convolves the distribution of clouds and the internal density of each cloud, but it still provides a coarse measure of how dense the molecular ISM is in a given region of our Galaxy. The figure shows that $I_{\mathrm{HCN}} / I_{\mathrm{CO}}$ indeed correlates with $I_{\mathrm{CO}}$, suggesting that high dense gas fractions occur in regions of high mean density.

This correlation appears stronger within individual galaxies than taking all of our targets together (see Table 7). Conversion factor variations (Sandstrom et al. 2013) and differences in the sub-beam ISM structure (e.g., Leroy et al. 2016) likely contribute to these galaxy-to-galaxy variations. In that sense, our $8^{\prime \prime} \sim 500 \mathrm{pc}$ resolution still limits this test. A stronger version of this test will be to correlate $I_{\mathrm{HCN}} / I_{\mathrm{CO}}$ with cloudscale (tens of parsec) surface and volume density estimates based on high-resolution $\mathrm{CO}$ imaging. We will present such a comparison in M. Gallagher et al. (2018, in preparation).

Our line suite allows us to construct several ratios that constrain the shape of the density distribution and the potential impact of abundance variations. We show these in Figure 9. There we plot $I_{\mathrm{HCO}+} / I_{\mathrm{CO}}$ and $I_{13 \mathrm{CO}} / I_{\mathrm{CO}}$, each as a function of $I_{\mathrm{HCN}} / I_{\mathrm{CO}}$. These ratios offer a check that other dense gas tracers show the same results as $\mathrm{HCN}$ and offer some constraint on the sub-beam density distribution.

What do we see? $I_{\mathrm{HCN}} / I_{\mathrm{CO}}$ and $I_{\mathrm{HCO}+} / I_{\mathrm{CO}}$ correlate throughout our data, with a median ratio of $I_{\mathrm{HCO}+} / I_{\mathrm{HCN}}=$ 0.76 and a scatter of 0.24 . The solid black line illustrates a constant $I_{\mathrm{HCO}+} / I_{\mathrm{HCN}}$ equal to this median ratio, with the black dashed lines showing the scatter in our data. We would expect abundance variations unrelated to density to induce scatter in this relation. The observed correlation suggests that our results would be qualitatively similar if we used $I_{\mathrm{HCO}+} / I_{\mathrm{CO}}$ to trace dense gas instead of $I_{\mathrm{HCN}} / I_{\mathrm{CO}}$.

Correlations between the $I_{\mathrm{HCO}+} / I_{\mathrm{HCN}}$ ratio and environment have been studied in great detail in luminous infrared galaxies (LIRGs; e.g., Graciá-Carpio et al. 2006, 2008; Imanishi et al. 2006; Privon et al. 2015). These studies reveal some source-to-source variations. But consistent with our results here, starburst-dominated systems show a mean ratio
Table 7

$I_{\mathrm{HCN}} / I_{\mathrm{CO}}$ vs. Other

\begin{tabular}{lcc}
\hline \hline Data Set & Rank Corr. (versus $\Sigma_{*}$ ) & Rank Corr. (versus $\left.I_{\mathrm{CO}}\right)$ \\
\hline Radial profiles & $1.0(0.001)$ & $1.0(0.001)$ \\
NGC 3351 & $0.81(0.000)$ & $0.86(0.000)$ \\
NGC 3627 & $0.43(0.037)$ & $0.43(0.037)$ \\
NGC 4254 & $0.71(0.001)$ & $0.72(0.001)$ \\
NGC 4321 & $1.0(0.000)$ & $1.0(0.000)$ \\
NGC 5194 & $0.67(0.000)$ & $0.56(0.000)$ \\
All profiles & $0.78(0.000)$ & $0.72(0.000)$ \\
Usero et al. (2015) & $0.67(0.000)$ & $0.56(0.000)$
\end{tabular}

Note. Rank correlation quotes with $p$ value in parentheses. We quote the mean of the logarithm of the ratio and the $\pm 1 \sigma$ scatter in the log of the ratio. "All data" treats all data points with equal weight, regardless of the spatial scale sampled.

${ }^{\mathrm{a}}$ Bigiel et al. (2016).

$I_{\mathrm{HCO}+} / I_{\mathrm{HCN}} \approx 0.77 \pm 0.24$ (Privon et al. 2015) and an overall good correlation between $I_{\mathrm{HCO}+}$ and $I_{\mathrm{HCN}}$. The gray line and region in Figure 9 show this ratio and scatter from Privon et al. (2015).

$I_{\mathrm{HCO}+} / I_{\mathrm{CO}}$ variations appear modestly weaker than $I_{\mathrm{HCN}} / I_{\mathrm{CO}}$ variations. That is, the slope in the left panel of Figure 9 appears slightly sublinear. Meanwhile, the right panel shows that the magnitude of $I_{13 \mathrm{CO}} / I_{\mathrm{CO}}$ variations is much smaller than that in $I_{\mathrm{HCN}} / I_{\mathrm{CO}}$. As discussed in Leroy et al. (2017b), this is the expected behavior for a distribution of gas densities similar to a lognormal. The $n_{\text {eff }}$ of optically thin ${ }^{13} \mathrm{CO}$ is higher than that of $\mathrm{CO}$, which is usually significantly optically thick. But both $n_{\text {eff }}$ are lower than the mean density of the medium. Thus, the fractional change in the gas above these two densities will vary only weakly as the density distribution shifts. Meanwhile, $\mathrm{HCO}^{+}$has a slightly lower $n_{\text {eff }}$ than $\mathrm{HCN}$. For a curving distribution like a lognormal, we expect variations in $\mathrm{HCN}$ emission to be stronger than those in $\mathrm{HCO}^{+}$as the mean of the density distribution shifts (Leroy et al. 2017b). Conversely, the modest changes in $I_{13 \mathrm{CO}} / I_{\mathrm{CO}}$ 

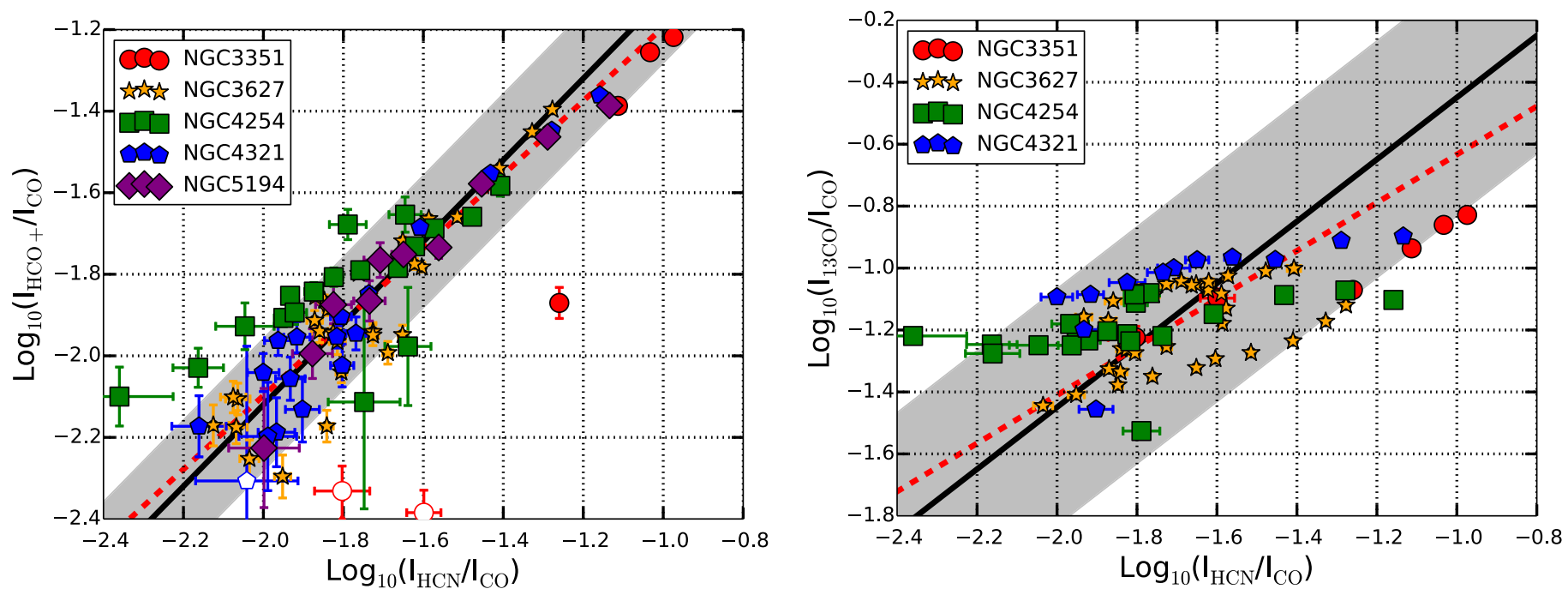

Figure 9. Line ratios for our radial profiles. Left: $I_{\mathrm{HCO}+} / I_{\mathrm{CO}}$ as a function of $I_{\mathrm{HCN}} / I_{\mathrm{CO}}$. The black line shows the median $\mathrm{HCO}^{+} / \mathrm{HCN}$ ratio and its $\pm 1 \sigma$ range in our data. The gray shaded region illustrates the $\pm 1 \sigma$ range found by Privon et al. (2015) for luminous infrared galaxies. The two line ratios track one another, reinforcing that our results are not specific to HCN. There is some indication of a slightly sublinear slope to the $I_{\mathrm{HCO}+} / I_{\mathrm{CO}} \mathrm{vs}$. $I_{\mathrm{HCN}} / I_{\mathrm{CO}}$ relation, which would be expected for a lognormal distribution given the lower critical density of $\mathrm{HCO}^{+}$. Right: ${ }^{13} \mathrm{CO} / \mathrm{CO}$ as a function of $I_{\mathrm{HCN}} / I_{\mathrm{CO}}$. The black line shows the median ${ }^{13} \mathrm{CO} / \mathrm{HCN}$ in our sample. The sublinear slope can be expected if the effective density for ${ }^{13} \mathrm{CO}$ emission lies below the median density by mass while HCN traces comparatively dense gas.

indicate that a power law or other self-similar distribution would be a poor description of the entire density distribution.

Thus, our data offer support for the idea that $I_{\mathrm{HCN}} / I_{\mathrm{CO}}$ traces the dense gas fraction: high $I_{\mathrm{HCN}} / I_{\mathrm{CO}}$ occurs in regions of high surface brightness, $I_{\mathrm{HCN}} / I_{\mathrm{CO}}$ tracks with $I_{\mathrm{HCO}+} / I_{\mathrm{CO}}$, and the sense of line ratio variations is that expected from a lognormallike distribution subresolution. However, variations in the quantitative translation of $I_{\mathrm{HCN}} / I_{\mathrm{CO}}$ to $f_{\text {dense }}$ remain likely. Temperature, abundance, and optical depth variations of both lines remain plausible and hard to quantify (e.g., JiménezDonaire et al. 2017a). These could affect both $\alpha_{\mathrm{HCN}}$ and $\alpha_{\mathrm{CO}}$. If both lines are optically thick, then temperature variations might be expected to cancel out to some degree, but this is far from certain. We proceed quoting results in terms of the observable $I_{\mathrm{HCN}} / I_{\mathrm{CO}}$ and noting the corresponding $f_{\text {dense }}$ for our fiducial $\alpha_{\mathrm{HCN}}$ and $\alpha_{\mathrm{CO}}$, but we note the latter as uncertain.

Wide-field mapping of local molecular clouds does indicate $\alpha_{\mathrm{HCN}}$ variations within, e.g., Orion (see Kauffmann et al. 2017; Pety et al. 2017). Usero et al. (2015) considered a wide range of possible $\alpha_{\mathrm{HCN}}$. They show that such variations are highly unlikely to conspire in a way that returns a fixed star formation efficiency of dense gas. Still, the quantitative translation of line ratios into density variations remains uncertain. More multiline wide-area maps of the Milky Way will be key in this regard. Meanwhile, synthetic multiline modeling of the full line suite (e.g., building on Leroy et al. 2017b), comparison to small-scale ISM structure measurements (M. Gallagher et al. 2018, in preparation), and improved constraints on optical depth (Jiménez-Donaire et al. 2017a) are next goals of this project and the EMPIRE survey.

\subsection{What Sets the Star Formation Efficiency of Dense Gas?}

Our data follow the broad correlation between SFR and HCN luminosity, but we see clear evidence of variations in $\Sigma_{\mathrm{SFR}} / \Sigma_{\text {dense, }}$, the apparent efficiency with which dense gas forms stars.

\subsubsection{Implied Depletion Time and Efficiency per Freefall Time}

For our fiducial conversion factors, our measured HCN/SFR ratios imply a median dense gas depletion time, $\tau_{\text {dep }}^{\text {dense }} \equiv$ $M_{\text {dense }} /$ SFR, of $\tau_{\text {dep }}^{\text {dense }} \sim 78 \mathrm{Myr}$. This value is lower than the median $\tau_{\text {dep }}^{\text {dense }} \sim 110 \mathrm{Myr}$ from Usero et al. (2015) and $\tau_{\text {dep }}^{\text {dense }} \sim 140$ Myr from Gao \& Solomon (2004).

Our CO/SFR ratios give a mean molecular gas depletion time, $\tau_{\text {dep }}^{\text {mol }} \equiv M_{\text {mol }} / \mathrm{SFR}$, of 2.0 Gyr. This median value is comparable to the median $\tau_{\text {dep }}^{\text {mol }}=1.7 \mathrm{Gyr}$ from Usero et al. (2015) and somewhat higher than the median $\tau_{\text {dep }}^{\text {mol }}=1.1 \mathrm{Gyr}$ from Gao \& Solomon (2004).

When comparing these numbers, note that the regions, galaxies, and spatial scales sampled differ among our work, the bright disk pointings of Usero et al. (2015), and the starburstheavy sample of Gao \& Solomon (2004). Also recall that our sample was constructed to capture variations in CO/SFR, not to be representative of the local galaxy population. With this in mind, these values agree reasonably well with one another and with the $\tau_{\text {dep }}^{\text {mol }}=1-2$ Gyr found from observations targeting large areas and many galaxies (e.g., Saintonge et al. 2011; Leroy et al. 2013b; Bolatto et al. 2017).

If we naively take all CO- and $\mathrm{HCN}$-emitting gas to lie at the effective critical density for the line, then we can estimate the fraction of total and dense gas converted into stars per freefall time, e.g., following Krumholz \& Tan (2007). We take the density of all molecular gas to be $n_{\mathrm{H} 2} \sim 10^{2} \mathrm{~cm}^{-3}$ $\left(\tau_{f f} \sim 3 \mathrm{Myr}\right)$ and the density of the dense gas producing $\mathrm{HCN}$ emission to be $n_{\mathrm{H} 2} \sim 10^{5} \mathrm{~cm}^{-3}\left(\tau_{f f} \sim 0.1 \mathrm{Myr}\right)$. Then both $\mathrm{HCN}$ and $\mathrm{CO}$ suggest an efficiency per freefall time of $\sim 0.2 \%-0.3 \%$. This value agrees well with direct estimates of the efficiency per freefall time based on high-resolution imaging in NGC 5194 (Leroy et al. 2017a) and on previous estimates based on dense gas spectroscopy (García-Burillo et al. 2012; Usero et al. 2015). 

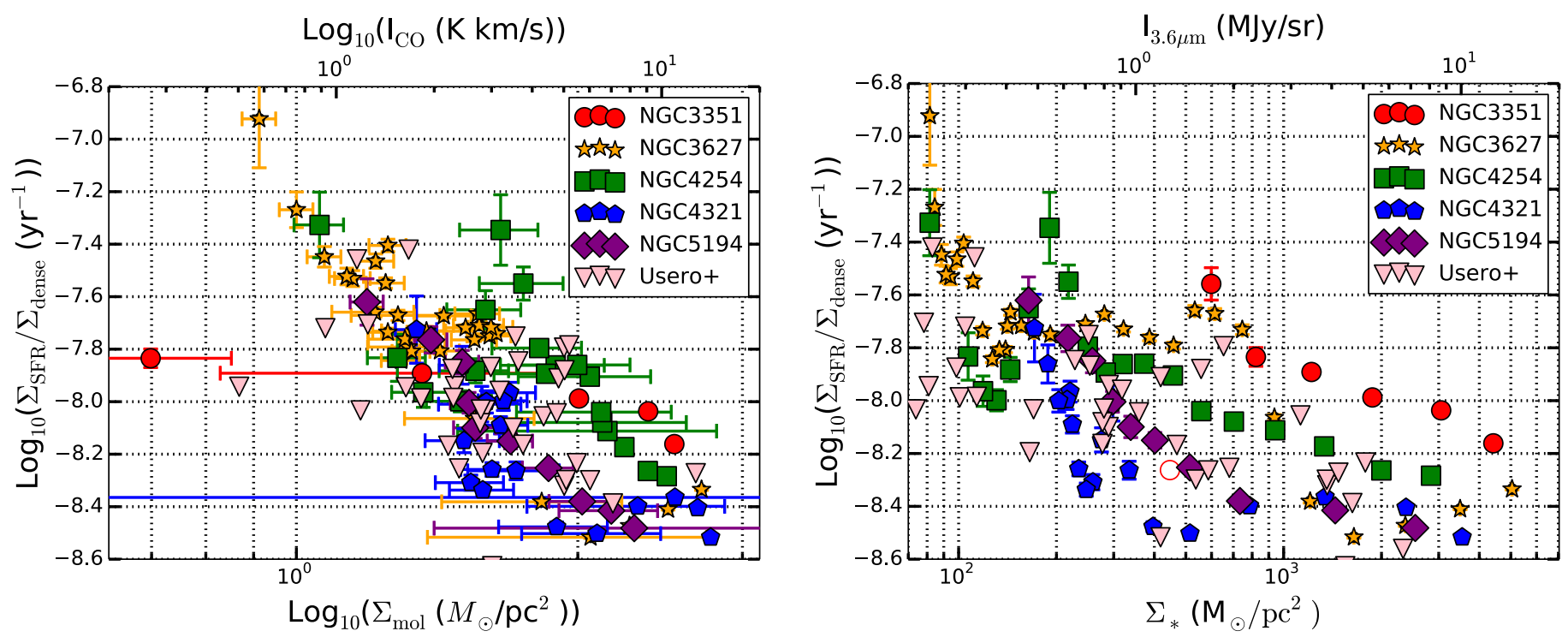

Figure 10. $\Sigma_{\mathrm{SFR}} / \Sigma_{\text {dense }}$, or the star formation efficiency of dense gas, as a function of tracers of the molecular gas (left) and stellar (right) surface density. $\Sigma_{\mathrm{SFR}} / \Sigma_{\mathrm{dense}}$ anticorrelates with $\Sigma_{\mathrm{mol}}$, traced by $I_{\mathrm{CO}}$, and with $\Sigma_{*}$, as traced by contaminant-corrected $3.6 \mu \mathrm{m}$ intensity. Thus, dense gas appears less efficient at star formation at high surface densities. The primary axes adopt our fiducial translations to physical quantities and report the molecular gas or stellar surface density $(x)$ and the star formation efficiency of dense gas $\left(\mathrm{SFR} / M_{\text {dense }}\right)$. The alternate axes present the observational data.

\subsubsection{Variation of Dense Gas Star Formation Efficiency with Environment}

Usero et al. (2015) and Bigiel et al. (2016) showed that stellar surface density $\left(\Sigma_{*}\right)$ and the molecular-to-atomic-gas ratio act as "third parameters" in the relationship between $f_{\text {dense }}$ and $\mathrm{SFE}_{\text {dense. }}$. In their studies, regions with high stellar surface density or high molecular gas fraction also have high dense gas fractions. However, in these same high stellar surface density regions dense gas appears less efficient at forming stars.

Such behavior might be expected if clouds with a high mean density $\left(n_{0}\right)$ still only form stars in local overdensities within the cloud. This would be true if these clouds have a typical, roughly virialized, dynamical state at large scales despite their high $n_{0}$. Then, where $n_{0}$ is high, the immediately star-forming gas engaged in direct collapse and star formation may lie at still higher densities. Similar arguments have been proposed to explain the behavior of our own Galactic center (e.g., Kauffmann et al. 2013; Kruijssen et al. 2014; Rathborne et al. 2015).

In this scenario, $\mathrm{SFE}_{\text {dense }}$ for "dense" gas defined by some fixed density threshold will anticorrelate with $n_{0}$. Thus, e.g., we would expect $\Sigma_{\mathrm{SFR}} / I_{\mathrm{HCN}}$ to go down as the mean density of the ISM goes up and HCN traces more "normal" and less directly star-forming gas. In this case $\mathrm{SFE}_{\text {dense }}$ should anticorrelate with environmental quantities related to the mean density of the ISM.

To explore this, we plot $\Sigma_{\mathrm{SFR}} / \Sigma_{\text {dense, }}$, where $\Sigma_{\text {dense }}$ is a scaled $I_{\mathrm{HCN}}$, or $\mathrm{SFE}_{\mathrm{HCN}}$, as a function of the surface densities of stars and $I_{\mathrm{CO}}$, tracing molecular gas surface density. Figure 10 shows that $\Sigma_{\mathrm{SFR}} / \Sigma_{\text {dense }}$ indeed decreases with increasing $\Sigma_{\text {mol }}$. A reasonable fit to the relationship is

$$
\log _{10} \frac{\Sigma_{\text {SFR }}}{\Sigma_{\text {dense }}}=-6.72-0.72 \log _{10} \Sigma_{\text {mol }} .
$$

Here $\Sigma_{\text {mol }}$ in our sample is the azimuthally averaged intensity of $\mathrm{CO}$ emission multiplied by $\alpha_{\mathrm{CO}}$. This traces molecular gas surface density on large scales (recall that our resolution is already hundreds of parsecs). A more rigorous test of this idea using higher-resolution imaging will be presented in M. Gallagher et al. (2018, in preparation).

Stellar surface densities exceed the gas surface density over the whole area of our survey, and the stars play a key role in setting the gravitational potential within which the gas exists. If the gas is in some semblance of vertical dynamic equilibrium, then one expects high $\Sigma_{*}$ to also lead to higher average midplane pressure and so a higher average gas density. The right panel of Figure 10 shows $\Sigma_{\mathrm{SFR}} / \Sigma_{\text {dense }}$ as a function of $\Sigma_{*}$. As in Usero et al. (2015) and Bigiel et al. (2016), we observe a clear anticorrelation between $\Sigma_{\mathrm{SFR}} / \Sigma_{\text {dense }}$ and $\Sigma_{*}$, so that in regions with high stellar surface density dense gas appears worse at forming stars.

$\Sigma_{\mathrm{SFR}} / \Sigma_{\text {dense }}$ and $\Sigma_{*}$ have a rank correlation coefficient of -0.71 , indicating a stronger relationship than between $\Sigma_{\mathrm{SFR}} / \Sigma_{\text {dense }}$ and $\Sigma_{\text {dense }} / \Sigma_{\text {mol }}$. Using the bisector method, we fit a power law of

$$
\log _{10} \frac{\Sigma_{\text {SFR }}}{\Sigma_{\text {dense }}}=-6.08-0.72 \log _{10} \Sigma_{*} .
$$

Both Equations (11) and (12) should be taken as indicative. We use all of the data available to us, but note that our sample is in no way statistically representative of the whole galaxy population.

Both panels in Figure 10 support the hypothesis that in a high mean density ISM, the gas that emits HCN is less effective at forming stars than in regions of low mean density. Caveats related to the ability of $\mathrm{HCN}$ to trace dense gas apply; we cannot rule out that instead this gas may simply be much better at emitting HCN. However, as discussed in Section 3.3, indirect evidence in our data set, as well as the discussion in Usero et al. (2015), gives us some confidence that we do capture density effects. And as discussed by Usero et al. (2015), HCN emission has so far been widely used to trace dense gas in the extragalactic literature (Gao \& Solomon 2004). These results complicate any threshold-style interpretation based on those observations. 
Table 8

Other vs. Pressure

\begin{tabular}{lcc}
\hline \hline Data Set & $\begin{array}{c}\text { Rank Corr. } \\
\left(\Sigma_{\text {SFR }} / \Sigma_{\text {dense }} \text { versus }\right)\end{array}$ & $\begin{array}{c}\text { Rank Corr. } \\
\left(\Sigma_{\text {dense }} / \Sigma_{\text {mol }} \text { versus }\right)\end{array}$ \\
\hline Radial profiles & & \\
NGC 3351 & $-1.0(0.001)$ & $1.0(0.001)$ \\
NGC 3627 & $-0.65(0.000)$ & $0.79(0.000)$ \\
NGC 4254 & $-0.69(0.001)$ & $0.43(0.037)$ \\
NGC 4321 & $-0.70(0.001)$ & $0.67(0.002)$ \\
NGC 5194 & $-1.0(0.000)$ & $1.0(0.000)$ \\
All profiles & $-0.71(0.000)$ & $0.57(0.000)$ \\
Usero et al. (2015) & $-0.47(0.001)$ & $0.67(0.000)$ \\
\hline All data & $-0.54(0.000)$ & $0.36(0.000)$ \\
\hline
\end{tabular}

Note. Rank correlation coefficients with $p$ value in parentheses. We quote the mean of the logarithm of the ratio and the $\pm 1 \sigma$ scatter in the log of the ratio. "All data" treats all data points with equal weight, regardless of the spatial scale sampled.

${ }^{a}$ Bigiel et al. (2016).

We focus on $I_{\mathrm{CO}} \sim \Sigma_{\mathrm{mol}}$ and $\Sigma_{*}$ because they relate to the mean density of the gas. Assuming that the weight of the gas is balanced by its random motions, this relationship may be captured by considering the dynamical equilibrium pressure, $P_{\mathrm{tot}} \sim P_{\mathrm{DE}} . P_{\mathrm{DE}}$ will depend on both $\Sigma_{*}$ and $\Sigma_{\mathrm{mol}}$ and may in turn relate to the mean density of a cloud, $n_{0}$. We return to this point in Section 3.6.

\subsection{What Sets the Dense Gas Fraction?}

We also observe $I_{\mathrm{HCN}} / I_{\mathrm{CO}}$, our observational tracer of $f_{\text {dense, }}$, to vary significantly across our sample. Adopting our fiducial conversion factors, our observed $I_{\mathrm{HCN}} / I_{\mathrm{CO}}$ implies $f_{\text {dense }}$ between $\sim 0.5 \%$ and $\sim 25 \%$ with a median $f_{\text {dense }} \sim 4 \%$.

This median value is lower than the median value of $f_{\text {dense }} \sim 8 \%$ from Usero et al. (2015) and the median value of $f_{\text {dense }} \sim 12 \%$ from Gao \& Solomon (2004). This deviation could be expected based on the construction of samples, as $f_{\text {dense }}$ is not expected to remain constant. Gao \& Solomon (2004) study gas-rich starburst galaxies. Meanwhile, the pointings of Usero et al. (2015) were chosen to be bright in $\mathrm{CO}$ and hence rich in gas. We target a wider area with less of a selection bias and thus recover lower dense gas fractions. Still wider area maps covering whole galaxy disks may return even lower median $f_{\text {dense }}$ (a main goal of EMPIRE; see M. Jimenez Donaire et al. 2018, in preparation).

Our median $f_{\text {dense }} \sim 4 \%$ is lower than the typical $f_{\text {dense }} \sim 10 \%$ found for local clouds by Lada et al. (2012). This may reflect different ways of tracing dense gas, as they use extinction rather than $\mathrm{HCN}$ emission and adopt a different nominal density. It may also reflect more sensitivity of our observations to an extended molecular component.

Following the logic laid out in the previous section, we would expect the $I_{\mathrm{HCN}} / I_{\mathrm{CO}}$, tracing $f_{\text {dense }}$, to correlate with both $\Sigma_{*}$ and $I_{\mathrm{CO}}$, tracing $\Sigma_{\mathrm{mol}}$. In Figure 8 , we plot $I_{\mathrm{HCN}} / I_{\mathrm{CO}}$ as a function of both quantities. The figure shows that $I_{\mathrm{HCN}} / I_{\mathrm{CO}}$ indeed increases with increasing $I_{\mathrm{CO}}$. Using the bisector method, we find a power-law fit for $I_{\mathrm{HCN}} / I_{\mathrm{CO}}$ versus $I_{\mathrm{CO}}$ of

$$
\log _{10} \frac{\mathrm{HCN}}{\mathrm{CO}}=-2.42+0.72 \log _{10} I_{\mathrm{CO}}
$$

Again, we emphasize that Equation (13) is specific to our data and that $I_{\mathrm{CO}}$ traces the molecular gas surface density averaged on moderately large (few hundred parsec to roughly kiloparsec) scales.

We also expect to find a higher mean density and hence perhaps more dense gas where the gravitational potential well is deeper. We compare $I_{\mathrm{HCN}} / I_{\mathrm{CO}}$ to $\Sigma_{*}$ in the right panel of Figure 8 . We observe a clear correlation between $I_{\mathrm{HCN}} / I_{\mathrm{CO}}$ and $\Sigma_{*}$. This has the sense that in regions with high stellar surface density there is more gas dense enough to produce $\mathrm{HCN}$ emission. $I_{\mathrm{HCN}} / I_{\mathrm{CO}}$ and $\Sigma_{*}$ exhibit a rank correlation coefficient of 0.74 , indicating a stronger relationship between $f_{\text {dense }}$ and $\Sigma_{*}$ than between $\Sigma_{\mathrm{TIR}} / I_{\mathrm{CO}}$ and $I_{\mathrm{HCN}} / I_{\mathrm{CO}}$.

A reasonable fit to our data, including NGC 5194 and the data of Usero et al. (2015), is

$$
\log _{10} \frac{\mathrm{HCN}}{\mathrm{CO}}=-3.28+0.62 \log _{10} \Sigma_{*} .
$$

This is moderately steeper than the relation found by Usero et al. (2015):

$$
\log _{10} \frac{\mathrm{HCN}}{\mathrm{CO}} \text { Usero }=-2.58+0.42 \log _{10} \Sigma_{*} .
$$

Thus, following Usero et al. (2015) and Bigiel et al. (2016), but now for maps of the disk and central regions of five galaxies, our observations reveal strong correlations between disk structure and $I_{\mathrm{HCN}} / I_{\mathrm{CO}}$. These correlations extend over roughly an order of magnitude in $I_{\mathrm{HCN}} / I_{\mathrm{CO}}$ and span two orders of magnitude in stellar surface density and $I_{\mathrm{CO}}$. We interpret this as evidence for a strong link between the dense gas fraction and local disk structure.

\subsection{Dynamical Equilibrium Pressure, Dense Gas, and Star Formation}

The large-scale gas surface density, traced by $I_{\mathrm{CO}}$, and the stellar surface density, $\Sigma_{*}$, appear to affect both $\mathrm{SFE}_{\text {dense }}$ and $f_{\text {dense }}$. Above, we hypothesized that these relationships emerge because the mean density of molecular clouds reflects their large-scale environment. Specifically, following Elmegreen (1989), Ostriker et al. (2010), Kim et al. (2011), and others, we noted that the state of the ISM should self-regulate so that the pressure averaged over some relatively large time and length scales will approach the "dynamical equilibrium" value. In this case, molecular clouds will represent overpressured regions against a background pressure that reflects the environment. Helfer \& Blitz (1997) argued for a version of this idea to explain the variable dense gas fractions observed in nearby galaxies, and both Usero et al. (2015) and Bigiel et al. (2016) noted this as one plausible family of explanations for their observations. Galactic center studies have considered similar scenarios.

To explore the role of pressure directly, we estimate the dynamical equilibrium pressure, $P_{\mathrm{DE}}$, for each ring in our sample (see Section 2.8). In Figure 11, we plot $\Sigma_{\text {dense }} / \Sigma_{\text {mol }}\left(\propto I_{\mathrm{HCN}} / I_{\mathrm{CO}}\right)$ and $\Sigma_{\mathrm{SFR}} / \Sigma_{\text {dense }}\left(\propto \Sigma_{\mathrm{SFR}} / I_{\mathrm{HCN}}\right)$ as a function of $P_{\mathrm{DE}}$.

A reasonable fit relating $P_{\mathrm{DE}}$ and $\Sigma_{\text {dense }} / \Sigma_{\mathrm{mol}}$ is

$$
\log _{10} \frac{\Sigma_{\text {dense }}}{\Sigma_{\text {mol }}}=-4.01+0.48 \log _{10} P_{\mathrm{DE}} .
$$



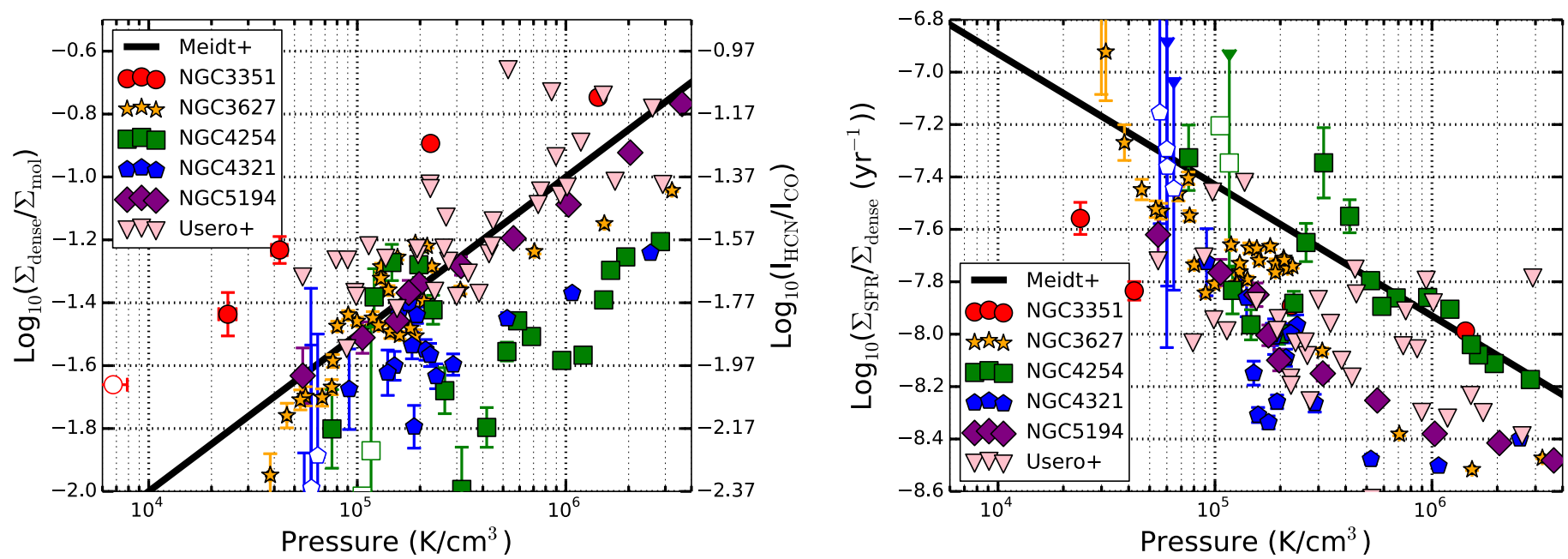

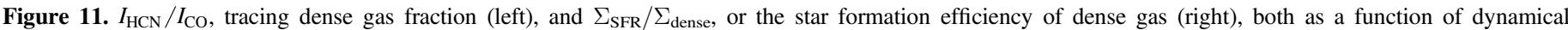

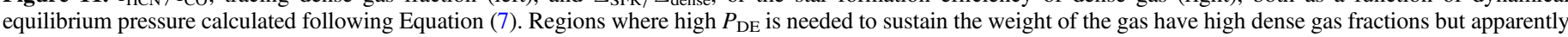

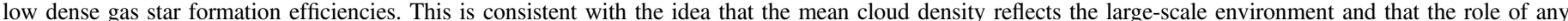

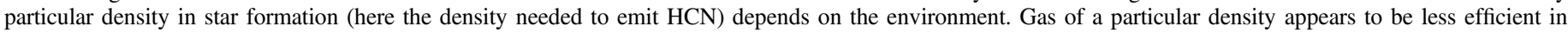
regions of high mean density. The black lines are model predictions from Meidt (2016).

$P_{\mathrm{DE}}$ and $\Sigma_{\mathrm{dense}} / \Sigma_{\mathrm{mol}}$ relate via a rank correlation coefficient of 0.60 . The sense of this correlation, as expected, is that regions with high $P_{\mathrm{DE}}$ exhibit high apparent dense gas fractions. This is the same as the sense of the correlations between $I_{\mathrm{CO}}$ and $I_{\mathrm{HCN}} / I_{\mathrm{CO}}$ and between $\Sigma_{*}$ and $I_{\mathrm{HCN}} / I_{\mathrm{CO}}$. The strength of the correlations does not recommend one of these as more fundamental, but we note that the correlation with $P_{\mathrm{DE}}$ has a physical explanation that would be expected to yield the other two.

High $P_{\mathrm{DE}}$ corresponds to a higher dense gas fraction, but also a lower star formation efficiency in the dense gas. In our data, a bisector fit between $P_{\mathrm{DE}}$ and $\Sigma_{\mathrm{SFR}} / \Sigma_{\text {dense }}$ yields

$$
\log _{10} \frac{\Sigma_{\mathrm{SFR}}}{\Sigma_{\text {dense }}}=-5.15-0.50 \log _{10} P_{\mathrm{DE}}
$$

The two quantities show a rank correlation coefficient of -0.71 , indicating a reasonably strong anticorrelation. Again, this goes in the same direction as the anticorrelation between $\Sigma_{*}$ and $\Sigma_{\mathrm{SFR}} / \Sigma_{\text {dense }}$ and between $I_{\mathrm{CO}}$ and $\Sigma_{\mathrm{SFR}} / \Sigma_{\text {dense }}$. In detail, the slopes differ, with somewhat shallower slopes for the $P_{\mathrm{DE}}$ relations than those treating $\Sigma_{*}$ or $I_{\mathrm{CO}}$ as the independent variable.

Should $P_{D E}$ affect the mean cloud density? Almost all regions that we consider have $P_{\mathrm{DE}} / k_{B} \gtrsim 10^{5} \mathrm{~cm}^{-3} \mathrm{~K}$. We expect that the average pressure of the ISM must approach these values. Compare this to the typical internal pressure for a local molecular cloud with $\Sigma_{\text {mol }} \sim 100 M_{\odot} \mathrm{pc}^{-2}$. Taking $P_{\text {int }} \sim G \pi \Sigma_{\text {mol }}^{2} / 2$, we see $P_{\text {in }} / k_{B} \sim 3 \times 10^{5} \mathrm{~cm}^{-3} \mathrm{~K}$. In the dynamical equilibrium picture, we would expect the internal pressure of molecular clouds to be somewhat higher than $P_{\mathrm{DE}}$, as molecular clouds represent overpressured regions in a dynamical ISM. In this sense, almost all of our regions have $P_{\mathrm{DE}}$ high enough that $P_{\text {int }}$ cannot remain fixed for clouds across our survey while still balancing the weight of the gas in the stellar potential.

Our result for the dense gas fraction agrees well with the prediction by Meidt (2016), shown by the black line in Figure 11. Meidt considers isothermal clouds bounded by interstellar pressure with $\rho \sim r^{-2}$ density profiles. We show her predicted $f_{\text {dense }}=\Sigma_{t, \mathrm{HCN}} / \Sigma_{t, \mathrm{~mol}}$. Here $\Sigma_{t, \mathrm{HNC}}$ is the column density above the critical density of $\mathrm{HCN}$ and $\Sigma_{t, \mathrm{~mol}}$ is the column density of the molecular material at the interface with the ambient ISM and is characterized by the ISM pressure. In this view, our calculated dynamical equilibrium pressure is interpreted as an ambient interstellar pressure; this assumption should work as long as the cloud cores modeled by Meidt (2016) do not make up too much of the mass. Figure 11 shows good agreement with most of our data and with the data of Usero et al. (2015). The corresponding prediction for $\mathrm{SFE}_{\mathrm{dense}}$ also captures our measured slope and intercept reasonably well.

Is there a threshold pressure for environment to affect dense gas? Conversely, our data do not yield much insight into the question whether the apparent dense gas fraction and $\mathrm{SFE}_{\text {dense }}$ continue to correlate with $P_{\mathrm{DE}}$ at the lower values of $P_{\mathrm{DE}}$ expected over most of the area in galaxy disks. One scenario would be that when $P_{\mathrm{DE}}$ falls far below $P_{\text {int }}$ of a typical molecular cloud and HI dominates the ISM, the clouds decouple and converge to a fairly universal population. In this case, the threshold picture of Lada et al. (2012) might still hold for analogs of the solar neighborhood.

Our study, which is optimized to study the contrast between inner molecular disks and the bright central regions of our targets, does not test this scenario. The full EMPIRE survey (M. Jimenez Donaire et al. 2018, in preparation) and full-disk, sensitive ALMA follow-up of our targets should offer more insight.

\section{Discussion}

We observed the inner few kiloparsecs of four star-forming galaxies, which show a range of apparent molecular gas depletion times, traced by $\mathrm{CO} / \mathrm{SFR}$. We analyze these data in conjunction with the first full-galaxy map from EMPIRE (Bigiel et al. 2016) and the pointed observations targeting $~ 30$ galaxy disks from Usero et al. (2015). Extending Usero et al. (2015) and Bigiel et al. (2016) and following García-Burillo et al. (2012), these data provide strong evidence that the ratios $I_{\mathrm{HCN}} / I_{\mathrm{CO}}$ and $\Sigma_{\mathrm{SFR}} / I_{\mathrm{HCN}}$ depend systematically on galactic 
environment. Assuming that these trace the dense gas fraction and the star formation efficiency of dense gas, this provides strong evidence for a dependence of dense gas fraction on environment and a context-dependent role for density in star formation.

With maps at ALMA's resolution, we are able to distinguish the relative structure of SFR, HCN emission, and CO emission. Our results suggest strong changes in the ratios among these quantities between the dense, central regions of some galaxies and the lower surface density disk regions. In our sample, this contrast appears strongest for strongly barred galaxies (NGC 3351, NGC 3627, and NGC 4321), though NGC 5194 (M51) shows some of the same signal despite the lack of a strong bar. A logical scenario to explain this behavior is that the bar drives gas to the center of the galaxy, creating bright, dense concentrations of molecular gas (e.g., Sheth et al. 2005; Krumholz \& Kruijssen 2015). In these regions, dense gas is common, but the star formation per unit dense gas appears low. NGC 5194 may exhibit a similar phenomenon thanks in part to the role of the arms in enabling gas streaming toward the center of the galaxy (see, e.g., Meidt et al. 2013; Querejeta et al. 2016).

This behavior is reminiscent of that seen in our own Galaxy. The Milky Way also has a strong bar, a massive concentration of dense molecular gas in the inner few hundred parsecs, and an apparently low ratio of star formation to dense gas compared to local clouds. Longmore et al. (2013) showed that in the Galactic center the dense gas integrated intensity is $\sim 100$ times that of non-Galactic center regions and that the star formation efficiency in the Galactic center is an order of magnitude lower than predicted.

We interpret our results as strong evidence that the behavior seen in the Milky Way is a general feature of strongly barred galaxies, with (at least apparent) central concentrations of dense gas but comparatively low (apparent) SFR per unit dense gas. More statistics are needed, especially to constrain any possible duty cycle (Krumholz \& Kruijssen 2015) and to understand how often such phenomena also occur in unbarred galaxies. But it seems clear that the Milky Way is far from a unique case in this regard.

\subsection{A Context-dependent Role for Density}

Our main observational result is that $\Sigma_{\text {dense }} / \Sigma_{\text {mol }}$ and $\Sigma_{\mathrm{SFR}} / \Sigma_{\text {dense }}$ show correlations with environment that are stronger than the correlation between $\Sigma_{\mathrm{SFR}} / \Sigma_{\text {mol }}$ and $\Sigma_{\text {dense }} / \Sigma_{\text {mol }}$. A natural interpretation for this is that the density distribution is a strong function of environment while the role of any particular density in star formation is context dependent.

Environment and Density: We find that the fraction of dense gas depends on environment. In this paper, $f_{\text {dense }}$ is traced mainly by $I_{\mathrm{HCN}} / I_{\mathrm{CO}}$, but $\mathrm{HCO}^{+}$and $\mathrm{CS}$ show similar results. Here "environment" means that $f_{\text {dense }}$ appears to increase as $\Sigma_{*}$ and $I_{\text {mol }} \sim \Sigma_{\text {mol }}$ increase.

Stellar and gas surface densities both relate to the mean pressure, $P_{\mathrm{DE}}$, needed for the ISM to support its own weight in the (mostly stellar) potential well. Inferring $P_{\mathrm{DE}}$ from observables, we show that $f_{\text {dense }}$ does correlate with $P_{\mathrm{DE}}$. As $P_{\mathrm{DE}}$ increases, $f_{\text {dense }}$ appears to increase. This follows closely on the results of Helfer \& Blitz (1997) that gas becomes denser in a deeper gravitational potential well where the ISM pressure is higher.
We suggest the following sketch to explain this correlation. In order for approximate equilibrium to hold, a disk must selfregulate to have $\sim P_{\mathrm{DE}}$ over a moderate length or timescale (Ostriker et al. 2010; Kim et al. 2011, 2013; Ostriker \& Shetty 2011; Kim \& Ostriker 2015). This dynamical equilibrium captures an average behavior. Molecular clouds will represent overdensities relative to this average. $P_{\mathrm{DE}}$ over large parts of our sample already corresponds to the typical internal pressure of a standard local cloud, $P_{\text {int }} / k_{B} \sim \pi G \Sigma_{\text {gas }}^{2} / 2 k_{B} \sim 3 \times$ $10^{5} \mathrm{~cm}^{-3} \mathrm{~K}$. For molecular clouds to represent overpressured regions (relative to the time and space average) in our data, their internal pressure must vary, increasing with $P_{\mathrm{DE}}$.

Our view is that the mean internal pressure of molecular clouds tracks $P_{\mathrm{DE}}$, and that as $P_{\mathrm{DE}}$ shifts so does the density distribution. That is, in high-pressure regions, the mean density is higher and the overall density distribution also shifts to higher values. We would also expect these regions to have higher line widths, and likely higher Mach numbers, which could also yield a larger dense gas fraction (see, e.g., Padoan \& Nordlund 2002; Krumholz \& Thompson 2007).

Our own observations, along with those of Usero et al. (2015) and Bigiel et al. (2016), support a scenario where the mean density of molecular clouds varies with the surface density of the disk. Viewing such a scenario through the lens of self-regulation to some mean $P_{\mathrm{DE}}$ based on disk structure and gas content seems reasonable. This explanation is not unique, but it does offer a logical scenario to explain what we see.

Mean Density, Dense Gas Fraction, and Star Formation: While $I_{\mathrm{HCN}} / I_{\mathrm{CO}}$ increases with $\Sigma_{*}, I_{\mathrm{CO}} \sim \Sigma_{\mathrm{mol}}$, and $P_{\mathrm{DE}}$, $\Sigma_{\mathrm{SFR}} / I_{\mathrm{HCN}}$ decreases. That is, as the dense gas fraction, and so likely the mean density, increases, the apparent rate of star formation per unit dense gas decreases. Taken at face value, this implies that any particular density-here specific $n_{\mathrm{eff}}$ of $\mathrm{HCN}$ - has a context-dependent role in star formation.

A context-dependent role for density in star formation could be expected if clouds are always marginally bound or virialized but vary in their mean density. Following, e.g., Krumholz \& Kruijssen (2015) and many others, overdensities will collapse out of the turbulent cascade and form stars. This immediately star-forming material seems likely to exhibit a power-law distribution of densities (see, e.g., Kainulainen et al. 2009; Federrath \& Klessen 2013). In clouds with high mean density, the immediate condition for these star-forming structures may be shifted to higher densities (e.g., Kruijssen et al. 2014). That is, stars should form out of overdensities in turbulent molecular clouds.

If $\mathrm{HCN}$ always traces the same gas density, $n_{\mathrm{HCN}}$, and if that density does not always capture the collapsing, power-law tail of the density distribution, then we could expect that the ratio of star formation per unit $\mathrm{HCN}$ will change as the density distribution changes. In regions of low $P_{\mathrm{DE}}$ and low mean cloud density, HCN traces high-density gas more immediately related to star formation. In high- $P_{\mathrm{DE}}$ regions with high mean density, HCN may trace a large fraction of the bulk mass in the cloud.

As above, this is not a unique interpretation. The mapping between $\mathrm{HCN}$ emission and dense gas may change with environment. And this interpretation requires that $\mathrm{HCN}$ not always sample a self-similar power law tail of the density distribution that behaves the same in any environment (see, e.g., Leroy et al. 2017b). There is support for a scenario like what we describe from the Milky Way center (Kepley 
et al. 2014; Rathborne et al. 2015), but much more work remains to be done.

Turbulence or Pressure? We have emphasized mean density in this section, but note that the Mach number also plays a key role in the density distribution for a turbulent cloud (Padoan \& Nordlund 2002; Krumholz \& McKee 2005). Usero et al. (2015) showed that, using the model of Krumholz \& Thompson (2007), plausible variations in the Mach number could explain many of their observations, along with those of García-Burillo et al. (2012) and Gao \& Solomon (2004). Observations suggest that as the mean density changes, the turbulent velocity dispersion will also change (see, e.g., Leroy et al. 2016), so many of the same considerations described above will apply. We intend to return to this topic using high-resolution $\mathrm{CO}$ observations of our targets.

\subsection{Caveats and Next Steps}

Resolved maps of density-sensitive line ratios across galaxies are new. We have focused on scaling relations that relate closely to observables. Moreover, we placed particular emphasis on $\mathrm{HCN}$, the brightest high critical density millimeter-wave line, and we use our other lines to investigate the robustness of our physical interpretation. We also target a limited area and set of targets designed to highlight the contrast between galaxy disks and central starburst regions. In this section, we highlight some of the caveats and logical next steps related to these choices.

First, our survey does not yet offer a representative sample. It was designed to capture variations in $\Sigma_{\mathrm{SFR}} / \Sigma_{\text {mol }}$ rather than to span whole galaxy disks or the local galaxy population. Thus, our scaling relations should be taken as indicative rather than authoritative. In particular, we do not yet know how they would extend to the low surface density, outer parts of galactic disks. We also lack sizable samples of barred and unbarred galaxies to quantitatively estimate the impact of a strong bar. We expect the full EMPIRE sample to help with this, as well as future deeper, wide-area mapping by the GBT and ALMA.

Second, we adopt a simple translation from $\mathrm{HCN}$ and $\mathrm{CO}$ to dense and total molecular gas mass. Given the novelty of widearea $\mathrm{HCN}$ mapping, we emphasize reporting the basic scaling relations. We also checked that our other dense gas tracers yield qualitatively similar results. Moving forward, a next direction for this field is clearly to combine a large suite of lines to model the density distribution; see Leroy et al. (2017b) for a first step in this direction, following Krumholz \& Thompson (2007) and Narayanan et al. (2008). Just as important, higher $J$ transitions are needed to understand the impact of the excitation effect. Optically isotopologue observations are needed to constrain the optical depth of the dense gas tracers, which directly impacts their $n_{\text {eff }}$ (see Jiménez-Donaire et al. 2017a). Extragalactic observations lack an external constraint on the dense gas mass (such as dust observations can provide in local clouds). As a result, wide-area Milky Way mapping of diverse environments (Pety et al. 2017) has a crucial role to play in informing the translation from line ratios to the density distribution.

We also adopt a simplified treatment of $\alpha_{\mathrm{CO}}$ in order to remain close to observables. Based on Sandstrom et al. (2013), who studied some of our targets, we know that this represents an oversimplification. $\alpha_{\mathrm{CO}}$ often drops toward the bright centers of barred galaxies. However, as discussed by Usero et al. (2015), implementing $\alpha_{\mathrm{CO}}$ corrections with no knowledge of $\alpha_{\mathrm{HCN}}$ variations may obscure the basic scaling relations. Still, our targets have also been mapped by ALMA with the explicit goal of deriving $\alpha_{\mathrm{CO}}$ from resolved multiline modeling and dust comparison. We expect to revisit this topic in detail in future work.

Other physical parameter estimates may also induce uncertainty. We adopt a combination of $\mathrm{H} \alpha$ and $24 \mu \mathrm{m}$ to trace star formation. Much of the starburst literature (Gao \& Solomon 2004; García-Burillo et al. 2012) has focused on bolometric infrared emission as a star formation tracer, motivating us to explore the impact of adopting other SFR tracers (e.g., IR tracer) in appendix. While there are subtle differences in using TIR, UV and $24 \mu \mathrm{m}$, or $\mathrm{H} \alpha$ and $24 \mu \mathrm{m}$, none of them appear to change our core results.

Finally, we make frequent reference to the structure of turbulent clouds, discussing the relationship between mean density, dynamical state (i.e., virial parameter), and the density distribution probed by our spectroscopy. Our galaxies are also targets of high-resolution $\left(\theta \sim 1^{\prime \prime}\right)$ ALMA CO (2-1) imaging ("PHANGS-ALMA"; A. K. Leroy et al. 2018, in preparation). A next main direction for these kinds of observations will be to directly compare the turbulent structure of clouds at $\sim 50-100$ pc scales from high-resolution imaging to the density distribution inferred from spectroscopy. The same data set will allow detailed comparison of the structure of the nuclear region and gas flow along the bars to the density distribution.

\section{Summary}

Our key findings are as follows:

1. We find strong central enhancements in all molecular lines, including tracers of dense gas and tracers of star formation in the central regions of our three targets with strong bars (see Figures 1-5). These inner regions show high ratios of dense gas tracers to $\mathrm{CO}$ emission (e.g., $I_{\mathrm{HCN}} / I_{\mathrm{CO}}$ ), but also signatures of lower $\Sigma_{\mathrm{SFR}} / I_{\mathrm{HCN}}$. These findings closely resemble those for our own Galaxy (e.g., Longmore et al. 2013; Kruijssen et al. 2014), where bar-induced streaming motions transport gas to the Galactic center, creating a wealth of dense gas, but that dense gas does not appear particularly good at forming stars. This appears to be a general phenomenon in strongly barred galaxies (see Section 3.1).

2. In contrast to some current theories, the apparent efficiency of star formation in molecular gas $\left(\Sigma_{\mathrm{SFR}} / I_{\mathrm{CO}}\right)$ is only weakly predicted by the apparent dense gas fraction $\left(I_{\mathrm{HCN}} / I_{\mathrm{CO}}\right)$. Our observations do fall on the previously observed TIR-HCN scaling, but real physical scatter in $\Sigma_{\mathrm{SFR}} / I_{\mathrm{HCN}}$ in our sample and the current literature renders the relationship weaker than is sometimes claimed. We find a Spearman rank correlation coefficient of $\sim 0.13$ relating the two quantities and a scatter of a factor of $\sim 2$ in SFR-to$\mathrm{HCN}$ ratio. How this result extends to the wider, fainter regions of whole galaxy disks remains to be seen (Section 3.2.1).

3. $I_{\mathrm{HCO}+} / I_{\mathrm{CO}}$ correlates well with $I_{\mathrm{HCN}} / I_{\mathrm{CO}}$ with a slightly sublinear slope, while ${ }^{13} \mathrm{CO}$ shows a flatter correlation. These are the correlations expected for a density distribution with a mean above the effective density for ${ }^{13} \mathrm{CO}$ emission but below that of $\mathrm{HCO}^{+}$and $\mathrm{HCN}$. These results give some confidence that $I_{\mathrm{HCN}} / I_{\mathrm{CO}}$ indeed traces the density distribution, but the suite of tracers available 
Table 9

Conversion of 70 and $24 \mu \mathrm{m}$ to TIR

\begin{tabular}{llc}
\hline \hline Galaxy & \multicolumn{1}{c}{$\log _{10}\left(\mathrm{TIR}_{24}\right)$} & $\log _{10}\left(\mathrm{TIR}_{70}\right)$ \\
\hline NGC 3351 & $\log _{10}\left(I_{24}\right) \times 0.853+5.772$ & $\log _{10}\left(I_{70}\right) \times 0.852+5.452$ \\
NGC 3627 & $\log _{10}\left(I_{24}\right) \times 0.984+1.504$ & $\log _{10}\left(I_{70}\right) \times 0.885+4.285$ \\
NGC 4254 & $\log _{10}\left(I_{24}\right) \times 0.948+2.779$ & $\log _{10}\left(I_{70}\right) \times 0.893+4.081$ \\
NGC 4321 & $\log _{10}\left(I_{24}\right) \times 0.923+3.550$ & $\log _{10}\left(I_{70}\right) \times 0.862+5.092$ \\
NGC 5194 $(r<2.5 \mathrm{kpc})$ & $\log _{10}\left(I_{24}\right) \times 0.919+3.803$ & $\log _{10}\left(1.1807 \times 10^{6}\left(1.93+0.01 r+0.28 r^{2}-0.048 r^{3}\right) I_{70} \times L_{\odot}[\right.$ Watts] $)$ \\
NGC $5194(r \geqslant 2.5 \mathrm{kpc})$ & $\log _{10}\left(I_{24}\right) \times 0.919+3.803$ & $\log _{10}\left(3.495 I_{70} \times L_{\odot}\left[\right.\right.$ Watts] $\left.\times 10^{6}\right)$ \\
\hline
\end{tabular}

Note.

${ }^{\mathrm{a}}$ Both $\mathrm{TIR}_{24}$ and $\mathrm{TIR}_{70}$ should have units of $\mathrm{W} \mathrm{kpc}^{-2}$.

to extragalactic studies remains limited, and much more work needs to be done to understand the translation of observed line ratios to gas density distributions (see Section 3.3).

4. $\Sigma_{\mathrm{SFR}} / I_{\mathrm{HCN}}$, an observational tracer of the star formation efficiency of dense gas, anticorrelates with the apparent surface densities of stars, traced by $3.6 \mu \mathrm{m}$ emission, and molecular gas, traced by $I_{\mathrm{CO}}$ (see Figure 10). Indeed, these correlations are stronger than that between $I_{\mathrm{HCN}} / I_{\mathrm{CO}}$ and $\Sigma_{\mathrm{SFR}} / I_{\mathrm{CO}}$. This provides additional support for a context-dependent role for density in star formation, building on the results of García-Burillo et al. (2012), Usero et al. (2015), and Bigiel et al. (2016), as well as the Milky Way results mentioned above (Sections 3.4 and 4.1).

5. On the other hand, $I_{\mathrm{HCN}} / I_{\mathrm{CO}}$, an observational tracer of the dense gas fraction, positively correlates with the apparent surface densities of stars, traced by $3.6 \mu \mathrm{m}$ emission, and molecular gas, traced by $I_{\mathrm{CO}}$ (see Figure 8). Thus, in the high surface density, deep potential well regions of galaxies, the dense gas fraction increases. There is a higher fraction of dense gas where there is more gas, and the density distribution appears to be tied to the gravitational potential well in which it sits (see Section 3.5). This result shows that trends seen among galaxies or in individual galaxies (e.g., Gao \& Solomon 2004; García-Burillo et al. 2012; Chen et al. 2015; Usero et al. 2015; Bigiel et al. 2016) also hold in resolved profiles of a sample of galaxies.

6. Both of these correlations can be re-expressed in terms of the dynamical equilibrium pressure, $P_{\mathrm{DE}}$, that must be maintained, on average, to support the weight of gas in a part of the galaxy (e.g., Elmegreen 1989; Ostriker et al. 2010; Kim et al. 2011). $I_{\mathrm{HCN}} / I_{\mathrm{CO}}$ correlates with $P_{\mathrm{DE}}$, supporting with a large amount of data the suggestion by Helfer \& Blitz (1997) of a link between ISM pressure and dense gas fraction. $\Sigma_{\mathrm{SFR}} / I_{\mathrm{HCN}}$ anticorrelates with $P_{\mathrm{DE}}$, suggesting a picture in which the mean internal pressure of a cloud reflects its environment, but only the high-density tail of the density distribution of the density distribution directly participates in star formation.

This paper makes use of the following ALMA data: ADS/JAO. ALMA\#2013.1.00634.S and ADS/JAO.ALMA\#2011.0.00004. SV. ALMA is a partnership of ESO (representing its member states), NSF (USA), and NINS (Japan), together with NRC (Canada), NSC and ASIAA (Taiwan), and KASI (Republic of Korea), in cooperation with the Republic of Chile. The Joint
ALMA Observatory is operated by ESO, AUI/NRAO, and NAOJ. The National Radio Astronomy Observatory is a facility of the National Science Foundation operated under cooperative agreement by Associated Universities, Inc. This work is partially based on observations carried out with the IRAM IRAM $30 \mathrm{~m}$ telescope. IRAM is supported by INSU/CNRS (France), MPG (Germany), and IGN (Spain). The work of M.G. and A.K.L. is partially supported by the National Science Foundation under grant nos. 1615105, 1615109, and 1653300. E.S. acknowledges financial support to the DAGAL network from the People Programme (Marie Curie Actions) of the European Union's Seventh Framework Programme FP7/2007-2013/under REA grant agreement no. PITN-GA-2011-289313. A.H. acknowledges support from the Centre National d'Etudes Spatiales (CNES). A.U. acknowledges support from Spanish MINECO grants ESP2015-68964 and AYA2016-79006. E.R. is supported by a Discovery Grant from NSERC Canada. F.B. acknowledges funding from the European Unions Horizon 2020 research and innovation programme (grant agreement no. 726384-EMPIRE) This paper made use of the NASA Astrophysics Data Abstract service, AstroPy, the IDL Astronomy User's Library, CASA, and the NASA Extragalactic Database. M.G. and A.K.L. gratefully acknowledge helpful discussions with Todd Thompson and the OSU galaxy and ISM discussion groups. Diane Cormier is supported by the European Union's Horizon 2020 research and innovation programme under the Marie Skłodowska-Curie grant agreement no. 702622. M.J.J.-D. and F.B. acknowledge support from DFG grant BI 1546/ 1-1. E.O. is supported by grant AST-7173949 from the National Science Foundation.

\section{Appendix A \\ $70 \mu \mathrm{m}$ Emission, Total Infrared Emission, and SFR Estimation in Our Targets}

As discussed in Section 2.5.2, we are interested in estimating the TIR luminosity surface density, $\Sigma_{\mathrm{TIR}}$, for each ring. The bolometric infrared emission has been a standard measure of recent star formation in the HCN and "dense gas" literature, and it offers an alternative to and a check on our fiducial $\mathrm{H} \alpha$ $+24 \mu \mathrm{m}$ approach.

To calculate $\Sigma_{\mathrm{TIR}}$, we use maps of $70,100,160$, and $250 \mu \mathrm{m}$ emission from Herschel and of $24 \mu \mathrm{m}$ emission from Spitzer. We used the kernels provided by Aniano et al. (2011) to convolve all of these maps to share a Gaussian 30" (FWHM) beam. At this coarse resolution, we calculate $\Sigma_{\text {TIR }}$ using all bands and following the prescription of Galametz et al. (2013).

Ideally, we would simply use all of these data to estimate $\Sigma_{\text {TIR }}$ ring by ring. Unfortunately, the 160 and $250 \mu \mathrm{m}$ data, which constrain the long-wavelength part of the IR spectral 

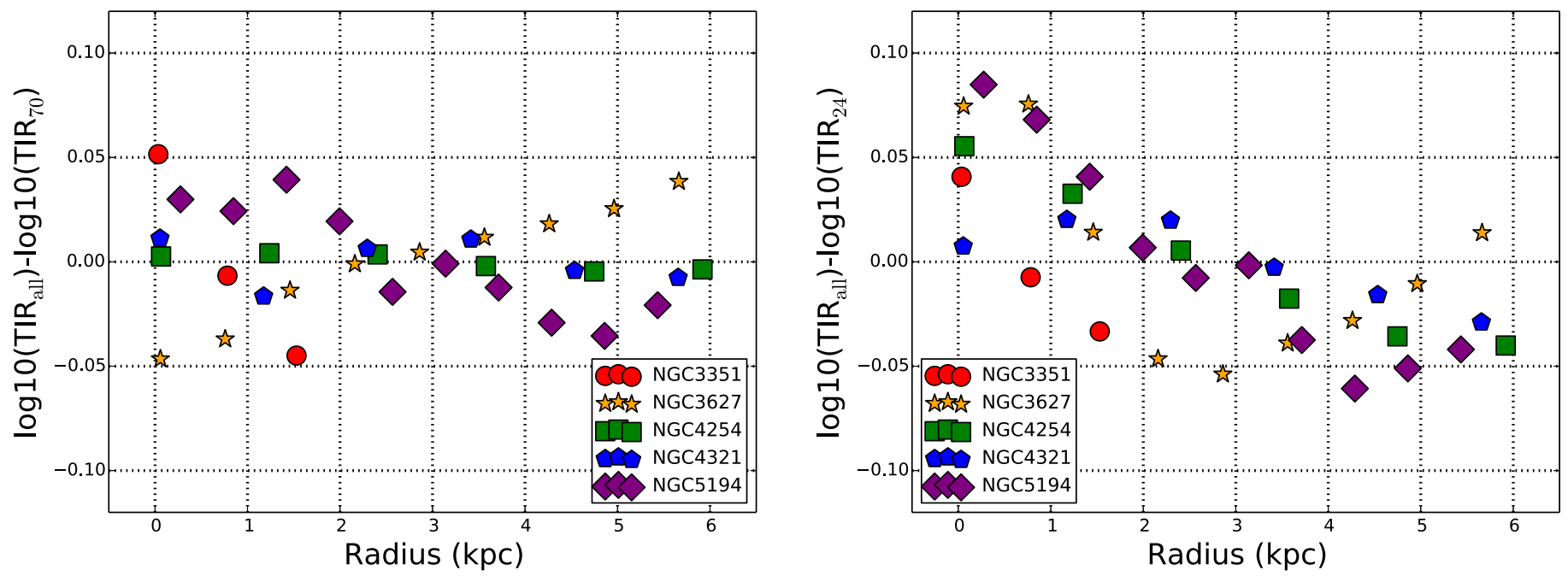

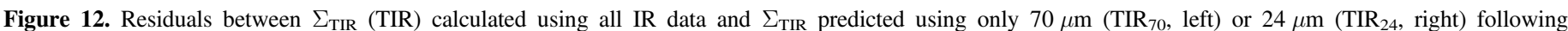

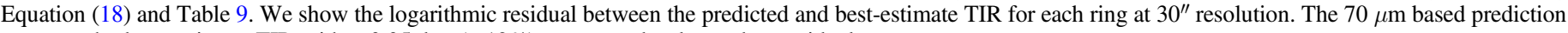
recovers the best-estimate TIR with $\sim 0.05 \operatorname{dex}(\sim 12 \%)$ scatter and only modest residuals.

energy distribution, have coarse angular resolution compared to our $8^{\prime \prime}$ working resolution. Therefore, we use Herschel PACS $70 \mu \mathrm{m}$ emission $\left(I_{70}\right)$ as a proxy for the TIR surface density $\left(\Sigma_{\mathrm{TIR}}\right)$.

To do this, we adopt an empirical approach calibrated galaxy by galaxy. For each target, we fit a relation between $\Sigma_{\text {TIR }}$ and $70 \mu \mathrm{m}$ intensity, $I_{70}$, at $30^{\prime \prime}$ resolution and assume that that relation holds at the higher $8^{\prime \prime}$ resolution of the data. The relation has the form

$$
\log _{10} \Sigma_{\mathrm{TIR}}=a \log _{10} I_{70}+b .
$$

Here $I_{70}$ is the surface brightness at $70 \mu \mathrm{m}, \Sigma_{\mathrm{TIR}}$ is TIR luminosity surface density, and $a$ and $b$ are the best-fit constants. Galametz et al. (2013) already calculated galaxyspecific $a$ and $b$ for all four of our ALMA targets. We modify their values slightly based on our own fits to the data for the specific fields of view that we study and choice of resolution. We do not consider these prescriptions as corrections to Galametz et al. (2013), only more appropriate for the precise data that we study. Table 9 reports our adopted conversion for each galaxy. For M51, we adopt the prescription by Leroy et al. (2017a), who carried out a similar exercise in that galaxy in an overlapping region.

We calculate a similar conversion using $24 \mu \mathrm{m}$ emission to

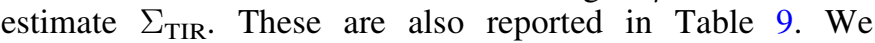
consider the $70 \mu \mathrm{m}$ based $\Sigma_{\mathrm{TIR}}$ estimates more reliable than the $24 \mu \mathrm{m}$ based ones, which agrees with results from Calzetti et al. (2010) and Galametz et al. (2013). The $70 \mu \mathrm{m}$ band lies nearer to the peak of the IR SED, and as a result our $70 \mu \mathrm{m}$ based fits recover $\Sigma_{\mathrm{TIR}}$ at $30^{\prime \prime}$ resolution with less residual scatter than the $24 \mu \mathrm{m}$ based fits. We plot the residuals about the best-fit relation in Figure 12. The figure shows the difference between the TIR predicted using a single band (70 or $24 \mu \mathrm{m}$ ) with Table 9 and the calculation using all bands. The $70 \mu \mathrm{m}$ based approach predicts $\Sigma_{\text {TIR }}$ with better than $\pm 0.05 \operatorname{dex}(\sim 12 \%)$ accuracy and only weak residual trends. The $24 \mu \mathrm{m}$ based approach (right panel) shows larger residuals and systematic trends - a power law is an inadequate description of the $24 \mu \mathrm{m}$-to-TIR relationship. Coupled with the cleaner and more centrally peaked PSF, this leads us to prefer a $70 \mu \mathrm{m}$ based $\Sigma_{\mathrm{TIR}}$ estimate at $8^{\prime \prime}$ resolution.

We present these estimates in the table described in Appendix $\mathrm{C}$ and recommend them for those interested in using the bolometric luminosity of young stars to capture recent star formation. They provide a useful check when compared with the recombination-line-based estimates used in the main paper.

Note that these bolometric IR estimates carry the usual uncertainties related to semi-resolved SFR estimates. The star formation history over the past $\sim 100$ Myr may vary from place to place, and some fraction of the emitted starlight may not be reprocessed by dust (though see below). Dust properties may also vary, perhaps driving some of the observed scatter in the $70 \mu \mathrm{m}$-to-TIR ratio. Calzetti (2013) and Kennicutt \& Evans (2012) review key systematics for both this approach and our fiducial $\mathrm{H} \alpha+24 \mu \mathrm{m}$ tracer.

Also note that Calzetti et al. (2010) have calibrated the monochromatic $70 \mu \mathrm{m}$ as an SFR indicator for whole galaxies. The difference in scales between that study and this one will likely introduce a difference in the mean age of the population sampled. As a result, we do not expect their prescription to apply exactly to our case. Li et al. (2013) observe exactly such a scale dependence in a study using $\operatorname{Br} \gamma$ to calibrate $70 \mu \mathrm{m}$ emission as an SFR tracer at several scales in two galaxies. We refer readers interested in more details on the performance of $70 \mu \mathrm{m}$ based tracers of recent star formation to those papers and the reviews listed above.

Dust heating by an older stellar population may also contaminate our IR-based SFR estimates, though we expect this to be subdominant in our sample. Studying $24 \mu \mathrm{m}$ emission from our targets, Leroy et al. (2012) estimated that 7\% (NGC 3351), 12\% (NGC 3627), 16\% (NGC 4254), 23\% (NGC 4321), and $35 \%$ (NGC 5194) of the $24 \mu \mathrm{m}$ light within $r_{\text {gal }}<4 \mathrm{kpc}$ arises from dust heated by an older stellar population. Though these estimates have substantial uncertainty and apply to $24 \mu \mathrm{m}$ rather than $70 \mu \mathrm{m}$, they give an idea of the likely magnitude of a "cirrus" correction in these targets (see also Liu et al. 2011). 


\section{Appendix B \\ Different Star Formation Tracers}

In the main text, we use a combination of $24 \mu \mathrm{m}$ and $\mathrm{H} \alpha$ emission to estimate $\Sigma_{\text {SFR. }}$. Here, we explore the consequences of adopting other SFR tracers. We estimate $\Sigma_{\text {SFR }}$ based on the 70 and $24 \mu \mathrm{m}$ based $\Sigma_{\text {TIR }}$ described in the previous section, $\mathrm{H} \alpha$ alone, and far-UV (FUV) emission. We compare these to our fiducial $\mathrm{H} \alpha+24 \mu \mathrm{m}$ estimates and look at the relative contribution of different terms. We also show that our basic conclusions are robust to the choice of SFR tracer.

For this comparison, we calculate $\Sigma_{\mathrm{SFR}}$ from the following:

1. $\Sigma_{\mathrm{TIR}}$ estimated from $I_{70}$ and $I_{24}$ (separately) following Table 9 and using Equation (6).

2. FUV only using the coefficient quoted in Leroy et al. (2012), consistent with the one in Kennicutt \& Evans (2012).

3. A combination of FUV and $24 \mu \mathrm{m}$ emission following Leroy et al. (2012).

4. $\mathrm{H} \alpha$ only using that part of Equation (5).
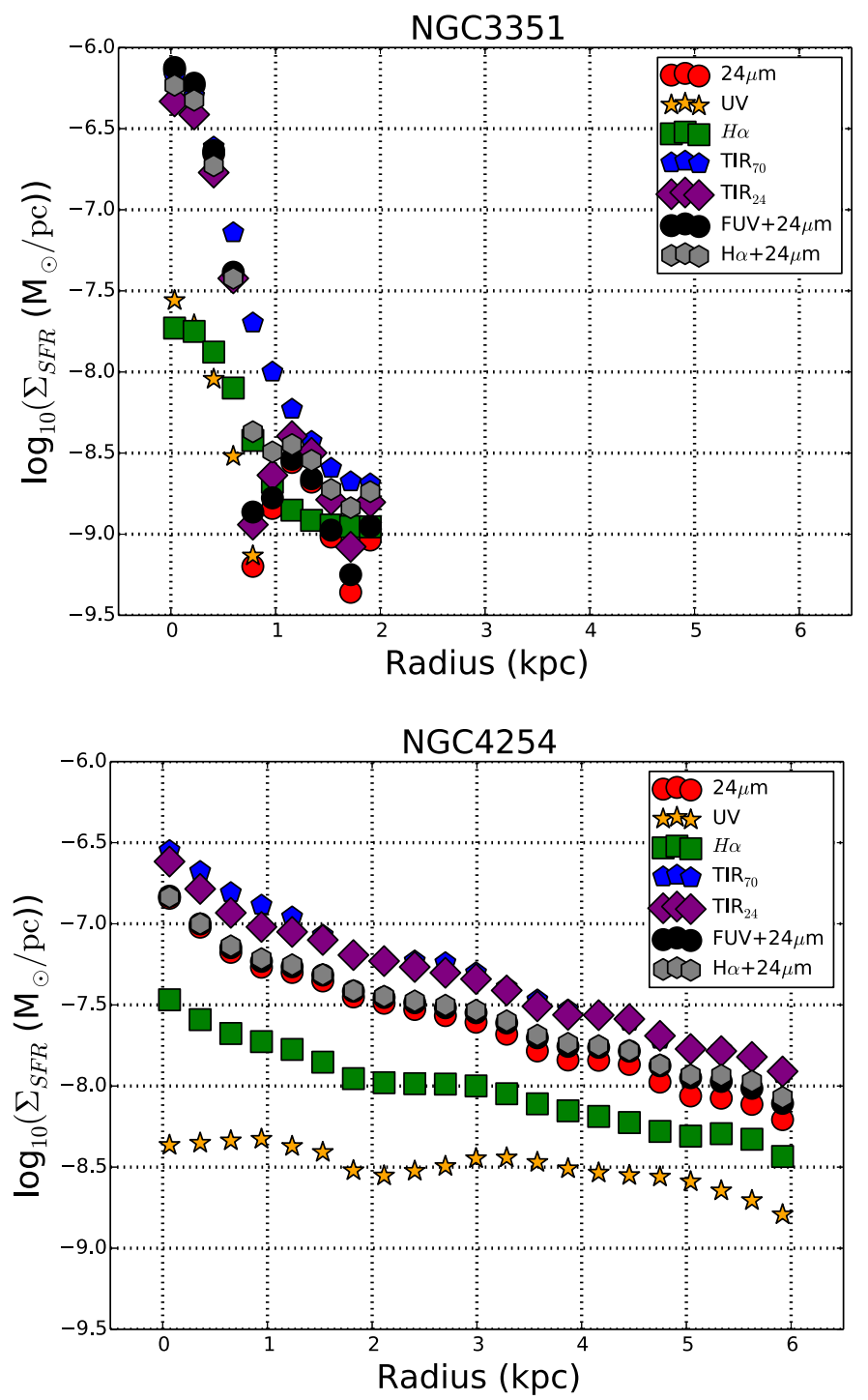

5. $24 \mu \mathrm{m}$ only using that part of Equation (5), which is our estimate for extinguished $\mathrm{H} \alpha$ emission.

Figure 13 shows the radial profiles of $\Sigma_{\text {SFR }}$ for each of these estimates. In all cases, the IR-based tracers imply higher $\Sigma_{\text {SFR }}$ than either $\mathrm{H} \alpha$ or FUV alone. In fact, the figure shows that over the area of interest in all of our targets, the contribution from the unobscured FUV term (yellow stars) to any $\Sigma_{\mathrm{SFR}}$ estimate is negligible. Estimates of $\Sigma_{\text {SFR }}$ using only $\mathrm{H} \alpha$ (green squares) are also lower everywhere than any estimate involving IR emission. This could be expected given that we focus on gasrich inner regions of actively star-forming galaxies. As a result, over most of our target area the "hybrid" tracers combining $24 \mu \mathrm{m}$ emission and FUV or $\mathrm{H} \alpha$ yield results that are mostly indistinguishable from using only the $24 \mu \mathrm{m}$ part of the coefficient.

As a result of this bright IR emission compared to other tracers, Figure 13 also supports the utility of our alternative, pure infrared estimator. Though the caveats listed in the
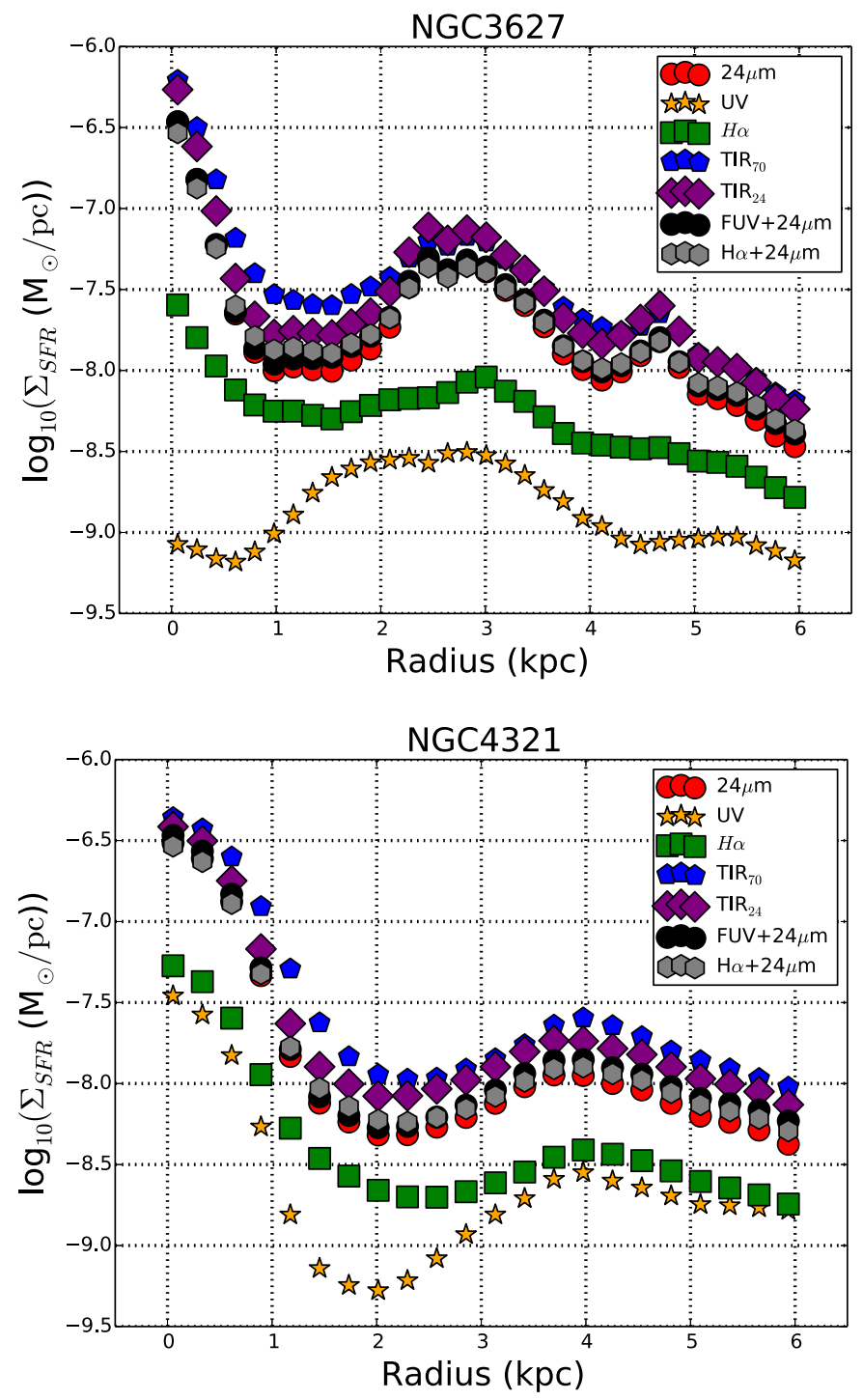

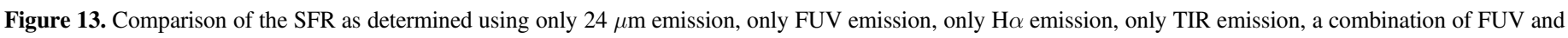

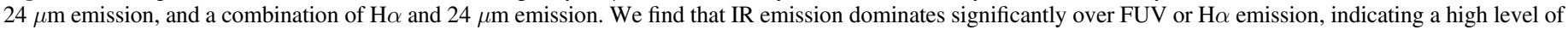
extinction. 
Table 10

$\Sigma_{\mathrm{SFR}} / \Sigma_{\mathrm{mol}}$ vs. $\Sigma_{\text {dense }} / \Sigma_{\mathrm{mol}}$

\begin{tabular}{lc}
\hline \hline SFR Tracer & Rank Corr. \\
\hline $\mathrm{TIR}_{70}$ & $0.28(0.000)$ \\
$\mathrm{TIR}_{24}$ & $0.16(0.002)$ \\
$\mathrm{FUV}+24 \mu \mathrm{m}$ & $0.13(0.003)$ \\
$\mathrm{H} \alpha+24 \mu \mathrm{m}$ & $0.13(0.003)$ \\
\hline
\end{tabular}

Note. Rank correlation quotes with $p$ value in parentheses. Each data point represents the result using all five data profiles, including NGC 5194 (Bigiel et al. 2016)

previous section apply, the plots suggest that across our sample most of the light from young stars is reprocessed by dust. We still cannot rule out important "cirrus" contributions to the IR emission from these regions (see the estimates noted in the previous section). But we do see that our targets are high $\Sigma_{\mathrm{SFR}}$ in all tracers, giving us some confidence that the cirrus contribution is likely to be subdominant.

One of our main results is that only the measured $\mathrm{HCN} / \mathrm{CO}$ ratio in a ring is not a perfect, or even necessarily very good, predictor of the ratio $\Sigma_{\mathrm{SFR}} / \mathrm{CO}$. We test whether that conclusion changes if we substitute a different SFR tracer for that which we use in the main paper. Equations (19)-(22) and Table 10 compare the effects of using the three different types of SFR tracers on our main result. We see that our result, that the dense gas fraction is not a good tracer of the molecular gas star formation efficiency, does not depend on our choice of SFR tracer. For this comparison, we convert $\mathrm{HCN}$ and $\mathrm{CO}$ emission into dense gas and molecular gas surface densities, respectively, in order to compare physical quantities. For a more detailed description of these conversions, see Section 2.3. Here, we make no correction for IR cirrus. This is an area for future improvement (but for a comparison that does check the effect of IR cirrus estimates on similar results, see Usero et al. 2015).

These four SFR calculations yield the following fitted relations:

$$
\begin{gathered}
\log _{10} \frac{\Sigma_{\text {SFR,TIR,70 }}}{\Sigma_{\text {mol }}}=-7.89+0.85 \log _{10} \frac{\Sigma_{\text {dense }}}{\Sigma_{\text {mol }}} \\
\log _{10} \frac{\Sigma_{\text {SFR,TIR,24 }}}{\Sigma_{\text {mol }}}=-7.95+0.88 \log _{10} \frac{\Sigma_{\text {dense }}}{\Sigma_{\text {mol }}} \\
\log _{10} \frac{\Sigma_{\text {SFR,UV }+24}}{\Sigma_{\text {mol }}}=-8.02+0.94 \log _{10} \frac{\Sigma_{\text {dense }}}{\Sigma_{\text {mol }}} \\
\log _{10} \frac{\Sigma_{\text {SFR }, \mathrm{H} \alpha+24}}{\Sigma_{\text {mol }}}=-8.05+0.92 \log _{10} \frac{\Sigma_{\text {dense }}}{\Sigma_{\text {mol }}} .
\end{gathered}
$$

The slopes and intercepts of all three relationships are not identical, indicating that the choice of SFR tracer does have some impact on our results. However, no matter the choice of tracer, the rank correlation coefficient relating $\Sigma_{\mathrm{SFR}} / \Sigma_{\mathrm{mol}}$ to $\Sigma_{\text {dense }} / \Sigma_{\text {mol }}$ is low when we consider all galaxies together. These values appear in Table 10. None of these SFR tracers yield strong correlations with dense gas fraction, and neither the fit nor the correlation changes significantly with SFR tracer.

Usero et al. (2015) explore the issue of different SFR tracers, using TIR, $\mathrm{H} \alpha$, and $24 \mu \mathrm{m}$ data, as well as a combination of $\mathrm{H} \alpha$ and $24 \mu \mathrm{m}$. They find that all SFR tracers yield similar trends, albeit with different levels of intrinsic scatter. Leroy et al. (2012) also find good agreement between the results using different SFR tracers. They use $\mathrm{H} \alpha$ with one magnitude of extinction, FUV and $24 \mu \mathrm{m}, \mathrm{H} \alpha$ and $24 \mu \mathrm{m}$, and $\mathrm{H} \alpha$ and $24 \mu \mathrm{m}$ with a correction for cirrus.

Our analysis indicates that while there is some variation, the choice of SFR tracer does not change our qualitative results. Quantitatively, a more rigorous examination of what SFR tracers are valid in these different galactic environments is needed. Such an examination is beyond the scope of this paper.

\section{Appendix C Radial Profile Data Table}

We provide the radial profiles used in this paper as a machine-readable table. In this table, each row reports our measurements for one radial ring in one galaxy. The contents of each row are as follows:

1. The NGC number of the galaxy, and the inner radius of the ring, in $\mathrm{kpc}$.

2. The average intensity and rms uncertainty on the average intensity for the $\mathrm{CO}(1-0), \mathrm{HCN}(1-0), \mathrm{CS}(2-1), \mathrm{HCO}^{+}$ $(1-0),{ }^{13} \mathrm{CO}(1-0)$, and $\mathrm{C}^{18} \mathrm{O}(1-0)$ emission lines.

3. The average intensity and rms uncertainty for 3.6, 24, $70 \mu \mathrm{m}, \mathrm{FUV}$, and $\mathrm{H} \alpha$ emission.

4. Quantities derived from the intensities above and the assumptions described in Section 2: dynamical equilibrium pressure, stellar surface density, TIR luminosity surface density, total (H I+molecular), molecular, and dense gas surface density.

5. Estimates of the SFR surface density constructed using several approaches, as well as individual terms in the hybrid estimators discussed in Section 2 and Appendix B.

\section{ORCID iDs}

Molly J. Gallagher (iD https://orcid.org/0000-0001-5285-5930

Adam K. Leroy (ib https://orcid.org/0000-0002-2545-1700

Frank Bigiel (i) https://orcid.org/0000-0003-0166-9745

Diane Cormier (1) https://orcid.org/0000-0002-1046-2685

María J. Jiménez-Donaire (i) https://orcid.org/0000-00029165-8080

Eve Ostriker (iD https://orcid.org/0000-0002-0509-9113

Antonio Usero (ib https://orcid.org/0000-0003-1242-505X

Alberto D. Bolatto (ib https://orcid.org/0000-0002-5480-5686

Santiago García-Burillo (1) https://orcid.org/0000-00030444-6897

Annie Hughes (iD https://orcid.org/0000-0002-9181-1161

Amanda A. Kepley (iD https://orcid.org/0000-0002-3227-4917

Mark Krumholz (i) https://orcid.org/0000-0003-3893-854X

Sharon E. Meidt (ib https://orcid.org/0000-0002-6118-4048

David S. Meier (iD https://orcid.org/0000-0001-9436-9471

Eric J. Murphy (1D https://orcid.org/0000-0001-7089-7325

Jérôme Pety (i) https://orcid.org/0000-0003-3061-6546

Erik Rosolowsky (i) https://orcid.org/0000-0002-5204-2259

Eva Schinnerer (i) https://orcid.org/0000-0002-3933-7677

Fabian Walter (iD https://orcid.org/0000-0003-4793-7880

\section{References}

André, P., Di Francesco, J., Ward-Thompson, D., et al. 2014, in Protostars and Planets VI, ed. H. Beuther et al. (Tucson, AZ: Univ. Arizona Press), 27 Aniano, G., Draine, B. T., Gordon, K. D., \& Sandstrom, K. 2011, PASP, 123,1218

Beuther, H., Bihr, S., Rugel, M., et al. 2016, A\&A, 595, A32

Bigiel, F., Leroy, A. K., Blitz, L., et al. 2015, ApJ, 815, 103 
Bigiel, F., Leroy, A. K., Jiménez-Donaire, M. J., et al. 2016, ApJL, 822, L26 Blitz, L., \& Rosolowsky, E. 2006, ApJ, 650, 933

Boissier, S., Prantzos, N., Boselli, A., \& Gavazzi, G. 2003, MNRAS, 346, 1215

Bolatto, A. D., Wolfire, M., \& Leroy, A. K. 2013, ARA\&A, 51, 207

Bolatto, A. D., Wong, T., Utomo, D., et al. 2017, ApJ, 846, 159

Braine, J., \& Combes, F. 1992, A\&A, 264, 433

Brouillet, N., Muller, S., Herpin, F., Braine, J., \& Jacq, T. 2005, A\&A, 429, 153

Caldú-Primo, A., Schruba, A., Walter, F., et al. 2013, AJ, 146, 150

Calzetti, D. 2013, in Secular Evolution of Galaxies, ed. J. Falcón-Barroso \& J. H. Knapen (Cambridge: Cambridge Univ. Press), 419

Calzetti, D., Kennicutt, R. C., Engelbracht, C. W., et al. 2007, ApJ, 666, 870

Calzetti, D., Wu, S.-Y., Hong, S., et al. 2010, ApJ, 714, 1256

Chen, H., Gao, Y., Braine, J., \& Gu, Q. 2015, ApJ, 810, 140

Colombo, D., Meidt, S. E., Schinnerer, E., et al. 2014, ApJ, 784, 4

Comerón, S., Elmegreen, B. G., Salo, H., et al. 2012, ApJ, 759, 98

Comerón, S., Elmegreen, B. G., Salo, H., et al. 2014, A\&A, 571, A58

Cormier, D., Bigiel, F., Jiménez-Donaire, M. J., et al. 2018, MNRAS, 475, 3909

de Blok, W. J. G., Walter, F., Brinks, E., et al. 2008, AJ, 136, 2648

de Vaucouleurs, G., de Vaucouleurs, A., Corwin, H. G., Jr., et al. 1991, Third

Reference Catalogue of Bright Galaxies, Vol. I, II, III (New York: SpringerVerlag)

Dicaire, I., Carignan, C., Amram, P., et al. 2008, MNRAS, 385, 553

Downes, D., Reynaud, D., Solomon, P. M., \& Radford, S. J. E. 1996, ApJ, 461, 186

Elmegreen, B. G. 1989, ApJ, 338, 178

Evans, N. J., II, Heiderman, A., \& Vutisalchavakul, N. 2014, ApJ, 782, 114

Federrath, C., \& Klessen, R. S. 2013, ApJ, 763, 51

Galametz, M., Kennicutt, R. C., Calzetti, D., et al. 2013, MNRAS, 431, 1956

Gao, Y., \& Solomon, P. M. 2004, ApJ, 606, 271

García-Burillo, S., Usero, A., Alonso-Herrero, A., et al. 2012, A\&A, 539, A8

Graciá-Carpio, J., García-Burillo, S., Planesas, P., \& Colina, L. 2006, ApJL, 640, L135

Graciá-Carpio, J., García-Burillo, S., Planesas, P., Fuente, A., \& Usero, A. 2008, A\&A, 479, 703

Heiderman, A., Evans, N. J., II, Allen, L. E., Huard, T., \& Heyer, M. 2010, ApJ, 723, 1019

Helfer, T. T., \& Blitz, L. 1997, ApJ, 478, 162

Helfer, T. T., Thornley, M. D., Regan, M. W., et al. 2003, ApJS, 145, 259

Heyer, M., \& Dame, T. M. 2015, ARA\&A, 53, 583

Hughes, A., Meidt, S. E., Colombo, D., et al. 2013, ApJ, 779, 46

Imanishi, M., Nakanishi, K., \& Kohno, K. 2006, AJ, 131, 2888

Jiménez-Donaire, M. J., Bigiel, F., Leroy, A. K., et al. 2017a, MNRAS, 466, 49

Jiménez-Donaire, M. J., Cormier, D., Bigiel, F., et al. 2017b, ApJL, 836, L29

Kainulainen, J., Beuther, H., Henning, T., \& Plume, R. 2009, A\&A, 508, L35

Kauffmann, J., Pillai, T., \& Zhang, Q. 2013, ApJL, 765, L35

Kauffmann, J., Pillai, T., Zhang, Q., et al. 2017, A\&A, 603, A90

Kawamura, A., Mizuno, Y., Minamidani, T., et al. 2009, ApJS, 184, 1

Kennicutt, R. C., Calzetti, D., Aniano, G., et al. 2011, PASP, 123, 1347

Kennicutt, R. C., \& Evans, N. J. 2012, ARA\&A, 50, 531

Kennicutt, R. C., Jr., Armus, L., Bendo, G., et al. 2003, PASP, 115, 928

Kennicutt, R. C., Jr., Calzetti, D., Walter, F., et al. 2007, ApJ, 671, 333

Kepley, A. A., Leroy, A. K., Frayer, D., et al. 2014, ApJL, 780, L13

Kim, C.-G., Kim, W.-T., \& Ostriker, E. C. 2011, ApJ, 743, 25

Kim, C.-G., \& Ostriker, E. C. 2015, ApJ, 815, 67

Kim, C.-G., \& Ostriker, E. C. 2017, ApJ, 846, 133

Kim, C.-G., Ostriker, E. C., \& Kim, W.-T. 2013, ApJ, 776, 1

Könyves, V., André, P., Men'shchikov, A., et al. 2015, A\&A, 584, A91

Kregel, M., van der Kruit, P. C., \& de Grijs, R. 2002, MNRAS, 334, 646

Kruijssen, J. M. D., Longmore, S. N., Elmegreen, B. G., et al. 2014, MNRAS, 440, 3370

Krumholz, M. R., \& Kruijssen, J. M. D. 2015, MNRAS, 453, 739

Krumholz, M. R., \& McKee, C. F. 2005, ApJ, 630, 250

Krumholz, M. R., \& Tan, J. C. 2007, ApJ, 654, 304
Krumholz, M. R., \& Thompson, T. A. 2007, ApJ, 669, 289

Lada, C. J., Forbrich, J., Lombardi, M., \& Alves, J. F. 2012, ApJ, 745, 190

Lada, C. J., \& Lada, E. A. 2003, ARA\&A, 41, 57

Lada, C. J., Lombardi, M., \& Alves, J. F. 2010, ApJ, 724, 687

Lee, J. C., Gil de Paz, A., Tremonti, C., et al. 2009, ApJ, 706, 599

Leroy, A. K., Bigiel, F., de Blok, W. J. G., et al. 2012, AJ, 144, 3

Leroy, A. K., Hughes, A., Schruba, A., et al. 2016, ApJ, 831, 16

Leroy, A. K., Lee, C., Schruba, A., et al. 2013a, ApJL, 769, L12

Leroy, A. K., Schinnerer, E., Hughes, A., et al. 2017a, ApJ, 846, 71

Leroy, A. K., Usero, A., Schruba, A., et al. 2017b, ApJ, 835, 217

Leroy, A. K., Walter, F., Bigiel, F., et al. 2009, AJ, 137, 4670

Leroy, A. K., Walter, F., Brinks, E., et al. 2008, AJ, 136, 2782

Leroy, A. K., Walter, F., Martini, P., et al. 2015, ApJ, 814, 83

Leroy, A. K., Walter, F., Sandstrom, K., et al. 2013b, AJ, 146, 19

Li, Y., Crocker, A. F., Calzetti, D., et al. 2013, ApJ, 768, 180

Liu, G., Koda, J., Calzetti, D., Fukuhara, M., \& Momose, R. 2011, ApJ, 735,63

Longmore, S. N., Bally, J., Testi, L., et al. 2013, MNRAS, 429, 987

Martín, S., Mauersberger, R., Martín-Pintado, J., Henkel, C., \& García-Burillo, S. 2006, ApJS, 164, 450

McMullin, J. P., Waters, B., Schiebel, D., Young, W., \& Golap, K. 2007, in ASP Conf. Ser. 376, Astronomical Data Analysis Software and Systems XVI, ed. R. A. Shaw, F. Hill, \& D. J. Bell (San Francisco, CA: ASP), 127 Meidt, S. E. 2016, ApJ, 818, 69

Meidt, S. E., Leroy, A. K., Rosolowsky, E., et al. 2018, ApJ, 854, 100

Meidt, S. E., Schinnerer, E., Hughes, A., et al. 2013, in IAU Symp. 292, Molecular Gas, Dust, and Star Formation in Galaxies, ed. T. Wong \& J. Ott (Cambridge: Cambridge Univ. Press), 139

Meidt, S. E., Schinnerer, E., Knapen, J. H., et al. 2012, ApJ, 744, 17

Meidt, S. E., Schinnerer, E., van de Ven, G., et al. 2014, ApJ, 788, 144

Mogotsi, K. M., de Blok, W. J. G., Caldú-Primo, A., et al. 2016, AJ, 151, 15

Muñoz-Mateos, J. C., Gil de Paz, A., Boissier, S., et al. 2009, ApJ, 701, 1965

Muñoz-Mateos, J. C., Sheth, K., Gil de Paz, A., et al. 2013, ApJ, 771, 59

Murphy, E. J., Condon, J. J., Schinnerer, E., et al. 2011, ApJ, 737, 67

Murphy, E. J., Dong, D., Leroy, A. K., et al. 2015, ApJ, 813, 118

Narayanan, D., Cox, T. J., Shirley, Y., et al. 2008, ApJ, 684, 996

Ostriker, E. C., McKee, C. F., \& Leroy, A. K. 2010, ApJ, 721, 975

Ostriker, E. C., \& Shetty, R. 2011, ApJ, 731, 41

Padoan, P., \& Nordlund, A. 2002, ApJ, 576, 870

Pety, J., Guzmán, V. V., Orkisz, J. H., et al. 2017, A\&A, 599, A98

Privon, G. C., Herrero-Illana, R., Evans, A. S., et al. 2015, ApJ, 814, 39

Querejeta, M., Meidt, S. E., Schinnerer, E., et al. 2015, ApJS, 219, 5

Querejeta, M., Meidt, S. E., Schinnerer, E., et al. 2016, A\&A, 588, A33

Rahman, N., Bolatto, A. D., Wong, T., et al. 2011, ApJ, 730, 72

Rathborne, J. M., Longmore, S. N., Jackson, J. M., et al. 2015, ApJ, 802, 125

Regan, M. W., Sheth, K., \& Vogel, S. N. 1999, ApJ, 526, 97

Regan, M. W., Thornley, M. D., Helfer, T. T., et al. 2001, ApJ, 561, 218

Rosolowsky, E., Pineda, J. E., \& Gao, Y. 2011, MNRAS, 415, 1977

Saintonge, A., Kauffmann, G., Wang, J., et al. 2011, MNRAS, 415, 61

Sanders, D. B., \& Mirabel, I. F. 1996, ARA\&A, 34, 749

Sandstrom, K. M., Leroy, A. K., Walter, F., et al. 2013, ApJ, 777, 5

Schruba, A., Leroy, A. K., Walter, F., et al. 2011, AJ, 142, 37

Schruba, A., Leroy, A. K., Walter, F., Sandstrom, K., \& Rosolowsky, E. 2010, ApJ, 722, 1699

Sheth, K., Regan, M., Hinz, J. L., et al. 2010, PASP, 122, 1397

Sheth, K., Vogel, S. N., Regan, M. W., Thornley, M. D., \& Teuben, P. J. 2005, ApJ, 632, 217

Shirley, Y. L. 2015, PASP, 127, 299

Tamburro, D., Rix, H.-W., Leroy, A. K., et al. 2009, AJ, 137, 4424

Usero, A., Leroy, A. K., Walter, F., et al. 2015, AJ, 150, 115

van der Kruit, P. C. 1988, A\&A, 192, 117

Walter, F., Brinks, E., de Blok, W. J. G., et al. 2008, AJ, 136, 2563

Wong, T., \& Blitz, L. 2002, ApJ, 569, 157

Wu, J., Evans, N. J., II, Shirley, Y. L., \& Knez, C. 2010, ApJS, 188, 313

Young, J. S., \& Scoville, N. Z. 1991, ARA\&A, 29, 581 